\title{
Health Card Retrieval for Consumer Health Search
}

\author{
An Empirical Investigation of Methods \\ Jimmy $^{1,2}$, Guido Zuccon ${ }^{1}$, Bevan Koopman ${ }^{3}$, Gianluca Demartini ${ }^{1}$ \\ ${ }^{1}$ University of Queensland, Brisbane, Australia \\ ${ }^{2}$ University of Surabaya (UBAYA), Surabaya, Indonesia \\ ${ }^{3}$ Australian E-Health Research Center, CSIRO, Brisbane, Australia \\ jimmy@uqconnect.edu.au,g.zuccon@uq.edu.au,bevan.koopman@csiro.au,g.demartini@uq.edu.au
}

\begin{abstract}
This paper investigates methods to rank health cards, a domainspecific type of entity cards, for consumer health search (CHS) queries. A key challenge in this context is which card(s) should be presented to the user. In particular, little evidence exists to determine the effectiveness of retrieval and ranking methods for health cards in CHS. CHS is a challenging domain, where users lack domain expertise and thus are often unable to formulate effective queries, and to interpret the retrieved results. In addition, unlike in other contexts, CHS presents the opportunity to exploit a number of domain specific characteristics and features.

In this paper, we focus on difficult queries with self-diagnosis intents. Our study makes the following contributions: (1) it assembles and releases the first test collection of health cards for research purposes, and (2) it empirically evaluates a large range of entity retrieval methods adapted to health cards retrieval, including features specific to health cards for learning to rank. This is the first study that thoroughly investigates methods to rank health cards.
\end{abstract}

\section{KEYWORDS}

health cards, consumer health search, entity retrieval, learning to rank

ACM Reference Format:

Jimmy ${ }^{1,2}$, Guido Zuccon ${ }^{1}$, Bevan Koopman ${ }^{3}$, Gianluca Demartini ${ }^{1} .2019$. Health Card Retrieval for Consumer Health Search: An Empirical Investigation of Methods. In The 28th ACM International Conference on Information and Knowledge Management (CIKM '19), November 3-7, 2019, Beijing, China. ACM, New York, NY, USA, 4 pages. https://doi.org/10.1145/3357384.3358128

\section{INTRODUCTION}

An entity card is an information object within a search engine results page (SERP) that encapsulates a variety of information on a particular entity $[3,7,12,15,17]$. Entity cards have been used to support user search activities in the context of a user's formulated query $[7,12]$, as well as in proactive systems where relevant entity cards are shown before any query is submitted [15]. Presenting relevant entity cards is known to increase user engagement with

Permission to make digital or hard copies of all or part of this work for personal or classroom use is granted without fee provided that copies are not made or distributed for profit or commercial advantage and that copies bear this notice and the full citation on the first page. Copyrights for components of this work owned by others than ACM must be honored. Abstracting with credit is permitted. To copy otherwise, or republish, to post on servers or to redistribute to lists, requires prior specific permission and/or a fee. Request permissions from permissions@acm.org.

CIKM '19, November 3-7, 2019, Beijing, China

() 2019 Association for Computing Machinery.

ACM ISBN 978-1-4503-6976-3/19/11 . \$15.00

https://doi.org/10.1145/3357384.3358128 the search results and reduce the number of queries issued, thus improving the overall user experience [7].

In the context of consumer health search (CHS), a health card is a specific type of entity card which presents information about a specific health concept in a coherent and easy to read form $[2,10]$. Health cards are beneficial to users in the context of self-diagnosis and health decision making [10]: they reduce the effort and workload required to complete the search task, and help less knowledgeable users to take well informed health decisions.

However, while previous work has considered whether the display of a health card allowed users to make better health decisions, and if so under which conditions. Our own previous research [11] has shown that the display of multiple health cards (up to 4) better supported health search users in specific circumstances, e.g., to make differential diagnosis, when the relevance of a single health card was uncertain [11].

Research on retrieving and ranking entity cards (or even deciding if displaying a card at all) is limited - even more so in the context of health cards and health search. No previous work has directly investigated the retrieval and ranking of health cards. While related to the general problem of retrieving entity cards, health cards and health search present its own challenges. Effective search is hindered by vocabulary mismatch and lack of domain expertise by users. These issues affect both health search in terms of query formulation and result interpretation and health card retrieval and ranking in terms of matching and deciding the utility of the health card (and thus whether to display the card) $[5,6,18]$. This may explain why, for example, commercial web search engines currently limit the display of health cards to queries that explicitly contain the card's title (i.e., the entity name) [2], although health cards have been shown to be valuable also in the presence of less "navigational" and more explorative health queries [10]. For example, health cards are commonly triggered when the query is a condition such as "meningitis"; while they are not displayed when the user queries using observed symptoms (e.g., "headache fever neck stiff light sensitivity"). This is despite the search results do suggest a relationship between the queries with the observed symptoms and the health condition, e.g., $70 \%$ of the search results in the first Google SERP for the symptoms query above relate to "meningitis".

In this paper we study the problem of ranking health cards in answer to explorative, self-diagnostic consumer health search queries. These are difficult queries for search engines to answer, as they are often underspecified and ambiguous [6]. They are also difficult for users to formulate and appraise results for. Thus, for these queries, the display of relevant high quality health cards could most benefit users' health information acquisition and decision [10]. 
Table 1: Example health scenarios and correct diagnoses. Topic 5, Diagnosis: Deep vein thrombosis Scenario (Topic): Your 65-year-old aunt has had leg pain and swelling over the last 5 days. She has had a high blood pressure, mild congestive heart failure, and recent hospitalization for pneumonia. She had been recovering from the pneumonia at home but when beginning to move around and walk, her right leg became painful, tender, and swollen. Her veins in the right leg are enlarged and her right leg is slightly redder than her left. The back of her knee also fells tender.

Topic 10, Diagnosis: Meningitis

Scenario (Topic): Your 18-year-old nephew is experiencing a very bad headache and fever over the last 3 days. He also complaints of light sensitivity and neck stiffness.

On the other hand, these are queries for which identifying the correct health card(s) to display is particularly challenging. Showing multiple cards may appear to be relevant to the user's ambiguous query as multiple possible diagnoses may be relevant to a medical case, until further analysis of evidence does not lead to the iterative exclusion of the least likely, i.e., the process of differential diagnosis. In this context, we make the following contributions:

(1) We assemble and release the first collection of health cards for research purposes. The considered health cards are related to a large set of queries for medical cases used for evaluation, associated ground truth diagnoses, and other possible diagnoses a user may hypothesise on the basis of (real) search results for the queries.

(2) We empirically evaluate four general entity retrieval methods adapted to the problem of ranking health cards, providing the first quantitative evaluation of entity retrieval techniques in this context. In doing so, we consider specialisations of such techniques to the specific settings of health card retrieval.

\section{METHOD}

\subsection{Creation of Topics and Query Variations}

We used 45 standardised patient vignettes from a survey of symptoms checkers [8] as basis of our health search topics. These vignettes were compiled from various medical sources such as education material for health professionals and a medical resource website. Each vignette contained age, gender, symptoms, correct diagnosis and correct category of triage urgency for a given condition. Of the 45 , we discarded 4 vignettes since there were no health cards in our collection that match their correct diagnosis.

For each vignette, we created a health search task scenario by removing clinical observations which would be not possible for a user to know (e.g., imaging findings, chest auscultations, etc.). We also replaced medical terms with their appropriate layman terms (e.g., "rhinorrhea" was replaced with "runny nose"), to make the scenarios more realistic. Table 1 shows 2 of the 41 scenarios used in this study. Each scenario constituted a topic in our experiment. Each topic has only one relevant health card based on the correct diagnosis for the topic's scenario.

Amazon Mechanical Turk (AMT) workers were recruited to complete HITs based on the 41 topics. In each HIT, we asked workers to use a custom web search engine interface we developed, which mimicked a typical web search engine and allowed users to enter queries and retrieve web pages. Search engine results and snippets were acquired using the Bing Web Search API; only organic results were shown (no advertisement and entity cards). AMT workers were asked to identify the most likely health condition for the given topic and indicate what should the person in the scenario do next (e.g., self-treat, seek attention from a medical professional, etc.).
Table 2: Query variations for topic id $\mathbf{1 0 .}$

\begin{tabular}{ll}
\hline migraine & headache fever light sensitive stiff neck \\
migraines & light sensitivity neck stiffness headache \\
migraine symptoms fever & light sensitivity neck stiffness is it the flu \\
bad headache with fever cause & light sensitivity, headache, stiff neck, fever \\
headache. fever, neck stiffness & $\begin{array}{l}\text { headache fever light sensitivity neck stiffness } \\
\text { headache and fever self treatment } \\
\text { how often do migraines cause fevers }\end{array}$ \\
headache and fever, light sensitivity & $\begin{array}{l}\text { headache fever light sensitivity neck stiffness feber } \\
\text { bad headache and fever light sensitivity and neck } \\
\text { stiffness }\end{array}$ \\
\hline
\end{tabular}

At minimum, workers were asked to submit a query before they could submit their answers. We enforced this by asking workers to mark a search result that they considered as most useful when completing a task. We allowed workers to submit as many queries as required to complete the task.

For each topic, we recruited U.S. based AMT workers; they were paid $\$ 0.2$ for completing a HIT ( 1 HIT $=1$ topic), with a bonus of $\$ 0.5$ for submitting a correct diagnosis ${ }^{1}$. For quality control, initially, we offered 12 HITs for each of the first 10 topics. Then, we evaluated the worker submissions. Workers who submitted poor results were blocked and their submissions discarded. Finally, we sent invitations to the remaining workers to complete the 31 topics left (10 HITs per topic). In total, we accepted 372 submissions for 41 topics (average of 9.1 submissions per topic). Some topics have less than 10 submissions since poor results were discarded and some HITs were left unfinished. $45.58 \%$ of the accepted submissions have the correct diagnosis.

From the 372 submissions, we collected 626 query variations of which 586 were unique, but we removed two queries which failed to retrieve any health cards ("rantidine" and "excema"), thus in total we considered 584 query variations. The average length for unique query variations is $6.78 \pm 5.31$ words. Table 2 shows query variations for topic 10 (see Table 1).

41 out of 584 query variations had "navigational" intent targeting a specific health condition that is not the correct condition for the topic. While these queries are part of the user attempts to identify the correct diagnosis (e.g., "meningitis"), the correct health cards for these queries (query-based relevance), e.g., "migraine", may not be the one for the correct diagnosis (topic-based relevance), e.g., "meningitis". Thus, we considered two types of relevance judgements: topic-based and query-based. For topic-based, the relevant health card is the one that matches the correct diagnosis for the topic. For query-based, the relevant health card is the one that matches the health concept for the query - query-based relevance judgements were considered only for the 41 navigational queries. The query-based judgement results are available online ${ }^{2}$.

\subsection{Creation of the Health Cards Collection}

To create the health cards collection, we crawled pages within the "Diseases and Conditions" sections from Mayo Clinic ${ }^{3}$, as of April 16, 2019. In total, we harvested information for 1,142 health conditions, resulting in as many health cards. Our collection is comparable in size to that of web search engines like Bing ${ }^{4}$. For each condition, we extracted its name (title), aliases, overview, symptoms, and

\footnotetext{
${ }^{1}$ Paid after the diagnosis was verified against the ground truth.

${ }^{2}$ http://ielab.io/publications/jimmy-2019-healthcardretrieval

${ }^{3} \mathrm{https}: / /$ www.mayoclinic.org/diseases-conditions

${ }^{4}$ To verify this, 377.942 health condition phrases extracted from the UMLS were submitted to Bing: 38.958 phrases retrieved a total of 1.330 health cards.
} 
treatments. This ensured that each card contained fielded information similar to those health cards that are commonly shown by commercial search engines, e.g., Google health cards.

\subsection{Considered Ranking Methods}

The health cards catalogue was indexed with respect to the fields title, aliases, overview, symptoms, and treatments. Stop words were removed and Porter stemmer applied.

Then, we considered three common entity ranking models $[9,12$, 16]: BM25F, LM with Dirichlet smoothing and Fielded Sequential Dependence Model (FSDM). In addition, we investigated the effectiveness of Learning to rank (LTR). For this, we used LambdaMart, a listwise ranking algorithm as implemented by QuickRank [4]. As features for LTR, we adopted all term-based features for entity ranking listed by Balog [12]. Then, we added health-entity-based features which are akin to the term-based features. However, instead of terms, we used health concepts to represent queries and health cards. For example, for query length for the health entity feature, instead of counting the number of words in a query, we counted the number of health concepts. To obtain health entities, we used QuickUMLS [14] to map terms in queries and health cards to UMLS concept identifiers. Because of the primarily self-diagnosis intent of the considered topics and search queries, we retained only health entities for which the concept identifiers belonged to the following semantic groups: disorders, chemicals \& drugs, devices, procedures, and anatomy. Finally, we considered features that specifically exploit the characteristics of health cards:

- Sum of the similarity between health entities in query \& field $f$ - Sum of the similarity between health entities in query \& card

These features attempt to measures the similarity between health entities in queries and in cards. We used word2vec clinical concept embeddings [1] to measure the similarity between health entities.

For all methods, we performed parameter tuning using the same 5 -fold cross validation split at topic level. For LTR, for each fold we used $80 \%$ of the training data for learning and $20 \%$ for validation and tuning, with the held out fold used for testing. For BM25F, LM and FSDM, we tuned the field weights between 0 (ignored), 1 , and 2 (twice more important). For BM25F, we tuned the field length normalisation $b$ for long fields (overview, symptoms, and treatments), considering the values $0.25,0.5$, and 0.75 . We did not tune for title and aliases fields as $b$ has minimum impact on short fields. We did not tune $k$ as the term frequency statistic across health cards are unlikely to significantly vary. For LM and FSDM, we tuned $\mu$ for long fields with values 500, 1000, and 1500 .

\subsection{Measures and Statistical Analysis}

We used success at rank 1 (S@1), i.e., how many times the correct health card was retrieved at rank 1 , as primary evaluation metric because it is common practice for current commercial search engines to display only one health card for a query. We also considered S@4 to investigate effectiveness when multiple health cards (4 cards) were shown: this was because of recent work showing the display of multiple cards better supported health search users in exploratory tasks like those considered here [11]. In addition, we considered reciprocal rank (RR) to track at which rank position the correct health card was retrieved: this provides a measure of the gap between the current and the expected performance (i.e., $\mathrm{RR}=1$
Table 3: Experiment results for user queries and scenarios. Win, Tie and Loss show the number of queries that performed better, equal, or worse than BM25F.

\begin{tabular}{|c|c|c|c|c|c|c|}
\hline & \multicolumn{3}{|c|}{ User Queries $(n=584)$} & \multicolumn{3}{|c|}{ Win/Tie/Loss } \\
\hline & S@1 & S@4 & $\mathrm{RR}$ & S@1 & S@4 & $\mathrm{RR}$ \\
\hline$B M 25 F^{a}$ & $.2252^{b C d}$ & $.4426^{B C}$ & $.3411^{B C}$ & - & - & - \\
\hline$L M^{b}$ & $.1798^{a C D}$ & $.3493^{A C D}$ & $.2774^{A C D}$ & $22 / 512 / 50$ & $36 / 457 / 91$ & $164 / 132 / 288$ \\
\hline$F S D M^{c}$ & $.2928^{A B}$ & $.4991^{A B D}$ & $.4003^{A B d}$ & $67 / 488 / 29$ & $55 / 507 / 22$ & $269 / 159 / 156$ \\
\hline \multirow[t]{3}{*}{$L T R^{d}$} & $.2748^{a B}$ & $.4332^{B C}$ & $.3610^{B c}$ & $69 / 476 / 39$ & $51 / 475 / 58$ & $189 / 112 / 283$ \\
\hline & \multicolumn{3}{|c|}{ Scenario Query $(n=41)$} & \multicolumn{3}{|c|}{ Win/Tie/Loss } \\
\hline & S@1 & S@4 & $\mathrm{RR}$ & S@1 & S@4 & $\mathrm{RR}$ \\
\hline$B M 25 F^{a}$ & .3415 & .4634 & .4362 & - & - & - \\
\hline$L M^{b}$ & .2927 & .4390 & .3909 & $2 / 35 / 4$ & $1 / 38 / 2$ & $12 / 13 / 16$ \\
\hline$F S D M^{c}$ & .2195 & .5122 & .3796 & $1 / 34 / 6$ & $5 / 33 / 3$ & $13 / 11 / 17$ \\
\hline$L T R^{d}$ & .2195 & .3658 & .3015 & $3 / 30 / 8$ & $2 / 33 / 6$ & $6 / 6 / 29$ \\
\hline
\end{tabular}

means the correct card was successfully retrieved at rank 1). Finally, we also computed session-based success measures; i.e., whether the correct health card for the search task was displayed at the targeted ranks at any point throughout a user's search session.

Statistical significant differences were measured using pairwise t-test with Bonferroni correction. These are reported in results tables using lower case superscripts for $\alpha<0.05$ and upper case for $\alpha<0.01$.

\section{EXPERIMENT RESULTS}

\subsection{Retrieval performance}

Table 3 reports the retrieval results obtained by the different methods. Along with the results for user queries, we also report (for completeness) the results obtained when the verbose (layman) scenario was used as the query. While it is not expected users would query with the complete scenario in real situations, we observe that the best methods on the scenarios obtain higher effectiveness than the best methods on the user queries. This is understandable as the user queries generally contain less details than the scenarios, highlighting both the difficulties by users in formulating effective queries and by systems in retrieving the correct cards.

In terms of overall retrieval effectiveness on realistic user queries, methods based on FSDM and LTR are comparable (with FSDM being generally better than LTR); both significantly better than LM, while only FSDM exhibits statistical significant differences with BM25F. This may be because FSDM does explicitly address expressions with sequential words that are commonly seen in the user queries (e.g., "neck stiffness" in Table 2), while BM25F does not.

Table 3 also reports a summary of query-by-query comparison between each method and BM25F (e.g., a win: better than BM25F on one query). This shows that while FSDM and LTR achieve a similar number of wins over BM25F for S@1 and S@4 (for user queries), LTR does have more losses.

\subsection{Search session based evaluation}

Within the search sessions obtained via AMT, users submitted multiple queries when completing a task (Section 2.1). Different search styles were displayed. For example, Table 5 reports a querying session of a user, displaying progressive reformulation of queries as the session goes on. In this example, the user concluded the search task with a clear query and obtained the information which lead them making the correct diagnosis. 
Table 4: Session-based results: success is measured over the whole search session for a single user, rather than for each query separately.

\begin{tabular}{lllll}
\hline \multicolumn{2}{c}{ User Sessions (n=372) } & \multicolumn{2}{c}{ Win/Tie/Loss } \\
S@1 & S@4 & S@1 & S@4 \\
\hline$B M 25 F^{a}$ & $.2997^{b d}$ & $.5269^{B C}$ & - & - \\
\hline$L M^{b}$ & $.2406^{a C D}$ & $.4462^{A C d}$ & $18 / 313 / 41$ & $22 / 297 / 53$ \\
\hline$F S D M^{c}$ & $.3548^{B}$ & $.5860^{A B D}$ & $49 / 294 / 29$ & $34 / 326 / 12$ \\
\hline$L T R^{d}$ & $.3683^{a B}$ & $.5134^{b C}$ & $57 / 286 / 29$ & $34 / 298 / 40$ \\
\hline
\end{tabular}

Table 5: A query session and simulated card retrieval effectiveness for Scenario 38 (correct diagnosis: "canker sore").

\begin{tabular}{|c|c|c|c|c|}
\hline & $\begin{array}{l}q_{1} \\
\text { mouth ulceration }\end{array}$ & $\begin{array}{l}\mathrm{q} 2 \\
\text { mouth herpes }\end{array}$ & $\begin{array}{l}\text { q3 } \\
\text { mouth herpes ulcer }\end{array}$ & $\begin{array}{l}\mathrm{q} 4 \\
\text { canker sore }\end{array}$ \\
\hline S@1 & 0.0000 & 0.0000 & 0.0000 & 1.0000 \\
\hline$\overline{S @ 4}$ & 0.0000 & 0.0000 & 1.000 & 1.0000 \\
\hline $\mathrm{RR}$ & 0.1667 & 0.1429 & 0.5000 & 1.0000 \\
\hline
\end{tabular}

Table 6: Experiment results for user queries using LTR with only basic features (42 Features) vs. LTR with also health specific features (90 features). * and ${ }^{* *}$ indicates statistical significance difference with regard to LTR-basic at $\alpha<0.05$ and $\alpha<0.01$ levels.

\begin{tabular}{lllllll}
\hline & \multicolumn{3}{c}{ User Queries } & $(\mathbf{n}=\mathbf{5 8 4})$ & \multicolumn{3}{c}{ Win/Tie/Loss } \\
& $\mathrm{S} @ 1$ & $\mathrm{~S} @ 4$ & $\mathrm{RR}$ & $\mathrm{S} @ 1$ & $\mathrm{~S} @ 4$ & $\mathrm{RR}$ \\
LTR-basic & .2166 & .3947 & .3187 & - & - & - \\
LTR & $.2748^{* *}$ &. $\mathbf{4 3 3 2}$ &. $\mathbf{3 6 1 0}$ & $66 / 488 / 32$ & $65 / 478 / 43$ & $174 / 116 / 296$ \\
\hline
\end{tabular}

This motivated us to investigate session-based evaluation - i.e., consider the effectiveness of methods over a whole session for each single users. We report session-based evaluation results in Table 4 for S@1,4. These results show that over a session, LTR is better than other methods at retrieving the correct card at rank 1 (although significant differences are found only with respect to LM); if multiple (4) cards were displayed, then FSDM is best.

\subsection{Features importance for Learning to Rank}

RankEval [13] was used to study the effect different features had on LTR performance; the tool ranks features based on their importance and usage statistics. Figure 1 reports the top ten most important features for each query set based on importance gain. This analysis suggests that for LTR, the features we introduced based on specific health cards characteristics (entity based representation and entity similarities) are amongst the features that contribute most to the LTR effectiveness.

We further investigate the gains obtained in LTR from including health cards specific features as opposed to considering only the generic features for entity retrieval [12](LTR-basic). Table 6 shows that the health card features we introduced in this work provide significant gains in performance, with the LTR using basic features even performing worse than BM25F.

\section{CONCLUSION}

In this paper, we empirically investigated methods to retrieve health cards in the context of consumer health search with self-diagnosis intents. Queries were often ambiguous and generally difficult, as they often did not contain an explicit mention of the target health card title. As part of this investigation, we assembled the first test

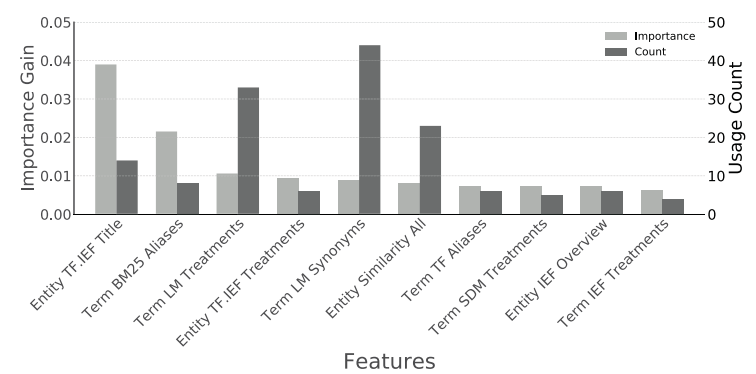

Figure 1: Feature importance for LTR on user queries.

collection of health cards containing information for 1,142 health conditions. Furthermore, we collected 373 search session for a total of 626 queries (586 unique) for 41 self-diagnosis search tasks. This data is released to the research community ${ }^{5}$.

The retrieval methods considered were BM25F, LM, FSDM and LTR. Our results suggest that FSDM and LTR are comparable and performed best across a large set of realistic user queries for the task at hand. For LTR, we introduced features specific to health cards (health entities and health entities similarities), which were found to have a statistically strong impact on the effectiveness of this method.

Acknowledgments: Jimmy is sponsored by the Indonesia Endowment Fund for Education (Lembaga Pengelola Dana Pendidikan / LPDP) (20151022014644). Guido Zuccon is the recipient of an Australian Research Council DECRA Research Fellowship (DE180101579) and a Google Faculty Award; both these grants funded this study.

\section{REFERENCES}

[1] Beam AL, Kompa B, Fried I, Palmer NP, Shi X, Cai T, and Kohane IS. 2018. Clinical concept embeddings learned from massive sources of medical data. arXiv preprint arXiv:1804.01486 (2018).

[2] Gabrilovich E. 2016. Cura Te Ipsum: answering symptom queries with question intent. In Second WebQA workshop, SIGIR 2016 (invited talk).

[3] Radlinski F, Craswell N, Billerbeck B, Shokouhi M, Ahari S, Agrawal N, Hoad T, Zhou S, and Awan MA. 2015. Entity detection and extraction for entity cards. US Patent 9,158,846.

[4] Capannini G, Lucchese C, Nardini FM, Orlando S, Perego R, and Tonellotto N. 2016. Quality versus efficiency in document scoring with learning-to-rank models. IP\&M 52, 6 (2016), 1161-1177.

[5] Zuccon G and Koopman B. 2018. SIGIR 2018 Tutorial on Health Search (HS2018): A Full-day from Consumers to Clinicians. In SIGIR'18. ACM, 1391-1394.

[6] Zuccon G, Koopman B, and Palotti J. 2015. Diagnose this if you can. In ECIR'15.

[7] Bota H, Zhou K, and Jose JM. 2016. Playing your cards right: The effect of entity cards on search behaviour and workload. In CHIIR'16.

[8] Semigran HL, Linder JA, Gidengil C, and Mehrotra A. 2015. Evaluation of symptom checkers for self diagnosis and triage: audit study. BM7 351 (2015).

[9] Chen J, Xiong C, and Callan J. 2016. An empirical study of learning to rank for entity search. In SIGIR'16. ACM, 737-740.

[10] Jimmy, Zuccon G, Koopman B, and Demartini G. 2019. Health Cards for Consumer Health Search. In SIGIR'19.

[11] Jimmy, Zuccon G, Demartini G, and Koopman B. 2019. Health Cards to Assist Decision Making in Consumer Health Search. In AMIA'19.

[12] Balog K. 2018. Entity-oriented search. Springer.

[13] Claudio L, Cristina IM, Franco MN, Raffaele P, and Salvatore T. 2017. RankEval: An Evaluation and Analysis Framework for Learning-to-Rank Solutions. In SIGIR'2017.

[14] Soldaini L and Goharian N. 2016. QuickUMLS: a fast, unsupervised approach for medical concept extraction. In SIGIR MedIR Workshop.

[15] Shokouhi M and Guo Q. 2015. From Queries to Cards: Re-ranking Proactive Card Recommendations Based on Reactive Search History. In SIGIR'15.

[16] Zhiltsov N, Kotov A, and Nikolaev F. 2015. Fielded sequential dependence model for ad-hoc entity retrieval in the web of data. In SIGIR'15.

[17] Thomas P, Moffat A, Bailey P, Scholer F, and Craswell N. 2018. Better Effectiveness Metrics for SERPs, Cards, and Rankings. In ADCS '18.

[18] Zeng Q, Kogan S, Ash N, Greenes RA, and Boxwala AA. 2002. Characteristics of consumer terminology for health information retrieval. Methods of Information in Medicine 41, 4 (2002), 289-298.

$\overline{{ }^{5} \text { http://ielab.io/publications/jimmy-2019-healthcardretrieval }}$ 
Association for

Computing Machinery

November 3-7, 2019

Beijing, China

Advancing Computing as a Science \& Profession

\section{CIKM '19}

Proceedings of the 28th ACM International Conference on Information \& Knowledge Management

Sponsored by:

ACM SIGIR \& ACM SIGWEB

General Chairs:

Wenwu Zhu, Dacheng Tao, \& Xueqi Cheng

Program Chairs:

Peng Cui, Elke Rundensteiner, David Carmel, Qi He, \& Jeffrey Xu Yu

Publication Chairs:

Meng Jiang \& Mu Qiao 
Copyright $\odot 2019$ by the Association for Computing Machinery, Inc. (ACM). Permission to make digital or hard copies of portions of this work for personal or classroom use is granted without fee provided that copies are not made or distributed for profit or commercial advantage and that copies bear this notice and the full citation on the first page. Copyright for components of this work owned by others than ACM must be honored. Abstracting with credit is permitted. To copy otherwise, to republish, to post on servers or to redistribute to lists, requires prior specific permission and/or a fee. Request permission to republish from: permissions@acm.org or Fax +1 (212) 869-0481.

For other copying of articles that carry a code at the bottom of the first or last page, copying is permitted provided that the per-copy fee indicated in the code is paid through www.copyright.com.

ISBN: 978-1-4503-6976-3

Additional copies may be ordered prepaid from:

ACM Order Department

PO Box 30777

New York, NY 10087-0777, USA
Phone: 1-800-342-6626 (USA and Canada)

$+1-212-626-0500$ (Global)

Fax: +1-212-944-1318

E-mail: acmhelp@acm.org

Hours of Operation: 8:30 am - 4:30 pm ET

Printed in the USA 


\section{Welcome from General Chairs}

It is my great honour to welcome you to CIKM 2019, the 28th ACM International Conference on Information and Knowledge Management, will be held in Beijing, China from the 3rd to the 7th of November 2019. Beijing, the capital city of China, is a major historical and cultural centre, as well as China's largest science and technology hub.

CIKM is an annual conference in the computer science research field. Since it was first held in the United States in 1992, 27 conferences have been held in 8 countries around the world. The research areas it primarily focuses on include database management, information retrieval, and knowledge management. It has been evolving into a major conference in these research areas. Our theme this year is "AI for future life". Since the term "artificial intelligence" was first proposed by John McCarthy in 1955, artificial intelligence has been evolving for more than half a century. It is gaining greater significance in our daily lives, and the importance of scientific research in the area is becoming increasingly prominent.

We are proud to present four distinguished keynote speakers to CIKM 2019 attendees: Steve Maybank, Jiawei Han, Jian Pei and Jianping Shi. We are also honored to have about ten spectacular industry talks in this year's conference. To allow the participants to better understand the most cutting-edge work in today's research field, we will arrange 9 tutorials, 9 workshops, and 26 demos. World renowned experts from academia and industry have been invited to give keynote speeches to showcase and disseminate the latest research outcome in related research fields.

We are honored to announce that this conference has received 1676 submissions. All these submissions were assigned to qualified reviewers and area chairs for review and acceptance. Reviewers and area chairs were selected by Program Committee Chairs through checking their research fields and publications in recent years to avoid conflict with their work throughout the review process. Finally, 202 long papers, 107 short papers and 37 applied research papers were accepted. The number of long, short and applied research papers account for $21 \%$ of the total number of accepted papers. The proceedings of CIKM 2019 will be published by ACM.

We wish to thank the organizers and reviewers who provided their time and effort to facilitate this event, every enthusiastic speaker, every participant come from all over the world to attend the event. We also want to express thankfulness for our awesome organizing committee for their great efforts in supporting the conference. Last but not least, we would also like to thank all the sponsors for their warm support, your generous donation will be apricated. Finally, we wish you all have fruitful connections with the community, we wish CIKM2019 all success.

We look forward to seeing you in Beijing. We wish CIKM2019 will bring memorable experiences to our lives.

Wenwu Zhu

Tsinghu University, China
Dacheng Tao

The University of Sydney, Australia 


\title{
Welcome from Program Chairs
}

It is our great pleasure to welcome you to the 28th ACM International C1onference on Information and Knowledge Management (CIKM 2019), held in Beijing, China, from November 3-7, 2019. CIKM is a top-tier ACM conference in the areas of information retrieval, knowledge management and databases. Since 1992, it has successfully brought together leading researchers and developers from the three communities, with the purpose of identifying challenging problems facing the development of advanced knowledge and information systems, and shaping future research directions through the publication of high quality, applied and theoretical research findings.

CIKM 2019 continues to create an outstanding technical program including presentations and posters for long and short research papers, applied research papers and demo papers. In total, we have received 1,031 full paper (10 pages) submissions and 471 short paper (4 pages) submissions reviewed by a group of 90 senior program committee members and 588 program committee members. The research track accepted 202 full papers with acceptance rate as $19.6 \%$. 107 short submissions were accepted as short papers (4 pages) with acceptance rate as $22.7 \%$. In order to promote the application and impact of frontier AI and big data technologies in real industrial scenarios, CIKM 2019 features a new track, named Applied Research Track, to attract submissions from both industry and academia that either solve or advance the understanding of issues related to deploying AI and big data technologies in the real world. We have received 174 submissions this year, and 38 submissions were accepted with acceptance rate as $21.8 \%$. In total, we have received 1,676 paper submissions in research track, which is a record-breaking number.

Besides, CIKM2019 features a broad spectrum of research topics in workshops, tutorials, and demos. We provide 9 co-located workshops, ranging from transportation to bioinformatics, from recommender system to human quality of life. We also provide 6 conventional tutorials covering knowledge graph, social network, database systems etc., and 3 hands-on tutorials in deep learning, recommendation and graph learning. At the same time, there are 26 highly selected demos to demonstrate the power and impact of information technology.

We are grateful to everyone who made this technical program possible. First of all, we would like to thank all the contributing authors who submitted their excellent work. The conference would not exist without your contributions. Second, we would in particular like to recognize the immense efforts of the workshop chairs, tutorial chairs, demo chairs, senior program committee members, program committee members, and external reviewers, who selected the technical program with their great expertise. Finally, we hope to thank all our corporate sponsors for their generous support.

We hope you enjoy the program and the opportunity to interact and collaborate with friends and colleagues from around the world.

\section{Peng Cui}

Tsinghua University, China

Elke Rundensteiner

Worcester Polytechnic Institute, USA

\section{David Carmel}

Amazon Research, Israel

Qi He

LinkedIn, USA

\author{
Jeffrey Xu Yu \\ Chinese University of Hong Kong, China
}




\section{Table of Contents}

CIKM '19 Conference Organization $\mathrm{xxx} 1$

CIKM '19 Senior Program Committee - Long Track $\mathrm{xxx} 2$

CIKM '19 Program Committee Members - Long Track ................................................ xxx3

CIKM '19 Program Committee Members - Short Track ................................................. xxx4

CIKM '19 Program Committee Members - Applied Track ……………………........... xxx5

CIKM '19 Program Committee Members - Demo Track ............................................... xxx6

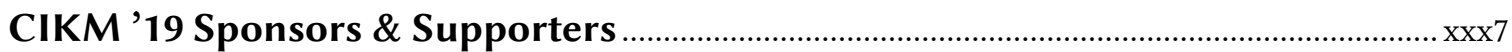

\section{Keynote Addressess}

- Autonomous Driving Towards Mass Production.

Jianping Shi (SenseTime Group Ltd)

- The Fisher-Rao Metric in Computer Vision

Steve Maybank (Birkbeck College \& University of London)

- From Unstructured Text to TextCube: Automated Construction and Multidimensional Exploration

Jiawei Han (University of Illinois at Urbana-Champaign)

- Practicing the Art of Data Science 7 Jian Pei (Simon Fraser University)

\section{Session: Long - Algorithmic Techniques}

- On VR Spatial Query for Dual Entangled Worlds .

Shao-Heng Ko (Academia Sinica), Ying-Chun Lin (Purdue University),

Hsu-Chao Lai (National Chiao Tung University \& Academia Sinica),

Wang-Chien Lee (The Pennsylvania State University), De-Nian Yang (Academia Sinica)

- Sketching Streaming Histogram Elements using Multiple Weighted Factors . 19 Quang-Huy Duong, Heri Ramampiaro, Kjetil Nørvåg (Norwegian University of Science and Technology)

- Improved Compressed String Dictionaries

Nieves R. Brisaboa, Ana Cerdeira-Pena, Guillermo de Bernardo

(Universidade da Coruña - Centro de Investigación CITIC),

Gonzalo Navarro (University of Chile)

- On Transforming Relevance Scales.

Lei Han (University of Queensland), Kevin Roitero (University of Udine),

Eddy Maddalena (University of Southampton), Stefano Mizzaro (University of Udine),

Gianluca Demartini (University of Queensland)

- Streamline Density Peak Clustering for Practical Adoptions Shuai Yang, Xipeng Shen, Min Chi (North Carolina State University)

\section{Session: Long - Analyzing Spatio-Temporal Data}

- Recommendation-based Team Formation for On-demand Taxi-calling Platforms. 59 Lingyu Zhang (Didi Chuxing), Tianshu Song, Yongxin Tong (Beihang University), Zimu Zhou (ETH Zurich), Dan Li (Didi Chuxing), Wei Ai (University of Michigan), Lulu Zhang, Guobin Wu (Didi Chuxing), Yan Liu (University of Southern California), Jieping Ye (Didi Chuxing)

- DeepIST: Deep Image-based Spatio-Temporal Network for Travel Time Estimation 69 Tao-yang Fu, Wang-chien Lee (The Pennsylvania State University) 
- Personalized Route Description Based On Historical Trajectories.

Han Su, GuangLin Cong, Wei Chen (University of Electronic Science and Technology of China Chengdu, China),

Bolong Zheng (Huazhong University of Science and Technology),

Kai Zheng (University of Electronic Science and Technology of China Chengdu, China)

- Geolocating Tweets in any Language at any Location

Mike Izbicki (Claremont McKenna College),

Vagelis Papalexakis, Vassilis Tsotras (University of California, Riverside)

- SeiSMo: Semi-supervised Time Series Motif Discovery for Seismic Signal Detection

M Ashraf Siddiquee, Zeinab Akhavan, Abdullah Mueen (University of New Mexico)

\section{Session: Long - Biomedical Informatics}

- UA-CRNN: Uncertainty-Aware Convolutional Recurrent Neural Network for Mortality Risk Prediction

Qingxiong Tan (Hong Kong Baptist University), Andy Jinhua Ma (Sun Yat-Sen University),

Mang Ye, Baoyao Yang (Hong Kong Baptist University),

Huiqi Deng (Hong Kong Baptist University \& Sun Yat-Sen University),

Vincent Wai-Sun Wong, Yee-Kit Tse, Terry Cheuk-Fung Yip, Grace Lai-Hung Wong,

Jessica Yuet-Ling Ching, Francis Ka-Leung Chan (The Chinese University of Hong Kong),

Pong C. Yuen (Hong Kong Baptist University)

- Learning More with Less: Conditional PGGAN-based Data Augmentation for Brain Metastases Detection Using Highly-Rough Annotation on MR Images

Changhee Han (National Institute of Informatics, National Center for Global Health and Medicine, \& University

of Cambridge), Kohei Murao (National Institute of Informatics \& National Center for Global Health and Medicine),

Tomoyuki Noguchi, Yusuke Kawata, Fumiya Uchiyama (National Center for Global Health and Medicine),

Leonardo Rundo (University of Cambridge), Hideki Nakayama (The University of Tokyo),

Shin'ichi Satoh (National Institute of Informatics \& The University of Tokyo)

- Domain Knowledge Guided Deep Atrial Fibrillation Classification and Its Visual Interpretation.

Xiaoyu Li, Buyue Qian (Xi'an fiaotong University), Jishang Wei (HP Lab),

Xianli Zhang, Sirui Chen, Qinghua Zheng (Xi'an fiaotong University)

- Question Difficulty Prediction for Multiple Choice Problems in Medical Exams.

Zhaopeng Qiu, Xian Wu, Wei Fan (Tencent Medical AI Lab)

- GRAPHENE: A Precise Biomedical Literature Retrieval Engine with Graph Augmented Deep Learning and External Knowledge Empowerment.

Sendong Zhao, Chang Su, Andrea Sboner, Fei Wang (Cornell University)

\section{Session: Long - Computer Vision}

- Video-level Multi-model Fusion for Action Recognition

Xiaomin Wang, Junsan Zhang, Leiquan Wang (China University of Petroleum (EastChina)),

Philip S. Yu (University of Illinois at Chicago), Jie Zhu (The National Police University for Criminal fustice),

Haisheng Li (Beijing Technology and Business University)

- Large Scale Landmark Recognition via Deep Metric Learning

Andrei Boiarov, Eduard Tyantov (Mail.ru Group)

- Multi-stage Deep Classifier Cascades for Open World Recognition

Xiaojie Guo, Amir Alipour-Fanid (George Mason University), Lingfei Wu (IBM Research AI),

Hemant Purohit, Xiang Chen, Kai Zeng, Liang Zhao (George Mason University)

- Inferring Context from Pixels for Multimodal Image Classification

Manan Shah (Stanford University \& Google Research),

Krishnamurthy Viswanathan, Chun-Ta Lu, Ariel Fuxman, Zhen Li (Google Research),

Aleksei Timofeev (Waymo), Chao Jia, Chen Sun (Google Research)

- Multi-Target Multi-Camera Tracking with Human Body Part Semantic Features 199

Mingkun Wang (National University of Defense Technology),

Dianxi Shi, Naiyang Guan, Wei Yi (National Innovation Institute of Defense Technology

\& Tianjin Artificial Intelligence Innovation Center), Tao Zhang (University of Illinois at Chicago),

Zunlin Fan (National Innovation Institute of Defense Technology \& Tianjin Artificial Intelligence Innovation Center), 


\section{Session: Long - Database and System}

- Efficient Join Processing Over Incomplete Data Streams

Weilong Ren, Xiang Lian (Kent State University),

Kambiz Ghazinour (State University of New York \& Kent State University)

- Inclusion Dependency Discovery: An Experimental Evaluation of Thirteen Algorithms

Falco Dürsch, Axel Stebner, Fabian Windheuser, Maxi Fischer, Tim Friedrich,

Nils Strelow, Tobias Bleifuß, Hazar Harmouch, Lan Jiang, Thorsten Papenbrock,

Felix Naumann (Hasso Plattner Institute)

- Constructing a Comprehensive Events Database from the Web.

Qifan Wang, Bhargav Kanagal, Vijay Garg, D. Sivakumar (Google Research)

- Deploying Hash Tables on Die-Stacked High Bandwidth Memory

Xuntao Cheng (Alibaba Group), Bingsheng He (National University of Singapore),

Eric Lo (The Chinese University of Hong Kong),

Wei Wang, Shengliang Lu, Xinyu Chen (National University of Singapore)

\section{Session: Long - Domain Adaptation and Transfer Learning}

- Partially Shared Adversarial Learning For Semi-supervised Multi-platform User Identity Linkage

Chaozhuo Li (Beihang University), Senzhang Wang (Nanjing University of Aeronautics and Astronautics),

Hao Wang (Southwest fiaotong University), Yanbo Liang (Facebook),

Philip S. Yu (University of Illinois at Chicago), Zhoujun Li (Beihang University),

Wei Wang (Ping An Bank Co., Ltd.)

- Adversarial Domain Adaptation with Semantic Consistency for Cross-Domain

Image Classification

Manliang Cao, Xiangdong Zhou, Yiming Xu, Yue Pang, Bo Yao (Fudan University)

- ATL: Autonomous Knowledge Transfer from Many Streaming Processes

Mahardhika Pratama, Marcus de Carvalho, Renchunzi Xie (Nanyang Technological University),

Edwin Lughofer (Fohannes Kepler University), Jie Lu (University of Technology Sydney)

- Knowledge Transfer based on Multiple Manifolds Assumption

Pengfei Wei (National University of Singapore), Yiping Ke (Nanyang Technological University)

- Cross-domain Aspect Category Transfer and Detection via Traceable Heterogeneous Graph Representation Learning .

Zhuoren Jiang (Sun Yat-sen University), Jian Wang, Lujun Zhao, Changlong Sun (Alibaba Group),

Yao Lu (Sun Yat-sen University), Xiaozhong Liu (Indiana University Bloomington)

\section{Session: Long - E-Commerce and Advertising I}

- A Deep Neural Framework for Sales Forecasting in E-Commerce

Yan Qi, Chenliang Li (Wuhan University), Han Deng, Min Cai, Yunwei Qi, Yuming Deng (Alibaba Group)

- An Active and Deep Semantic Matching Framework for Query Rewrite in E-Commercial Search Engine .....

Yatao Yang (Sun Yat-sen University), Jun Tan, Hongbo Deng (Alibaba Group),

Zibin Zheng, Yutong Lu (Sun Yat-sen University), Xiangke Liao (National University of Defense Technology)

- AIBox: CTR Prediction Model Training on a Single Node

Weijie Zhao, Jingyuan Zhang (Baidu Research USA), Deping Xie, Yulei Qian, Ronglai Jia (Baidu Inc.),

Ping Li (Baidu Research USA)

- Improving Ad Click Prediction by Considering Non-displayed Events

Bowen Yuan, Jui-Yang Hsia (National Taiwan University), Meng-Yuan Yang (ETH Zurich),

Hong Zhu, Chih-Yao Chang, Zhenhua Dong (Huawei Noah's ark lab), Chih-Jen Lin (National Taiwan University)

- Approximation Algorithms for Coordinating Ad Campaigns on Social Networks 


\section{Session: Long - E-Commerce and Advertising II}

- Regularized Adversarial Sampling and Deep Time-aware Attention for Click-Through Rate Prediction

Yikai Wang (Tsinghua University), Liang Zhang (FD.COM),

Quanyu Dai (The Hong Kong Polytechnic University), Fuchun Sun (Tsinghua University),

Bo Zhang, Yang He, Weipeng Yan, Yongjun Bao (FD.COM)

- Conversational Product Search Based on Negative Feedback

Keping Bi, Qingyao Ai (University of Massachusetts, Amherst), Yongfeng Zhang (Rutgers University), W. Bruce Croft (University of Massachusetts, Amherst)

- Learning to Ask: Question-based Sequential Bayesian Product Search

Jie Zou, Evangelos Kanoulas (University of Amsterdam),

- A Zero Attention Model for Personalized Product Search.

Qingyao Ai (University of Utah), Daniel N. Hill, S. V. N. Vishwanathan (Amazon Search),

W. Bruce Croft (University of Massachusetts, Amherst)

- Learning to Generate Personalized Product Descriptions

Guy Elad (Technion), Ido Guy, Slava Novgorodov (eBay Research), Benny Kimelfeld, Kira Radinsky (Technion)

\section{Session: Long - Network Embedding I}

- Fast and Accurate Network Embeddings via Very Sparse Random Projection

Haochen Chen, Syed Fahad Sultan, Yingtao Tian (Stony Brook University),

Muhao Chen (University of California, Los Angeles), Steven Skiena (Stony Brook University)

- Hierarchical Community Structure Preserving Network Embedding:

A Subspace Approach

Qingqing Long, Yiming Wang (Peking University), Lun Du (Microsoft Research \& Peking University),

Guojie Song, Yilun Jin (Peking University), Wei Lin (Alibaba Group),

- Collective Link Prediction Oriented Network Embedding with Hierarchical Graph Attention

Yizhu Jiao, Yun Xiong (Fudan University), Jiawei Zhang (Florida State University),

Yangyong Zhu (Fudan University)

- Discerning Edge Influence for Network Embedding ...

Yaojing Wang, Yuan Yao (Nanjing University),

Hanghang Tong (University of Illinois at Urbana-Champaign \& Arizona State University),

Feng Xu, Jian Lu (Nanjing University)

- Constrained Co-embedding Model for User Profiling in Question

Answering Communities....

Yupeng Luo, Shangsong Liang (Sun Yat-sen University), Zaiqiao Meng (University of Glasgow),

\section{Session: Long - Network Embedding II}

- Hyper-Path-Based Representation Learning for Hyper-Networks

Jie Huang (Sun Yat-sen University), Xin Liu, Yangqiu Song (The Hong Kong University of Science and Technology)

- Multi-Hot Compact Network Embedding

Chaozhuo Li (Beihang University), Lei Zheng (University of Illinois at Chicago),

Senzhang Wang (Nanjing University of Aeronautics and Astronautics), Feiran Huang (finan University),

Philip S. Yu (University of Illinois at Chicago), Zhoujun Li (Beihang University)

- Temporal Network Embedding with Micro- and Macro-dynamics.

Yuanfu Lu, Xiao Wang, Chuan Shi (Beijing University of Posts and Communications),

Philip S. Yu (University of Illinois at Chicago), Yanfang Ye (Case Western Reserve University),

- MrMine: Multi-resolution Multi-network Embedding ....

Boxin Du, Hanghang Tong (University of Illinois at Urbana-Champaign)

- Task-Guided Pair Embedding in Heterogeneous Network

Chanyoung Park (University of Illinois at Urbana-Champaign), Donghyun Kim (Yahoo Research),

Qi Zhu, Jiawei Han (University of Illinois at Urbana-Champaign),

Hwanjo Yu (Pohang University of Science and Technology) 


\section{Session: Long - Graph Nerual Network I}

- Graph Convolutional Networks with Motif-based Attention

John Boaz Lee (Worcester Polytechnic Institute), Ryan A. Rossi (Adobe Research),

Xiangnan Kong (Worcester Polytechnic Institute), Sungchul Kim, Eunyee Koh, Anup Rao (Adobe Research)

- Long-tail Hashtag Recommendation for Micro-videos with Graph

Convolutional Network

Mengmeng Li, Tian Gan, Meng Liu (Shandong University),

Zhiyong Cheng (Qilu University of Technology (Shandong Academy of Sciences)),

Jianhua Yin, Liqiang Nie (Shandong University)

- Hashing Graph Convolution for Node Classification

Wenting Zhao, Zhen Cui, Chunyan Xu, Chengzheng Li, Tong Zhang, Jian Yang (Jiangsu Key Lab of Image

and Video Understanding for Social Security \& Nanjing University of Science and Technology)

- Relation-Aware Graph Convolutional Networks for Agent-Initiated Social E-Commerce Recommendation 529

Fengli Xu (Tsinghua University), Jianxun Lian (Miscrosoft Research Asia),

Zhenyu Han, Yong Li (Tsinghua University), Yujian Xu (Beibei Group), Xing Xie (Microsoft Research Asia)

- Fi-GNN: Modeling Feature Interactions via Graph Neural Networks for CTR Prediction ........ 539 Zekun Li, Zeyu Cui, Shu Wu (Institute of Information Engineering, Chinese Academy of Sciences \& University of Chinese Academy of Sciences),

Xiaoyu Zhang (Institute of Information Engineering, Chinese Academy of Sciences),

Liang Wang (Institute of Information Engineering, Chinese Academy of Sciences \& University

of Chinese Academy of Sciences)

\section{Session: Long - Graph Nerual Network II}

- Key Player Identification in Underground Forums over Attributed Heterogeneous Information Network Embedding Framework.

Yiming Zhang, Yujie Fan, Yanfang Ye (Case Western Reserve University),

Liang Zhao (George Mason University), Chuan Shi (Beijing University of Posts and Telecommunications)

- Learning to Identify High Betweenness Centrality Nodes from Scratch:

A Novel Graph Neural Network Approach

Changjun Fan (National University of Defense Technology \& University of California, Los Angeles),

Li Zeng (National University of Defense Technology), Yuhui Ding (Tsinghua University),

Muhao Chen (University of California, Los Angeles \& University of Pennsylvania),

Yizhou Sun (University of California, Los Angeles), Zhong Liu (National University of Defense Technology)

- Multiple Rumor Source Detection with Graph Convolutional Networks

Ming Dong, Bolong Zheng (Huazhong University of Science and Technology),

Nguyen Quoc Viet Hung (Griffith University),

Han Su (University of Electronic Science and Technology of China),

Guohui Li (Huazhong University of Science and Technology)

- Rethinking the Item Order in Session-based Recommendation with Graph Neural Networks.

Ruihong Qiu (The University of Queensland), Jingjing Li (University of Electronic Science and Technology of China),

Zi Huang, Hongzhi YIn (The University of Queensland)

- Gravity-Inspired Graph Autoencoders for Directed Link Prediction

Guillaume Salha (Deezer Research \& Development \& École Polytechnique), Stratis Limnios (École Polytechnique), Romain Hennequin, Viet-Anh Tran (Deezer Research \& Development), Michalis Vazirgiannis (École Polytechnique)

\section{Session: Long - Heterogeneous Data}

- Discovering Hypernymy in Text-Rich Heterogeneous Information Network

Yu Shi, Jiaming Shen, Yuchen Li, Naijing Zhang, Xinwei He, Zhengzhi Lou,

Qi Zhu (University of Illinois Urbana-Champaign), Matthew Walker (LinkedIn Corporation),

Myunghwan Kim (Mesh Korea), Jiawei Han (University of Illinois Urbana-Champaign) 
- $\alpha$ Cyber: Enhancing Robustness of Android Malware Detection System against Adversarial Attacks on Heterogeneous Graph based Model Shifu Hou, Yujie Fan, Yiming Zhang, Yanfang Ye (Case Western Reserve University), Jingwei Lei, Wenqiang Wan, Jiabin Wang, Qi Xiong, Fudong Shao (Tencent Security Lab)

- BHIN2vec: Balancing the Type of Relation in Heterogeneous Information Network 619 Seonghyeon Lee (Pohang University of Science and Technology), Chanyoung Park (University of Illinois at Urbana-Champaign), Hwanjo Yu (Pohang University of Science and Technology)

- Deep Sequence-to-Sequence Entity Matching for Heterogeneous Entity Resolution 629

Hao Nie (Institute of Software, Chinese Academy of Sciences \& University of Chinese Academy of Sciences), Xianpei Han (Institute of Software, Chinese Academy of Sciences),

Ben He (Institute of Software, Chinese Academy of Sciences \& University of Chinese Academy of Sciences), Le Sun, Bo Chen (Institute of Software, Chinese Academy of Sciences),

Wei Zhang, Suhui Wu, Hao Kong (Alibaba Group)

- HeteSpaceyWalk: A Heterogeneous Spacey Random Walk for Heterogeneous Information Network Embedding

Yu He (Beijing Advanced Innovation Center for Big Data and Brain Computing \& Beihang University), Yangqiu Song (Hong Kong University of Science and Technology),

Jianxin Li, Cheng Ji (Beijing Advanced Innovation Center for Big Data and Brain Computing \& Beihang University), Jian Peng (University of Illinois at Urbana-Champaign),

Hao Peng (Beijing Advanced Innovation Center for Big Data and Brain Computing \& Beihang University)

\section{Session: Long - Knowledge Graph I}

- EHR Coding with Multi-scale Feature Attention and Structured Knowledge Graph Propagation

Xiancheng Xie, Yun Xiong (Fudan University), Philip S. Yu (University of Illinois at Chicago),

Yangyong Zhu (Fudan University)

- A Fine-grained and Noise-aware Method for Neural Relation Extraction

Jianfeng Qu (filin University), Wen Hua (University of Queensland),

Dantong Ouyang (filin University), Xiaofang Zhou (University of Queensland), Ximing Li (filin University)

- Learning Region Similarity over Spatial Knowledge Graphs with Hierarchical Types and Semantic Relations

Xiongnan Jin, Byungkook Oh, Sanghak Lee, Dongho Lee, Kyong-Ho Lee (Yonsei University),

Liang Chen (Sun Yat-sen University)

- Bayes EMbedding (BEM): Refining Representation by Integrating Knowledge Graphs and Behavior-specific Networks

Yuting Ye (University of California, Berkeley), Xuwu Wang (Fudan University),

Jiangchao Yao, Kunyang Jia, Jingren Zhou (Alibaba Group), Yanghua Xiao (Fudan University),

Hongxia Yang (Alibaba Group)

- A Benchmark for Fact Checking Algorithms Built on Knowledge Bases

Viet-Phi Huynh, Paolo Papotti (EURECOM)

\section{Session: Long - Knowledge Graph II}

- Online Schemaless Querying of Heterogeneous Open Knowledge Bases 699

Nikita Bhutani, H V Jagadish (University of Michigan)

- Enhancing Conversational Dialogue Models with Grounded Knowledge

Wen Zheng (University of Nottingham), Ke Zhou (University of Nottingham \& Nokia Bell Labs)

- MedTruth: A Semi-supervised Approach to Discovering Knowledge Condition

Information from Multi-Source Medical Data 719

Yang Deng (Peking University, Peng Cheng Laboratory, \& The Chinese University of Hong Kong),

Yaliang Li (Alibaba Group), Ying Shen (Peking University Shenzhen Graduate School),

Nan Du, Wei Fan (Tencent Medical AI Lab), Min Yang (Chinese Academy of Sciences),

Kai Lei (Peking University \& Peng Cheng Laboratory) 
- Look before you Hop: Conversational Question Answering over Knowledge Graphs Using Judicious Context Expansion

Philipp Christmann, Rishiraj Saha Roy, Abdalghani Abujabal, Jyotsna Singh,

Gerhard Weikum (MPI for Informatics)

- Learning to Answer Complex Questions over Knowledge Bases with Query Composition ... 739 Nikita Bhutani, Xinyi Zheng, H V Jagadish (University of Michigan)

\section{Session: Long - Machine Learning Themes I}

- Auto-completion for Data Cells in Relational Tables 761 Shuo Zhang, Krisztian Balog (University of Stavanger)

- Author Set Identification via Quasi-Clique Discovery . 771 Yuyan Zheng, Chuan Shi (Beijing University of Posts and Telecommunications), Xiangnan Kong (Worcester Polytechnic Institute), Yanfang Ye (Case Western Reserve University)

- AdaFair: Cumulative Fairness Adaptive Boosting 781 Vasileios Iosifidis, Eirini Ntoutsi (L3S Research Center, Leibniz University of Hannover)

- New Online Kernel Ridge Regression via Incremental Predictive Sampling 791 Shan Xu, Xiao Zhang, Shizhong Liao (Tianjin University)

- Online Kernel Selection via Tensor Sketching Shizhong Liao, Xiao Zhang (Tianjin University)

\section{Session: Long - Machine Learning Themes II}

- One-Class Active Learning for Outlier Detection with Multiple Subspaces

Holger Trittenbach, Klemens Böhm (Karlsruhe Institute of Technology (KIT))

- AutoGRD: Model Recommendation Through Graphical Dataset Representation 821 Noy Cohen-Shapira, Lior Rokach, Bracha Shapira, Gilad Katz, Roman Vainshtein (Ben Gurion University of the Negev)

- Batch Mode Active Learning for Semantic Segmentation Based on Multi-Clue Sample Selection...

Yao Tan, Liu Yang, Qinghua Hu (Tianjin University),

Zhibin Du (China Automotive Technology \& Research Center)

- CRUX: Adaptive Querying for Efficient Crowdsourced Data Extraction

Theodoros Rekatsinas (University of Wisconsin - Madison), Amol Deshpande (University of Maryland),

Aditya Parameswaran (University of California-Berkeley)

- Deep Forest with LRRS Feature for Fine-grained Website Fingerprinting with Encrypted SSL/TLS

Ziqing Zhang, Cuicui Kang, Gang Xiong, Zhen Li (Institute of Information Engineering,

Chinese Academy of Sciences \& University of Chinese Academy of Sciences)

\section{Session: Long - Machine Learning Themes III}

- N2N: Network Derivative Mining

Jian Kang, Hanghang Tong (University of Illinois at Urbana-Champaign)

- MoBoost: A Self-improvement Framework for Linear-based Hashing 871 Xingbo Liu (Shandong University), Xiushan Nie, Xiaoming Xi (Shandong fianzhu University), Lei Zhu (Shandong Normal University), Yilong Yin (Shandong University)

- Loopless Semi-Stochastic Gradient Descent with Less Hard Thresholding for Sparse Learning. 881 Xiangyang Liu, Bingkun Wei, Fanhua Shang, Hongying Liu (Xidian University)

- EPA: Exoneration and Prominence based Age for Infection Source Identification 891 Syed Shafat Ali (Jamia Millia Islamia), Tarique Anwar (Indian Institute of Technology Ropar), Ajay Rastogi, Syed Afzal Murtaza Rizvi (Famia Millia Islamia) 


\section{Session: Long - Mining in Emerging Applications I}

- Generating Persuasive Visual Storylines for Promotional Videos

Chang Liu, Yi Dong, Han Yu, Zhiqi Shen (Nanyang Technological University),

Zhanning Gao, Pan Wang, Changgong Zhang, Peiran Ren, Xuansong Xie (Alibaba Group),

Lizhen Cui (Shandong University), Chunyan Miao (Nanyang Technological University)

- Clustering Recurrent and Semantically Cohesive Program Statements

in Introductory Programming Assignments

Victor J. Marin, Carlos R. Rivero (Rochester Institute of Technology)

- Going Beyond Content Richness: Verified Information Aware Summarization

of Crisis-Related Microblogs

Ashish Sharma (University of Washington), Koustav Rudra (Leibniz Universität Hannover),

Niloy Ganguly (Indian Institute of Technology Kharagpur)

- Declarative User Selection with Soft Constraints

Yael Amsterdamer (Bar-Ilan University), Tova Milo, Amit Somech, Brit Youngmann (Tel Aviv University)

- \#suicidal - A Multipronged Approach to Identify and Explore Suicidal Ideation in Twitter ... 941 Pradyumna Prakhar Sinha, Rohan Mishra (Delhi Technological University),

Ramit Sawhney (Netaji Subhash Insitute of Technology), Debanjan Mahata (Bloomberg \& IIIT-Delhi),

Rajiv Ratn Shah (IIIT-Delhi), Huan Liu (Arizona State University)

\section{Session: Long - Mining in Emerging Applications II}

- MusicBot: Evaluating Critiquing-Based Music Recommenders with Conversational Interaction 951

Yucheng Jin (Lenovo Research), Wanling Cai (Hong Kong Baptist University),

Li Chen (Hong Kong Baptist University), Nyi Nyi Htun, Katrien Verbert (KU Leuven)

- Discovering Polarized Communities in Signed Networks. 961

Francesco Bonchi (ISI Foundation \& Eurecat), Edoardo Galimberti (University of Turin \& ISI Foundation), Aristides Gionis, Bruno Ordozgoiti (Aalto University), Giancarlo Ruffo (University of Turin)

- Model-based Constrained MDP for Budget Allocation in Sequential Incentive Marketing .... 971 Shuai Xiao, Le Guo, Zaifan Jiang, Lei Lv, Yuanbo Chen, Jun Zhu, Shuang Yang (Ant Financial Services Group)

- Wide-Ranging Review Manipulation Attacks\& Model, Empirical Study, and Countermeasures Parisa Kaghazgaran, Majid Alfifi, James Caverlee (Texas A\&M University)

- Augment to Prevent: Short-Text Data Augmentation in Deep Learning for Hate-Speech Classification Georgios Rizos, Konstantin Hemker (Imperial College London), Björn Schuller (Imperial College London \& University of Augsburg)

\section{Session: Long - Natural Language Processing I}

- Nested Relation Extraction with Iterative Neural Network

Yixuan Cao, Dian Chen, Hongwei Li, Ping Luo (Institute of Computing Technology, Chinese Academy of Sciences \& University of Chinese Academy of Sciences)

- Learning Chinese Word Embeddings from Stroke, Structure and Pinyin of Characters ........ 1011 Yun Zhang, Yongguo Liu, Jiajing Zhu, Ziqiang Zheng (University of Electronic Science and Technology of China), Xiaofeng Liu, Weiguang Wang, Zijie Chen, Shuangqing Zhai (Beijing University of Chinese Medicine)

- Sentiment Commonsense Induced Sequential Neural Networks for Sentiment Classification Chen Shiyun, Lin Xin (East China Normal University), Xiao Yanghua (Fudan University), He Liang (East China Normal University)

- Interactive Multi-Grained Joint Model for Targeted Sentiment Analysis 1031 Da Yin, Xiao Liu, Xiaojun Wan (Peking University)

- Beyond word2vec: Distance-graph Tensor Factorization for Word and Document Embeddings. 1041 Suhang Wang (The Pennsylvania State University), Charu Aggarwal (IBM T.F. Watson Research Center), Huan Liu (Arizona State University) 


\section{Session: Long - Natural Language Processing II}

- Hierarchical Multi-label Text Classification: An Attention-based Recurrent Network Approach 1051

Wei Huang, Enhong Chen, Qi Liu (University of Science and Technology of China), Yuying Chen (University of Science and Technology of China \& Ant Financial Services Group), Zai Huang, Yang Liu (University of Science and Technology of China), Zhou Zhao (Zhejiang University), Dan Zhang (iFLYTEK Research), Shijin Wang (iFLYTEK Research)

- A Semantics Aware Random Forest for Text Classification 1061 Md Zahidul Islam, Jixue Liu, Jiuyong Li, Lin Liu (University of South Australia), Wei Kang (Data61, CSIRO)

- Federated Topic Modeling.

Di Jiang, Yuanfeng Song (WeBank Co., Ltd), Yongxin Tong (Beihang University),

Xueyang $\mathrm{Wu}$ (The Hong Kong University of Science and Technology),

Weiwei Zhao, Qian Xu, Qiang Yang (WeBank Co., Ltd)

- Multi-Turn Response Selection in Retrieval-Based Chatbots with Iterated Attentive Convolution Matching Network.

Heyuan Wang, Ziyi Wu, Junyu Chen (Peking University)

- Sentiment Lexicon Enhanced Neural Sentiment Classification 1091 Chuhan Wu (Tsinghua University), Fangzhao Wu (Microsoft Research Asia), Junxin Liu, Yongfeng Huang (Tsinghua University), Xing Xie (Microsoft Research Asia)

\section{Session: Long - Deep Nerual Network I}

- ResumeGAN: An Optimized Deep Representation Learning Framework for Talent-Job Fit via Adversarial Learning 1101 Yong Luo, Huaizheng Zhang, Yonggang Wen (Nanyang Technological University), Xinwen Zhang (Hiretual)

- Regularizing Deep Neural Networks by Ensemble-based Low-Level Sample-Variances Method Shuai Yao, Yuexian Hou, Liangzhu Ge, Zeting Hu (Tianjin University)

- Attention-Residual Network with CNN for Rumor Detection 1121 Yixuan Chen, Jie Sui, Liang Hu, Wei Gong (University of Chinese Academy of Sciences)

- Imbalance Rectification in Deep Logistic Regression for Multi-Label Image Classification Using Random Noise Samples

Wenjin Yan, Ruixuan Li (Huazhong University of Science and Technology),

Jun Wang (Fujitsu Laboratories of America),

Yuhua Li, Jinyang Wang, Pan Zhou, Xiwu Gu (Huazhong University of Science and Technology)

- CamDrop: A New Explanation of Dropout and A Guided Regularization Method for Deep Neural Networks Hongjun Wang, Guangrun Wang, Guanbin Li, Liang Lin (Sun Yat-sen University)

\section{Session: Long - Deep Nerual Network II}

- Dynamic Collaborative Recurrent Learning

Teng Xiao (Sun Yat-sen University Sun Yat-sen University \& Guangdong Key Laboratory of Big Data Analysis and Processing), Shangsong Liang (Sun Yat-sen University \& Guangdong Key Laboratory of Big Data Analysis and Processing), Zaiqiao Meng (University of Glasgow)

- Autolnt: Automatic Feature Interaction Learning via Self-Attentive Neural Networks. 1161 Weiping Song, Chence Shi (Peking University), Zhiping Xiao (University of California, Los Angeles), Zhijian Duan, Yewen Xu, Ming Zhang (Peking University), Jian Tang (Mila-Quebec AI Institute, HEC Montreal \& CIFAR AI Chair)

- Automatic Construction of Multi-layer Perceptron Network from Streaming Examples 1171 Mahardhika Pratama (Nanyang Technological University), Choiru Za'in (La Trobe University), Andri Ashfahani, Yew Soon Ong (Nanyang Technological University), Weiping Ding (Nantong University)

- Robust Embedded Deep K-means Clustering 1181 Rui Zhang (Arizona State University), Hanghang Tong (University of Illinois at Urbana-Champaign \& Arizona State University), Yinglong Xia (Facebook), Yada Zhu (IBM T.F. Watson Research) 


\section{Session: Long - Network Science}

- Discovering Interesting Cycles in Directed Graphs

Florian Adriaens (Ghent University), Cigdem Aslay (Aalto University),

Tijl De Bie (Ghent University), Aristides Gionis (Aalto University), Jefrey Lijffijt (Ghent University)

- FLEET: Butterfly Estimation from a Bipartite Graph Stream.

Seyed-Vahid Sanei-Mehri, Yu Zhang (Iowa State University), Ahmet Erdem Sariyüce (University at Buffalo),

Srikanta Tirthapura (Iowa State University)

- Selecting the Optimal Groups: Efficiently Computing Skyline k-Cliques Chen Zhang, Wenjie Zhang (University of New South Wales),

Ying Zhang (University of Technology, Sydney), Lu Qin (University of Technology, Sydney),

Fan Zhang (Guangzhou University), Xuemin Lin (University of New South Wales)

- Balance in Signed Bipartite Networks.

Tyler Derr (Michigan State University), Cassidy Johnson (University of the Pacific),

Yi Chang (filin University), Jiliang Tang (Michigan State University)

- Adaptive Algorithms for Estimating Betweenness and $k$-path Centralities Mostafa Haghir Chehreghani (Amirkabir University of Technology (Tehran Polytechnic) \& Télécom-Paris, Institut Polytechnique de Paris (IP-Paris)), Albert Bifet, Talel Abdessalem (Télécom-Paris, IP-Paris)

\section{Session: Long - Online and Real-Time}

- Interactive Variance Attention based Online Spoiler Detection for Time-Sync Comments.

Wenmian Yang (Shanghai fiao Tong University), Weijia Jia (University of Macau),

Wenyuan Gao, Xiaojie Zhou, Yutao Luo (Shanghai fiao Tong University)

- Detecting Malicious Accounts in Online Developer Communities Using Deep Learning ..... 1251 Qingyuan Gong, Jiayun Zhang, Yang Chen (Fudan University),

Qi Li (Tsinghua University), Yu Xiao (Aalto University), Xin Wang (Fudan University),

Pan Hui (University of Helsinki \& Hong Kong University of Science and Technology)

- Exploring Multi-Objective Exercise Recommendations in Online Education Systems

Zhenya Huang, Qi Liu (University of Science and Technology of China),

Chengxiang Zhai (University of Illinois at Urbana-Champaign),

Yu Yin (University of Science and Technology of China),

Enhong Chen, Weibo Gao (University of Science and Technology of China), Guoping Hu (iFLYTEK Co., LTD)

- Into the Battlefield: Quantifying and Modeling Intra-community Conflicts in Online Discussion.

Subhabrata Dutta (Fadavpur University),

Gunkirat Kaur, Shreyans Mogia, Arpan Mukherjee (Indraprastha Institute of Information Technology),

Dipankar Das (Fadavpur University), Tanmoy Chakraborty (Indraprastha Institute of Information Technology)

- Offline and Online Satisfaction Prediction in Open-Domain Conversational Systems. 1281 Jason Ingyu Choi, Ali Ahmadvand, Eugene Agichtein (Emory University)

\section{Session: Long - Privacy}

- Privacy-Preserving Tensor Factorization for Collaborative Health Data Analysis

Jing Ma, Qiuchen Zhang, Jian Lou, Joyce C. Ho, Li Xiong (Emory University),

Xiaoqian Jiang (UT Health Science Center at Houston)

- Achieve Privacy-Preserving Truth Discovery in Crowdsensing Systems

Jianchao Tang (National University of Defense Technology),

ShaoJing Fu (National University of Defense Technology \& Sate Key Laboratory of Cryptology),

Ming Xu, Yuchuan Luo, Kai Huang (National University of Defense Technology)

- Privacy-preserving Crowd-guided AI Decision-making in Ethical Dilemmas

Teng Wang (Xi'an fiaotong University), Jun Zhao, Han Yu (Nanyang Technological University), Jinyan Liu (The University of Hong Kong), Xinyu Yang, Xuebin Ren (Xi'an fiaotong University), Shuyu Shi (Nanjing University) 
- Privacy Preserving Approximate K-means Clustering .

Chandan Biswas (Indian Statistical Institute), Debasis Ganguly (IBM Research Lab),

Dwaipayan Roy (GESIS - Leibniz Institute for the Social Sciences \& Indian Statistical Institute),

Ujjwal Bhattacharya (Indian Statistical Institute)

- Practical Access Pattern Privacy by Combining PIR and Oblivious Shuffle

Zhilin Zhang (Simon Fraser University \& Amazon), Ke Wang, Weipeng Lin (Simon Fraser University),

Ada Wai-Chee Fu (Chinese University of Hong Kong),

Raymond Chi-Wing Wong (Hong Kong University of Science and Technology)

\section{Session: Long - Question Answering and Dialogue Systems I}

- A Hybrid Retrieval-Generation Neural Conversation Model

Liu Yang (University of Massachusetts, Amherst), Junjie Hu (Carnegie Mellon University),

Minghui Qiu (Alibaba Group), Chen Qu (University of Massachusetts, Amherst),

Jianfeng Gao (Microsoft Research Redmond), W. Bruce Croft (University of Massachusetts, Amherst),

Xiaodong Liu (Microsoft Research Redmond), Yelong Shen (Tencent AI Lab),

Jingjing Liu (Microsoft Research Redmond)

- A Latent-Constrained Variational Neural Dialogue Model for Information-Rich Responses.

Yanan Zheng, Yan Wang (Central University of Finance and Economics),

Lijie Wen, Jianmin Wang (Tsinghua University)

- Legal Summarization for Multi-role Debate Dialogue via Controversy Focus Mining and Multi-task Learning.

Xinyu Duan (Zhejiang University), Yating Zhang (Alibaba Group), Lin Yuan (Zhejiang University),

Xin Zhou (Alibaba Group), Xiaozhong Liu (Indiana University Bloomington),

Tianyi Wang (Alibaba Group), Ruocheng Wang (Zhejiang University),

Qiong Zhang, Changlong Sun (Alibaba Group), Fei Wu (Zhejiang University)

- ConCET: Entity-Aware Topic Classification for Open-Domain Conversational Agents

Ali Ahmadvand, Harshita Sahijwani, Jason Ingyu Choi, Eugene Agichtein (Emory University)

- An Interactive Mechanism to Improve Question Answering Systems via Feedback.

Xinbo Zhang, Lei Zou, Sen Hu (Peking University)

\section{Session: Long - Question Answering and Dialogue Systems II}

- Attentive History Selection for Conversational Question Answering 1391

Chen Qu, Liu Yang (University of Massachusetts, Amherst), Minghui Qiu (Alibaba Group),

Yongfeng Zhang (Rutgers University), Cen Chen (Ant Financial Services Group),

W. Bruce Croft, Mohit Iyyer (University of Massachusetts, Amherst)

- Emotion-aware Chat Machine: Automatic Emotional Response Generation

for Human-like Emotional Interaction

Wei Wei, Jiayi Liu (Huazhong University of Science and Technology),

Xianling Mao (Beijing Institute of Technology), Guibing Guo (Northeastern University),

Feida Zhu (Singapore Management University),

Pan Zhou, Yuchong Hu (Huazhong University of Science and Technology)

- Commonsense Properties from Query Logs and Question Answering Forums

Julien Romero (Télécom Paris), Simon Razniewski, Koninika Pal (Max Planck Institute for Informatics), Jeff Z. Pan (University of Aberdeen), Archit Sakhadeo, Gerhard Weikum (Max Planck Institute for Informatics)

- Adapting Visual Question Answering Models for Enhancing Multimodal Community Q\&A Platforms

Avikalp Srivastava (Carnegie Mellon University), Hsin-Wen Liu (Waseda University),

Sumio Fujita (Yahoo! fapan)

- Message Passing for Complex Question Answering over Knowledge Graphs

Svitlana Vakulenko, Javier David Fernandez Garcia, Axel Polleres (Vienna University of Economics and Business), Maarten de Rijke (University of Amsterdam), Michael Cochez (Fraunhofer FIT) 


\section{Session: Long - Recommendation System I}

- BERT4Rec: Sequential Recommendation with Bidirectional Encoder Representations from Transformer

Fei Sun, Jun Liu, Jian Wu, Changhua Pei, Xiao Lin, Wenwu Ou, Peng Jiang (Alibaba Group)

- Adaptive Feature Sampling for Recommendation with Missing Content Feature Values Shaoyun Shi, Min Zhang (Tsinghua University), Xinxing Yu (Zhihu), Yongfeng Zhang (Rutgers University), Bin Hao, Yiqun Liu, Shaoping Ma (Tsinghua University)

- A Dynamic Co-attention Network for Session-based Recommendation 1461 Wanyu Chen (National University of Defense Technology \& University of Amsterdam), Fei Cai, Honghui Chen (National University of Defense Technology), Maarten de Rijke (University of Amsterdam)

- Attributed Multi-Relational Attention Network for Fact-checking URL Recommendation .......1471 Di You, Nguyen Vo, Kyumin Lee (Worcester Polytechnic Institute), Qiang LIU (Alibaba Group)

- A Hierarchical Self-Attentive Model for Recommending User-Generated Item Lists. 1481 Yun He, Jianling Wang, Wei Niu, James Caverlee (Texas A\&M University)

\section{Session: Long - Recommendation System II}

- HAES: A New Hybrid Approach for Movie Recommendation with Elastic Serendipity 1503 Xueqi Li, Wenjun Jiang, Weiguang Chen (Hunan University), Jie Wu (Temple University), Guojun Wang (Guangzhou University)

- DBRec: Dual-Bridging Recommendation via Discovering Latent Groups 1513 Jingwei Ma (University of Queensland), Jiahui Wen (National University of Defense Technology), Mingyang Zhong (Central Queensland University \& Chongqing Meiqi Industry Co.), Liangchen Liu (University of Queensland), Chaojie Li (Alibaba Group), Weitong Chen (University of Queensland), Yin Yang (Hamad Bin Khalifa University), Hongkui Tu (National University of Defense Technology), Xue Li (University of Queensland)

- Candidate Generation with Binary Codes for Large-Scale Top-N Recommendation 1523 Wang-Cheng Kang, Julian McAuley (University of California, San Diego)

- DTCDR: A Framework for Dual-Target Cross-Domain Recommendation Feng Zhu (Macquarie University), Chaochao Chen (Ant Financial Services Group), Yan Wang, Guanfeng Liu (Macquarie University), Xiaolin Zheng (Zhejiang University)

- Recommender System Using Sequential and Global Preference via Attention Mechanism and Topic Modeling 1543 Kyeongpil Kang (Korea University \& SKT AI Center), Junwoo Park (SK Planet), Wooyoung Kim (Korea University \& SKT AI Center), Hojung Choe (NICE Information Service), Jaegul Choo (Korea University)

\section{Session: Long - Recommendation System III}

- A Spatio-temporal Recommender System for On-demand Cinemas Taofeng Xue, Beihong Jin, Beibei Li (Institute of Software, Chinese Academy of Sciences \& University of Chinese Academy of Sciences), Weiqing Wang (Monash University), Qi Zhang, Sihua Tian (Beijing iQIYI Cinema Management Co., Ltd.)

- Semi-Supervised Learning for Cross-Domain Recommendation to Cold-Start Users 1563 SeongKu Kang, Junyoung Hwang, Dongha Lee, Hwanjo Yu (POSTECH)

- Leveraging Ratings and Reviews with Gating Mechanism for Recommendation 1573 Haifeng Xia, Zengmao Wang, Bo Du, Lefei Zhang (Wuhan University), Shuai Chen, Gang Chun (Xiaomi Inc.)

- Instagrammers, Fashionistas, and Me: Recurrent Fashion Recommendation with Implicit Visual Influence. 1583 Yin Zhang, James Caverlee (Texas A\&M University)

- What Can History Tell Us? Identifying Relevant Sessions for Next-Item Recommendation 1593 Ke Sun, Tieyun Qian (Wuhan University), Hongzhi Yin, Tong Chen (University of Queensland), Yiqi Chen (Wuhan University), Ling Chen (University of Technology Sydney) 


\section{Session: Long - Reinforcement Learning}

- Context-Aware Ranking by Constructing a Virtual Environment for Reinforcement Learning.

Junqi Zhang, Jiaxin Mao, Yiqun Liu, Ruizhe Zhang, Min Zhang, Shaoping Ma (Tsinghua University), Jun Xu (Renmin University of China), Qi Tian (Huawei Noah's Ark Lab)

- A Multi-Scale Temporal Feature Aggregation Convolutional Neural Network for Portfolio Management 1613 Si Shi, Jianjun Li, Guohui Li, Peng Pan (Huazhong University of Science and Technology)

- Order-free Medicine Combination Prediction with Graph Convolutional Reinforcement Learning..... Shanshan Wang (Shandong University), Pengjie Ren (University of Amsterdam), Zhumin Chen, Zhaochun Ren, Jun Ma (Shandong University), Maarten de Rijke (University of Amsterdam)

- Reinforcement Learning with Sequential Information Clustering in Real-Time Bidding ..... 1633 Junwei Lu, Chaoqi Yang, Xiaofeng Gao (Shanghai fiao Tong University), Liubin Wang, Changcheng Li (Tencent), Guihai Chen (Shanghai fiao Tong University)

- Generative Question Refinement with Deep Reinforcement Learning in Retrieval-based QA System.

Ye Liu (University of Illinois at Chicago), Chenwei Zhang (Amazon), Xiaohui Yan (Poisson Lab, Huawei Technologies), Yi Chang (filin University), Philip S. Yu (University of Illinois at Chicago)

\section{Session: Long - Search \& Retrieval}

- Analyzing the Effects of Document's Opinion and Credibility on Search Behaviors and Belief Dynamics

Suppanut Pothirattanachaikul (Kyoto University), Takehiro Yamamoto (University of Hyogo),

Yusuke Yamamoto (Shizuoka University), Masatoshi Yoshikawa (Kyoto University)

- Identifying Facet Mismatches In Search Via Micrographs 1663 Sriram Srinivasan (University of California, Santa Cruz), Nikhil S. Rao, Karthik Subbian (Amazon Inc.), Lise Getoor (University of California, Santa Cruz)

- GRIP: Multi-Store Capacity-Optimized High-Performance Nearest Neighbor Search for Vector Search Engine 1673 Minjia Zhang, Yuxiong He (Microsoft AI and Research)

- Improving Web Image Search with Contextual Information 1683 Xiaohui Xie, Jiaxin Mao, Yiqun Liu (Tsinghua University), Maarten de Rijke (University of Amsterdam), Qingyao Ai (University of Utah), Yufei Huang, Min Zhang, Shaoping Ma (Tsinghua Unviersity)

- Dynamic Bayesian Metric Learning for Personalized Product Search Teng Xiao, Jiaxin Ren (Sun Yat-sen University), Zaiqiao Meng (University of Glasgow), Huan Sun (The Ohio State University), Shangsong Liang (Sun Yat-sen University)

\section{Session: Long - Sequential Data Analysis}

- Towards Accurate and Interpretable Sequential Prediction: A CNN \& Attention-Based Feature Extractor

Jingyi Wang (University of Electronic Science and Technology of China \& RealAI),

Qiang Liu (RealAI and Tsinghua University), Zhaocheng Liu (RealAI), Shu Wu (Chinese Academy of Sciences)

- Locally Slope-based Dynamic Time Warping for Time Series Classification. Jidong Yuan, Qianhong Lin, Wei Zhang, Zhihai Wang (Beijing fiaotong University)

- HiCAN: Hierarchical Convolutional Attention Network for Sequence Modeling 1723 Yi Cao, Weifeng Zhang, Bo Song, Congfu Xu (Zhejiang University)

- Automatic Sequential Pattern Mining in Data Streams Koki Kawabata, Yasuko Matsubara, Yasushi Sakurai (ISIR, Osaka University)

- Efficient Sequential and Parallel Algorithms for Estimating Higher Order Spectra 1743 Zigeng Wang (University of Connecticut), Abdullah-Al Mamun (University of Connecticut \& Google LLC), Xingyu Cai, Nalini Ravishanker, Sanguthevar Rajasekaran (University of Connecticut) 


\section{Session: Long - Social Network}

- A Modular Adversarial Approach to Social Recommendation

Adit Krishnan, Hari Cheruvu, Cheng Tao, Hari Sundaram (University of Illinois at Urbana-Champaign)

- Emotional Contagion-Based Social Sentiment Mining in Social Networks by Introducing Network Communities

Xiaobao Wang, Di Jin, Mengquan Liu, Dongxiao He (Tianjin University),

Katarzyna Musial (University of Technology Sydney),

Jianwu Dang (Japan Advanced Institute of Science and Technology)

- Social-Aware VR Configuration Recommendation via Multi-Feedback Coupled Tensor Factorization Hsu-Chao Lai (National Chiao Tung University \& Academia Sinica),

Hong-Han Shuai (National Chiao Tung University), De-Nian Yang (Academia Sinica),

Jiun-Long Huang (National Chiao Tung University), Wang-Chien Lee (The Pennsylvania State University), Philip S. Yu (University of Illinois at Chicago \& Fudan University)

- Tracking Top-k Influential Users with Relative Errors. 1783

Yu Yang (City University of Hong Kong), Zhefeng Wang (Huawei Tech.),

Tianyuan Jin (University of Science and Technology of China), Jian Pei (Simon Fraser University),

Enhong Chen (University of Science and Technology of China)

- NActSeer: Predicting User Actions in Social Network using Graph

Augmented Neural Network

Mohammad Raihanul Islam, Sathappan Muthiah, Naren Ramakrishnan (Virginia Tech)

\section{Session: Long - Understanding and Interpretability I}

- In2Rec: Influence-based Interpretable Recommendation

Huafeng Liu, Jingxuan Wen, Liping Jing, Jian Yu (Beijing fiaotong University),

Xiangliang Zhang (King Abdullah University of Science and Technology), Min Zhang (Tsinghua University)

- Accounting for Temporal Dynamics in Document Streams

Zhendong Chu (Fudan University), Renqin Cai, Hongning Wang (University of Virginia)

- How Does BERT Answer Questions? A Layer-Wise Analysis of Transformer Representations

Betty van Aken, Benjamin Winter, Alexander Löser, Felix A. Gers (Beuth University of Applied Sciences)

- Patterns of Search Result Examination: Query to First Action Mustafa Abualsaud, Mark D. Smucker (University of Waterloo)

- A Dynamic Product-aware Learning Model for E-commerce Query Intent Understanding . 1843 Jiashu Zhao (Wilfrid Laurier University), Hongshen Chen, Dawei Yin (FD.COM)

\section{Session: Long - Understanding and Interpretability II}

- Scalable Causal Graph Learning through a Deep Neural Network 1853 Chenxiao Xu (Stony Brook University), Hao Huang (GE Global Research), Shinjae Yoo (Stony Brook University)

- Interpretable Multiple-Kernel Prototype Learning for Discriminative Representation and Feature Selection 1863 Babak Hosseini, Barbara Hammer (CITEC Cluster of Excellence, Bielefeld University)

- BePT: A Behavior-based Process Translator for Interpreting and Understanding Process Models Chen Qian, Lijie Wen (Tsinghua University), Akhil Kumar (Penn State University)

- Towards Effective and Interpretable Person-Job Fitting 1883 Ran Le, Wenpeng Hu (Peking University), Yang Song (BOSS Zhipin NLP Center), Tao Zhang (BOSS Zhipin), Dongyan Zhao, Rui Yan (Peking University)

- Leveraging Graph Neighborhoods for Efficient Inference. 1893 Melisachew Wudage Chekol, Heiner Stuckenschmidt (University of Mannheim) 


\section{Session: Long - Urban Computing I}

- STAR: Spatio-Temporal Taxonomy-Aware Tag Recommendation for Citizen Complaints... 1903 Jingyue Gao, Yuanduo He, Yasha Wang (Peking Univeristy),

Xiting Wang (Microsoft Research Asia), Jiangtao Wang (Lancaster University),

Guangju Peng, Xu Chu (Peking Univeristy)

- CoLight: Learning Network-level Cooperation for Traffic Signal Control

Hua Wei (The Pennsylvania State University), Nan Xu, Huichu Zhang (Shanghai fiao Tong University),

Guanjie Zheng (The Pennsylvania State University),

Xinshi Zang, Chacha Chen, Weinan Zhang, Yanmin Zhu (Shanghai fiao Tong University),

Kai Xu (Shanghai Tianrang Intelligent Technology Co., Ltd), Zhenhui Li (The Pennsylvania State University)

- Learning to Effectively Estimate the Travel Time for Fastest Route Recommendation 1923 Ning Wu, Jingyuan Wang (Beihang University), Wayne Xin Zhao (Renmin University of China), Yang Jin (Beihang University)

- PRNet: Outdoor Position Recovery for Heterogenous Telco Data by Deep Neural Network.

Yige Zhang, Weixiong Rao (Tongii University), Kun Zhang (Carnegie Mellon University),

Mingxuan Yuan, Jia Zeng (Huawei Noah's Ark Lab)

- Active Collaborative Sensing for Energy Breakdown

Yiling Jia (University of Virginia), Nipun Batra (IIT Gandhinagar),

Hongning Wang, Kamin Whitehouse (University of Virginia)

\section{Session: Long - Urban Computing II}

- Forecasting Pavement Performance with a Feature Fusion LSTM-BPNN Model.

Yushun Dong, Yingxia Shao, Xiaotong Li (Beijing University of Posts and Telecommunications),

Sili Li, Lei Quan (Research Institute of Highway, Ministry of Transportation),

Wei Zhang (East China Normal University),

Junping Du (Beijing University of Posts and Telecommunications)

- Learning Phase Competition for Traffic Signal Control

Guanjie Zheng (The Pennsylvania State University), Yuanhao Xiong (Zhejiang Univerisity),

Xinshi Zang (Shanghai fiao Tong Univerisity), Jie Feng (Tsinghua Univerisity),

Hua Wei (The Pennsylvania State University), Huichu Zhang (Shanghai fiao Tong Univerisity),

Yong Li (Tsinghua Univerisity), Kai Xu (Shanghai Tianrang Intelligent Technology Co., Ltd),

Zhenhui Li (The Pennsylvania State University)

- Path Travel Time Estimation using Attribute-related Hybrid Trajectories Network 1973

Xi Lin (Nanyang Technological University), Yequan Wang (Tencent Inc.),

Xiaokui Xiao (National University of Singapore),

Zengxiang Li (Institute of High Performance Computing),

Sourav S. Bhowmick (Nanyang Technological University)

- CoRide: Joint Order Dispatching and Fleet Management for Multi-Scale Ride-Hailing Platforms 1983

Jiarui Jin, Ming Zhou, Weinan Zhang (Shanghai fiao Tong University),

Minne Li (University College London), Zilong Guo (Shanghai fiao Tong University),

Zhiwei Qin, Yan Jiao, Xiaocheng Tang, Chenxi Wang (DiDi AI Labs),

Jun Wang (University College London), Guobin Wu (DiDi Research), Jieping Ye (DiDi AI Labs)

- Unsupervised Representation Learning of Spatial Data via Multimodal Embedding 1993 Porter Jenkins (The Pennsylvania State University), Ahmad Farag (Georgia Tech University), Suhang Wang, Zhenhui Li (The Pennsylvania State University)

\section{Session: Long - User Behavior}

- Rating Mechanisms for Sustainability of Crowdsourcing Platforms 2003 Chenxi Qiu (Rowan University), Anna Squicciarini, Sarah Rajtmajer (The Pennsylvania State University)

- Exploring The Interaction Effects for Temporal Spatial Behavior Prediction 2013 Huan Yang, Tianyuan Liu, Yuqing Sun (Shandong University), Elisa Bertino (Purdue University)

- Social Cards Probably Provide For Better Understanding Of Web Archive Collections 2023 Shawn M. Jones, Michele C. Weigle, Michael L. Nelson (Old Dominion University) 
- Learning from Dynamic User Interaction Graphs to Forecast Diverse Social Behavior.

Prasha Shrestha, Suraj Maharjan, Dustin Arendt, Svitlana Volkova (Pacific Northwest National Laboratory)

- Understanding Default Behavior in Online Lending.

Yang Yang (Zhejiang University), Yuhong Xu (Netease Inc.), Chunping Wang (PPDAI Group Inc.),

Yizhou Sun (University of California, Los Angeles), Fei Wu, Yueting Zhuang (Zhejiang University),

Ming Gu (PPDAI Group Inc.)

\section{Session: Short - Interpretability \& Reasoning}

- Interpretable MTL from Heterogeneous Domains using Boosted Tree

Ya-Lin Zhang, Longfei Li (Ant Financial Services Group)

- Machine Reading Comprehension: Matching and Orders

Ao Liu (University of Electronic Science and Technology of China), Lizhen Qu (Monash University),

Junyu Lu, Chenbin Zhang, Zenglin Xu (University of Electronic Science and Technology of China)

- Aspect and Opinion Aware Abstractive Review Summarization

with Reinforced Hard Typed Decoder.

Yufei Tian (Tsinghua University), Jianfei Yu, Jing Jiang (Singapore Management University)

- Datalog Reasoning over Compressed RDF Knowledge Bases

Pan Hu (University of Oxford), Jacopo Urbani (Vrije Universiteit Amsterdam),

Boris Motik, Ian Horrocks (University of Oxford)

- An Explainable Deep Fusion Network for Affect Recognition

Using Physiological Signals

Jionghao Lin, Shirui Pan, Cheng Siong Lee, Sharon Oviatt (Monash University)

\section{Session: Short - Machine Learning}

- MarlRank: Multi-agent Reinforced Learning to Rank

Shihao Zou (University of Alberta \& University of College London \& Huawei),

Zhonghua Li (Huawei), Mohammad Akbari, Jun Wang (University College London),

Peng Zhang (Tianjin University)

- LinkRadar: Assisting the Analysis of Inter-app Page Links via Transfer Learning.

Diandian Gu (Peking University), Ziniu Hu (University of California, Los Angeles),

Shangchen Du (Peking University), Yun Ma (Tsinghua University)

- NAD: Neural Network Aided Design for Textile Pattern Generation.

Zhifei Pang, Sai Wu, Dongxiang Zhang, Yunjun Gao, Gang Chen (Zhejiang University)

- Feature Selection for Facebook Feed Ranking System via a Group-Sparsity-Regularized

Training Algorithm

Xiuyan Ni (City University of New York), Yang Yu, Peng Wu, Youlin Li (Facebook Inc.),

Shaoliang Nie (North Carolina State University), Qichao Que (Facebook Inc.), Chao Chen (Stony Brook University)

- Fine-Grained Geolocalization of User-Generated Short Text based on Weight Probability Model.

Congjie Gao, Yongjnu Li, Jiaqi Yang (Northwestern Polytechnical University)

- A Compare-Aggregate Model with Latent Clustering for Answer Selection Seunghyun Yoon (Seoul National University), Franck Dernoncourt, Doo Soon Kim, Trung Bui (Adobe Research), Kyomin Jung (Seoul National University)

\section{Session: Short - Embeddings}

- Spotting Terrorists by Learning Behavior-aware Heterogeneous Network Embedding. Pei-Chi Wang, Cheng-Te Li (National Cheng Kung University)

- Scalable Manifold-Regularized Attributed Network Embedding via Maximum Mean Discrepancy Jun Wu, Jingrui He (University of Illinois at Urbana-Champaign)

- Tensor Decomposition-based Node Embedding. 2105 Shah Muhammad Hamdi, Soukaïna Filali Boubrahimi, Rafal Angryk (Georgia State University) 
- Geometric Estimation of Specificity within Embedding Spaces

Negar Arabzadeh, Fattaneh Zarrinkalam (Ryerson University), Jelena Jovanovic (University of Belgrade),

Ebrahim Bagheri (Ryerson University)

- Similarity-Aware Network Embedding with Self-Paced Learning

Chao Huang (University of Notre Dame), Baoxu Shi (LinkedIn), Xuchao Zhang (Virginia Tech),

Xian $\mathrm{Wu}$ (University of Notre Dame), Nitesh V. Chawla (University of Notre Dame)

- Integrating Multi-Network Topology via Deep Semi-supervised Node Embedding. Hansheng Xue, Jiajie Peng (Northwestern Polytechnical University), Jiying Li (Microsoft Corporation), Xuequn Shang (Northwestern Polytechnical University)

\section{Session: Short - Time Sequences \& Dynamics}

- Query-Specific Knowledge Summarization with Entity Evolutionary Networks

Carl Yang, Lingrui Gan, Zongyi Wang, Jiaming Shen, Jinfeng Xiao,

Jiawei Han (University of Illinois at Urbana Champaign)

- Real-time Edge Repartitioning for Dynamic Graph. He Li, Hang Yuan, Jianbin Huang (Xidian University)

- DSANet: Dual Self-Attention Network for Multivariate Time Series Forecasting Siteng Huang, Donglin Wang (Westlake University), Xuehan Wu (Huawei Technologies Co., Ltd.), Ao Tang (WeCar Technology Co., Ltd.)

- Time Series Prediction with Interpretable Data Reconstruction Qiangxing Tian (Zhejiang University \& Westlake University), Jinxin Liu, Donglin Wang (Westlake University), Ao Tang (WeCar (Shenzhen) Technology Co., Ltd.)

- Towards Explainable Representation of Time-Evolving Graphs via Spatial-Temporal Graph Attention Networks

Zhining Liu (University of Electronic Science and Technology of China),

Dawei Zhou, Jingrui He (University of Illinois at Urbana-Champaign)

- Deep Prototypical Networks for Imbalanced Time Series Classification under Data Scarcity Chao Huang, Xian Wu (University of Notre Dame), Xuchao Zhang (Virginia Tech),

Suwen Lin, Nitesh V. Chawla (University of Notre Dame)

\section{Session: Short - Graph Neural Networks}

- Knowledge-aware Textual Entailment with Graph Attention Network

Daoyuan Chen (Peking University Shenzhen Graduate School), Yaliang Li (Alibaba Group),

Min Yang (Shenzhen Institutes of Advanced Technology, Chinese Academy of Sciences),

Hai-Tao Zheng (Tsinghua University), Ying Shen (Peking University Shenzhen Graduate School)

- Fast Approximations of Betweenness Centrality with Graph Neural Networks

Sunil Kumar Maurya (Tokyo Institute of Technology), Xin Liu (AIRC, AIST),

Tsuyoshi Murata (Tokyo Institute of Technology)

- Neighborhood Interaction Attention Network for Link Prediction

Zhitao Wang, Yu Lei, Wenjie Li (The Hong Kong Polytechnic University)

- Long-short Distance Aggregation Networks for Positive Unlabeled Graph Learning Man Wu (Florida Atlantic University), Shirui Pan, Lan Du (Monash University), Ivor Tsang (University of Technology Sydney), Xingquan Zhu (Florida Atlantic University), Bo Du (Wuhan University)

- Using External Knowledge for Financial Event Prediction Based on Graph Neural Networks

Yiying Yang (Fudan University \& Pingan Life Insurance),

Zhongyu Wei, Qin Chen, Libo Wu (Fudan University)

- Cross-Domain Recommendation via Preference Propagation GraphNet Cheng Zhao, Chenliang Li (Wuhan University), Cong Fu (Zhejiang University) 


\section{Session: Short - Recommendation}

- ARP: Aspect-aware Neural Review Rating Prediction

Chuhan Wu (Tsinghua University), Fangzhao Wu (Microsoft Research Asia),

Junxin Liu, Yongfeng Huang (Tsinghua University), Xing Xie (Microsoft Research Asia)

- CosRec: 2D Convolutional Neural Networks for Sequential Recommendation.

An Yan, Shuo Cheng, Wang-Cheng Kang, Mengting Wan, Julian McAuley (University of California, San Diego)

- Data Poisoning Attacks on Cross-domain Recommendation

Huiyuan Chen, Jing Li (Case Western Reserve University)

- Session-based Recommendation with Hierarchical Memory Networks Bo Song, Yi Cao, Weifeng Zhang, Congfu Xu (Zhejiang University)

- Correcting for Recency Bias in Job Recommendation 2185 Ruey-Cheng Chen (RMIT University), Qingyao Ai (University of Utah), Gaya Jayasinghe (GO1), W. Bruce Croft (RMIT University)

- Motif Enhanced Recommendation over Heterogeneous Information Network 2189 Huan Zhao, Yingqi Zhou, Yangqiu Song, Dik Lun Lee (Hong Kong University of Science and Technology)

\section{Session: Short - Algorithm}

- GPU-Accelerated Decoding of Integer Lists Antonio Mallia, Michał Siedlaczek, Torsten Suel, Mohamed Zahran (New York University)

- Synergizing Local and Global Models for Matrix Approximation Chao Chen (Shanghai fiao Tong University \& IBM Research China), Hao Zhang (Shanghai fiao Tong University), Dongsheng Li (IBM Research China), Junchi Yan, Xiaokang Yang (Shanghai fiao Tong University)

- Deep Colorization by Variation 2201 Zineng Tang (University of North Carolina at Chapel Hill)

- Fast Random Forest Algorithm via Incremental Upper Bound Yasuhiro Fujiwara (NTT Communication Science Laboratories), Yasutoshi Ida, Sekitoshi Kanai, Atsutoshi Kumagai, Junya Arai (NTT Software Innovation Center), Naonori Ueda (NTT Communication Science Laboratories)

- Convolution-Consistent Collective Matrix Completion 2209 Xu Liu (Arizona State University), Jingrui He (University of Illinois at Urbana-Champaign), Sam Duddy, Liz O’Sullivan (Allstate Northern Ireland)

- Faster Algorithms for $\boldsymbol{k}$-Regret Minimizing Sets via Monotonicity and Sampling. 2213 Qi Dong, Jiping Zheng (Nanjing University of Aeronautics and Astronautics)

- Towards Stochastic Simulations of Relevance Profiles 2217 Kevin Roitero, Andrea Brunello (University of Udine), Julián Urbano (Delft University of Technology), Stefano Mizzaro (University of Udine)

\section{Session: Short - Anomaly Detection}

- SpecAE: Spectral AutoEncoder for Anomaly Detection in Attributed Networks 2233 Yuening Li, Xiao Huang (Texas A\&M University), Jundong Li (University of Virginia), Mengnan Du, Na Zou (Texas A\&M University)

- On Continuously Matching of Evolving Graph Patterns Qianzhen Zhang, Deke Guo, Xiang Zhao, Aibo Guo (National University of Defense Technology)

- Time-Series Aware Precision and Recall for Anomaly Detection: Considering Variety of Detection Result and Addressing Ambiguous Labeling. Won-Seok Hwang, Jeong-Han Yun, Jonguk Kim, Hyoung Chun Kim (The Affiliated Institute of ETRI)

- Additive Explanations for Anomalies Detected from Multivariate Temporal Data 2245 Ioana Giurgiu, Anika Schumann (IBM Research - Zurich) 
- ED2: A Case for Active Learning in Error Detection

Felix Neutatz (DFKI GmbH), Mohammad Mahdavi (TU Berlin),

Ziawasch Abedjan (TU Berlin \& DFKI GmbH)

- Multi-scale Trajectory Clustering to Identify Corridors in Mobile Networks

Li Li, Sarah Erfani, Chien Aun Chan, Christopher Leckie (University of Melbourne)

\section{Session: Short - Recognition}

- Multi-view Moments Embedding Network for 3D Shape Recognition Jun Xiao, Yuanxing Zhang, Pengyu Zhao, Kecheng Xiao, Kaigui Bian, Chunli Zhang, Wei Yan (Peking University)

- Active Entity Recognition in Low Resource Settings Ning Gao, Nikos Karampatziakis, Rahul Potharaju (Microsoft), Silviu Cucerzan (Microsoft Research)

- On Novel Object Recognition: A Unified Framework for Discriminability and Adaptability Kai Li (Northeastern University), Martin Renqiang Min (NEC Labs America - Princeton), Bing Bai (NEC Labs America - Princeton), Yun Fu (Northeastern University), Hans Peter Graf (NEC Labs America - Princeton)

- Exploiting Multiple Embeddings for Chinese Named Entity Recognition Canwen Xu, Feiyang Wang (Wuhan University), Jialong Han (Tencent AI Lab), Chenliang Li (Wuhan University)

- Gate-based Bidirectional Interactive Decoding Network for Scene Text Recognition 2273 Yunze Gao, Yingying Chen, Jinqiao Wang, Hanqing Lu (Institute of Automation, Chinese Academy of Sciences)

- Modeling Long-Range Context for Concurrent Dialogue Acts Recognition 2277 Yue Yu, Siyao Peng, Grace Hui Yang (Georgetown University)

\section{Session: Short - Urbanism and Mobility}

- Labelling for Venue Visit Detection by Matching Wi-Fi Hotspots with Businesses 2281 Denis Shaposhnikov, Anastasia Bezzubtseva, Ekaterina Gladkikh, Alexey Drutsa (Yandex)

- Heterogeneous Components Fusion Network for Load Forecasting of Charging Stations ... 2285 Kai Li (independent), Fei Yu (Harbin Institute of Technology), Cheng Feng (Northeast Forestry University), Tian Xia (Harbin University of Science and Technology)

- Learning Traffic Signal Control from Demonstrations 2289 Yuanhao Xiong (Zhejiang University), Guanjie Zheng (The Pennsylvania State University), Kai Xu (Shanghai Tianrang Intelligent Technology Co., Ltd), Zhenhui Li (The Pennsylvania State University)

- Spatio-Temporal Graph Convolutional and Recurrent Networks for Citywide Passenger Demand Prediction .... 2293 Lei Bai, Lina Yao, Salil S. Kanhere (University of New South Wales), Xianzhi Wang (University of Technology Sydney), Wei Liu (University of New South Wales), Zheng Yang (Tsinghua University)

- Collaborative Analysis for Computational Risk in Urban Water Supply Systems 2297 Di Wu, Hao Wang, Razak Seidu (Norwegian University of Science and Technology)

- Long- and Short-term Preference Learning for Next POI Recommendation 2301 Yuxia Wu, Ke Li, Guoshuai Zhao, Xueming Qian (Xi'an fiaotong University)

\section{Session: Short - Information Retrieval}

- Cluster-Based Focused Retrieval 2305 Eilon Sheetrit, Oren Kurland (Technion - Israel Institute of Technology)

- Cross-modal Image-Text Retrieval with Multitask Learning 2309 Junyu Luo (SIAT, Chinese Academy of Sciences \& Sichuan University), Ying Shen (Peking University), Xiang Ao (ICT, Chinese Academy of Sciences), Zhou Zhao (Zhejiang University), Min Yang (SIAT, Chinese Academy of Sciences) 
- A Unified Generation-Retrieval Framework for Image Captioning....

Chunpu Xu (Huazhong University of Science and Technology),

Wei Zhao (Technische Universität Darmstadt), Min Yang (SIAT, Chinese Academy of Sciences),

Xiang Ao (ICT, Chinese Academy of Sciences),

Wangrong Cheng, Jinwen Tian (Huazhong University of Science and Technology)

- A Lossy Compression Method on Positional Index for Efficient and Effective Retrieval. Shuni Gao, Jipeng Liu, Xiaoguang Liu, Gang Wang (Nankai University)

- Interactive Matching Network for Multi-Turn Response Selection in Retrieval-Based Chatbots Jia-Chen Gu, Zhen-Hua Ling (University of Science and Technology of China), Quan Liu (University of Science and Technology of China \& iFLYTEK Research)

- Analysis of Adaptive Training for Learning to Rank in Information Retrieval Saar Kuzi, Sahiti Labhishetty (University of Illinois at Urbana-Champaign), Shubhra Kanti Karmaker Santu (Massachusetts Institute of Technology), Prasad Pradip Joshi (Unbxd), ChengXiang Zhai (University of Illinois at Urbana-Champaign)

\section{Session: Short - E-commerce \& Production}

- QPIN: A Quantum-inspired Preference Interactive Network for E-commerce Recommendation.

Panpan Wang (Tianjin University), Zhao Li (Alibaba Group),

Yazhou Zhang, Yuexian Hou, Liangzhu Ge (Tianjin University)

- A Study of Context Dependencies in Multi-page Product Search

Keping Bi (University of Massachusetts, Amherst),

Choon Hui Teo, Yesh Dattatreya, Vijai Mohan (Search Labs, Amazon),

W. Bruce Croft (University of Massachusetts, Amherst)

- Query-bag Matching with Mutual Coverage for Information-seeking

Conversations in E-commerce

Zhenxin Fu (Peking University \& Alibaba Group), Feng Ji (Alibaba Group), Wenpeng Hu (Peking University),

Wei Zhou (Alibaba Group), Dongyan Zhao (Peking University), Haiqing Chen (Alibaba Group),

Rui Yan (Peking University)

- Neural Review Rating Prediction with User and Product Memory

Zhigang Yuan (Tsinghua University), Fangzhao Wu (Microsoft Research Asia),

Junxin Liu, Chuhan Wu, Yongfeng Huang (Tsinghua University),

Xing Xie (Microsoft Research Asia)

- Intent Term Weighting in E-commerce Queries.

Saurav Manchanda (University of Minnesota), Mohit Sharma (WalmartLabs),

George Karypis (University of Minnesota)

- Fine-Grained Product Categorization in E-commerce

Hongshen Chen ( $(\mathcal{D} . C O M)$, Jiashu Zhao (Wilfrid Laurier University), Dawei Yin ( $(F D . C O M)$

\section{Session: Short - Classification}

- Large Margin Prototypical Network for Few-shot Relation Classification with Fine-grained Features Miao Fan, Yeqi Bai, Mingming Sun, Ping Li (Baidu Research)

- Meta-GNN: On Few-shot Node Classification in Graph Meta-learning

Fan Zhou, Chengtai Cao (University of Electronic Science and Technology of China), Kunpeng Zhang (University of Maryland), Goce Trajcevski (Iowa State University), Ting Zhong, Ji Geng (University of Electronic Science and Technology of China)

- Enriching Pre-trained Language Model with Entity Information for Relation Classification. Shanchan Wu, Yifan He (Alibaba Group (U.S.) Inc.)

- Unsupervised Concept Drift Detection with a Discriminative Classifier 2365 Ömer Gözüaçık, Alican Büyükçakır (Bilkent University), Hamed Bonab (University of Massachusetts, Amherst), Fazli Can (Bilkent University) 
- Hybrid Deep Pairwise Classification for Author Name Disambiguation.

Kunho Kim, Shaurya Rohatgi, C. Lee Giles (The Pennsylvania State University)

- Approximate Definitional Constructs as Lightweight Evidence for Detecting Classes Among Wikipedia Articles Marius Pasca (Google)

\section{Session: Short - Knowledge Extraction \& Generation}

- Towards the Gradient Vanishing, Divergence Mismatching and Mode Collapse of Generative Adversarial Nets

Zhaoyu Zhang, Changwei Luo, Jun Yu (University of Science and Technology of China)

- Generating Paraphrase with Topic as Prior Knowledge

Yuanxin Liu (Institute of Information Engineering, Chinese Academy of Sciences \& University of Chinese Academy of Sciences), Zheng Lin (Institute of Information Engineering, Chinese Academy of Sciences),

Fenglin Liu (Peking University), Qinyun Dai (Shanghai Branch, CNCERT/CC),

Weiping Wang (Institute of Information Engineering, Chinese Academy of Sciences)

- Sexual Harassment Story Classification and Key Information Identification Yingchi Liu, Quanzhi Li (Alibaba Group US), Xiaozhong Liu (Indiana University Bloomington), Qiong Zhang, Luo Si (Alibaba Group US)

- Neural Review Summarization Leveraging User and Product Information. Hui Liu, Xiaojun Wan (Peking University)

- Incorporating Relation Knowledge into Commonsense Reading Comprehension with Multi-task Learning.... Jiangnan Xia, Chen Wu, Ming Yan (Alibaba DAMO Academy)

\section{Session: Short - Health \& Sentiment}

- DIRT: Deep Learning Enhanced Item Response Theory for Cognitive Diagnosis Song Cheng, Qi Liu, Enhong Chen, Zai Huang, Zhenya Huang (University of Science and Technology of China), Yiying Chen (Ant Financial Services Group \& University of Science and Technology of China), Haiping Ma, Guoping Hu (IFLYTEK Co.,Ltd.)

- Neural Gender Prediction in Microblogging with Emotion-aware User Representation 2401 Chuhan Wu (Tsinghua University), Fangzhao Wu (Microsoft Research Asia), Tao Qi, Junxin Liu, Yongfeng Huang (Tsinghua University), Xing Xie (Microsoft Research Asia)

- Health Card Retrieval for Consumer Health Search: An Empirical Investigation of Methods..... Jimmy (University of Queensland \& University of Surabaya), Guido Zuccon (University of Queensland), Bevan Koopman (CSIRO), Gianluca Demartini (University of Queensland)

- NICE: Neural In-Hospital Cost Estimation from Medical Records 2409 Chuhan Wu (Tsinghua University), Fangzhao Wu (Microsoft Research Asia), Yongfeng Huang (Tsinghua University), Xing Xie (Microsoft Research Asia)

- Modeling Sentiment Evolution for Social Incidents. 2413 Yunjie Wang, Hui Li, Chen Lin (Xiamen University)

\section{Session: Short - Theory}

- Adaptive Feature Redundancy Minimization.

Rui Zhang (Arizona State University), Hanghang Tong (University of Illinois at Urbana-Champaign), Yifan Hu (Yahoo! Research)

- Finding a Maximum Clique in Dense Graphs via $\chi^{2}$ Statistics 2421 Sourav Dutta (Eaton Corp.), Juho Lauri (Nokia Bell Labs)

- On Heavy-user Bias in A/B Testing . 2425 Yu Wang (University of California, Berkeley), Somit Gupta, Jiannan Lu, Ali Mahmoudzadeh, Sophia Liu (Microsoft) 
- Adversarial Training of Gradient-Boosted Decision Trees

Stefano Calzavara, Claudio Lucchese (Università Ca' Foscari Venezia),

Gabriele Tolomei (Sapienza Università di Roma)

- Adversarial Structured Neural Network Pruning

Xingyu Cai (University of Connecticut), Jinfeng Yi (fD AI Research),

Fan Zhang (Zhejiang University), Sanguthevar Rajasekaran (University of Connecticut)

- Ontology-Mediated Queries over Probabilistic Data via Probabilistic Logic Programming

Timothy van Bremen, Anton Dries (KU Leuven), Jean Christoph Jung (Universität Bremen)

\section{Session: Short - Search}

- Query Embedding Learning for Context-based Social Search.

Yi-Chun Chen, Yu-Che Tsai, Cheng-Te Li (National Cheng Kung University)

- Towards More Usable Dataset Search: From Query Characterization to Snippet Generation

Jinchi Chen, Xiaxia Wang, Gong Cheng (Nanjing University),

Evgeny Kharlamov (Bosch Center for AI \& University of Oslo), Yuzhong Qu (Nanjing University)

- Session-based Search Behavior in Naturalistic Settings for Learning-related Tasks.

Souvick Ghosh (Rutgers University), Chirag Shah (University of Washington)

- Best Co-Located Community Search in Attributed Networks

Jiehuan Luo (Shenzhen Institutes of Advanced Technology, Chinese Academy of Sciences),

Xin Cao (University of New South Wales), Xike Xie (University of Science and Technology of China),

Qiang Qu (Shenzhen Institutes of Advanced Technology, Chinese Academy of Sciences)

- Caching Scores for Faster Query Processing with Dynamic Pruning in Search Engines

Erman Yafay, Ismail Sengor Altingovde (Middle East Technical University)

- Investigating the Learning Process in Job Search: A Longitudinal Study Jiaxin Mao (Tsinghua University), Damiano Spina (RMIT University),

Sargol Sadeghi (SEEK Ltd.), Falk Scholer, Mark Sanderson (RMIT University)

\section{Session: Short - System \& Database}

- Set Reconciliation with Cuckoo Filters

Lailong Luo, Deke Guo (National University of Defense Technology),

Ori Rottenstreich (Technion - Israel Institute of Technology), Richard T.B Ma (National University of Singapore),

Xueshan Luo (National University of Defense Technology)

- Shared-Nothing Distributed Enumeration of 2-Plexes.

Alessio Conte (University of Pisa),

Donatella Firmani, Maurizio Patrignani, Riccardo Torlone (Roma Tre University)

- Estimating the Number of Distinct Items in a Database by Sampling

Roel Apfelbaum (Yahoo Research)

- Cost-effective Resource Provisioning for Spark Workloads

Yuxing Chen, Jiaheng Lu (University of Helsinki), Chen Chen (Huawei Canada Research Centre),

Mohammad Hoque, Sasu Tarkoma (University of Helsinki)

- A Sampling-Based System for Approximate Big Data Analysis on Computing Clusters 2481 Salman Salloum (Shenzhen University),

Yinxu Wu (Shenzhen Institutes of Advanced Technology, Chinese Academy of Sciences \& University of Chinese Academy of Sciences), Joshua Zhexue Huang (Shenzhen University)

- TianGong-ST: A New Dataset with Large-scale Refined Real-world Web Search Sessions ... 2485 Jia Chen, Jiaxin Mao, Yiqun Liu, Min Zhang, Shaoping Ma (Tsinghua University)

\section{Session: Applied - E-commerce}

- Virtual ID Discovery from E-commerce Media at Alibaba: Exploiting Richness of User Click Behavior for Visual Search Relevance 
- Autor ${ }^{3}$ : Automated Real-time Ranking with Reinforcement Learning in E-commerce Sponsored Search Advertising .

Yusi Zhang, Zhi Yang (Peking University), Liang Wang, Li He (Alibaba Group)

- Cross-domain Attention Network with Wasserstein Regularizers for E-commerce Search.. 2509 Minghui Qiu (Alibaba Group \& Zheijiang University),

Bo Wang, Cen Chen, Xiaoyi Zeng, Jun Huang, Deng Cai, Jingren Zhou (Alibaba),

Forrest Sheng Bao (Iowa State University)

- Conceptualize and Infer User Needs in E-commerce.

Xusheng Luo, Yonghua Yang (Alibaba Group), Kenny Qili Zhu (Shanghai fiao Tong University),

Yu Gong, Keping Yang (Alibaba Group)

- Learning to Advertise for Organic Traffic Maximization in E-Commerce Product Feeds ..... 2527 Dagui Chen, Junqi Jin (Alibaba Group), Weinan Zhang (Shanghai fiao Tong University),

Fei Pan, Lvyin Niu, Chuan Yu (Alibaba Group), Jun Wang (University College London),

Han Li, Jian Xu, Kun Gai (Alibaba Group)

\section{Session: Applied - Graph Applications}

- System Deterioration Detection and Root Cause Learning on Time Series Graphs Hao Huang (GE Global Research), Shinjae Yoo (Brookhaven National Laboratory), Yunwen Xu (Amazon)

- A Dynamic Default Prediction Framework for Networked-guarantee Loans 2547 Dawei Cheng, Yiyi Zhang (Shanghai fiao Tong University), Fangzhou Yang (Seek Data Inc.),

Yi Tu (Shanghai fiao Tong University), Zhibin Niu (Tianjin University),

Liqing Zhang (Shanghai fiao Tong University)

- Feature Enhancement via User Similarities Networks for Improved Click Prediction in Yahoo Gemini Native

Morelle Arian (University of Washington),

Eliran Abutbul, Michal Aharon, Yair Koren, Oren Somekh, Rotem Stram (Yahoo Research)

- Large-Scale Visual Search with Binary Distributed Graph at Alibaba.

Kang Zhao, Pan Pan, Yun Zheng, Yanhao Zhang, Changxu Wang, Yingya Zhang,

Yinghui Xu, Rong Jin (Alibaba Group)

- Graph Representation Learning for Merchant Incentive Optimization in Mobile Payment Marketing .

Ziqi Liu, Dong Wang, Qianyu Yu, Zhiqiang Zhang, Yue Shen, Jian Ma, Wenliang Zhong,

Jinjie Gu, Jun Zhou, Shuang Yang, Yuan Qi (Ant Financial)

\section{Session: Applied - Recommendation and Advertising}

- Query-based Interactive Recommendation by Meta-Path and Adapted Attention-GRU

Yu Zhu, Yu Gong, Qingwen Liu, Yingcai Ma, Wenwu Ou, Junxiong Zhu (Alibaba Group),

Beidou Wang (State Key Laboratory of CAD\&CG, Zhejiang University), Ziyu Guan (Xidian University),

Deng Cai (State Key Laboratory of CAD\&CG, Zhejiang University)

- Learning Adaptive Display Exposure for Real-Time Advertising 2595

Weixun Wang (Tianjin University), Junqi Jin (Alibaba Group), Jianye Hao (Tianjin University),

Chunjie Chen, Chuan Yu (Alibaba Group), Weinan Zhang (Shanghai fiao Tong University),

Jun Wang (University College London), Xiaotian Hao, Yixi Wang (Tianjin University),

Han Li, Jian Xu, Kun Gai (Alibaba Group)

- What You Look Matters? Offline Evaluation of Advertising Creatives

for Cold-start Problem

Zhichen Zhao, Lei Li, Bowen Zhang, Meng Wang, Yuning Jiang, Li Xu, Fengkun Wang,

Wei-Ying Ma (Bytedance Inc)

- Multi-Interest Network with Dynamic Routing for Recommendation at Tmall

Chao Li, Zhiyuan Liu, Mengmeng Wu, Yuchi Xu, Huan Zhao, Pipei Huang (Alibaba Group),

Guoliang Kang (University of Technology Sydney), Qiwei Chen, Wei Li (Alibaba Group),

Dik Lun Lee (Hong Kong University of Science and Technology) 
- Learning to be Relevant: Evolution of a Course Recommendation System

Shivani Rao, Konstantin Salomatin, Gungor Polatkan, Mahesh Joshi,

Sneha Chaudhari, Vladislav Tcheprasov, Jeffrey Gee, Deepak Kumar (LinkedIn Corporation)

- SDM: Sequential Deep Matching Model for Online Large-scale Recommender System Fuyu Lv, Taiwei Jin (Alibaba Group), Changlong Yu (The Hong Kong University of Science and Technology), Fei Sun, Quan Lin, Keping Yang (Alibaba Group),

Wilfred Ng (The Hong Kong University of Science and Technology)

\section{Session: Applied - Urbanism and Mobility}

- Multi-Agent Reinforcement Learning for Order-dispatching via Order-Vehicle Distribution Matching... 2645 Ming Zhou, Jiarui Jin, Weinan Zhang (Shanghai fiao Tong University), Zhiwei Qin, Yan Jiao, Chenxi Wang (DiDi AI Labs), Guobin Wu (DiDi Research), Yong Yu (Shanghai fiao Tong University), Jieping Ye (DiDi AI Labs)

- MONOPOLY: Learning to Price Public Facilities for Revaluing Private Properties with Large-Scale Urban Data 2655 Miao Fan, Jizhou Huang, An Zhuo, Ying Li, Ping Li , Haifeng Wang (Baidu Inc.)

- CityTraffic: Modeling Citywide Traffic via Neural Memorization and Generalization Approach

Xiuwen Yi (FD Intelligent Cities Research, FD Intelligent Cities Business Unit, \& Tsinghua University),

Zhewen Duan (Xidian University \& FD Intelligent Cities Research, JD Intelligent Cities Business Unit),

Ting Li ( DD Intelligent Cities Research \& FD Intelligent Cities Business Unit),

Tianrui Li (Southwest Fiaotong University),

Junbo Zhang ( $(F D$ Intelligent Cities Research, $7 D$ Intelligent Cities Business Unit \& Southwest fiaotong University),

Yu Zheng (FD Intelligent Cities Research, FD Intelligent Cities Business Unit \& Southwest fiaotong University)

- Deep Dynamic Fusion Network for Traffic Accident Forecasting

Chao Huang ( $(F D$ Digits), Chuxu Zhang (University of Notre Dame), Peng Dai, Liefeng Bo (FD Digits)

- Matrix Factorization for Spatio-Temporal Neural Networks with Applications to Urban Flow Prediction

Zheyi Pan, Zhaoyuan Wang, Weifeng Wang, Yong Yu (Shanghai fiao Tong University),

Junbo Zhang ( $7 D$ Intelligent Cities Research, 7 D Intelligent Cities Business Unit \& Southwest fiaotong University),

Yu Zheng ( $F D$ Intelligent Cities Research, $7 D$ Intelligent Cities Business Unit \& Xidian University)

\section{Session: Applied - Language Models}

- Semantically Driven Auto-completion

Konstantine Arkoudas, Mohamed Yahya (Bloomberg)

- Spam Review Detection with Graph Convolutional Networks Ao Li, Zhou Qin, Runshi Liu, Yiqun Yang, Dong Li (Alibaba Group)

- Industry Specific Word Embedding and its Application in Log Classification Elham Khabiri, Wesley M. Gifford, Bhanukiran Vinzamuri, Dhaval Patel (IBM Research), Pietro Mazzoleni (IBM)

- Document-Level Multi-Aspect Sentiment Classification for Online Reviews of Medical Experts

Tian Shi (Virginia Tech), Vineeth Rakesh (Interdigital), Suhang Wang (The Pennsylvania State University),

Chandan K. Reddy (Virginia Tech)

\section{Session: Applied - Novel Applications}

- Deep Learning for Blast Furnaces: Skip-Dense Layers Deep Learning Model to Predict the Remaining Time to Close Tap-holes for Blast Furnaces 2733 Keeyoung Kim (The State University of New York (SUNY), Korea), Byeongrak Seo (IngenioAI), Sang-Hoon Rhee (Linkgenesis), Seungmoon Lee (Pohang Iron and Steel Company (POSCO)), Simon S. Woo (Sungkyunkwan University) 
- Deep Graph Similarity Learning for Brain Data Analysis

Guixiang Ma, Nesreen K. Ahmed, Theodore L. Willke, Dipanjan Sengupta (Intel Labs),

Michael W. Cole (Rutgers University), Nicholas B. Turk-Browne (Yale University),

Philip S. Yu (University of Illinois at Chicago)

- How to Find It Better? Cross-Learning for WeChat Mini Programs 2753

$\mathrm{He} \mathrm{Li}$ (Tencent Inc. \& Peking University), Zhiqiang Liu, Sheng Xu, Zhiyuan Lin (Tencent Inc.),

Xiangqun Chen (Peking University)

- Job2Vec: Job Title Benchmarking with Collective Multi-View Representation Learning ....... 2763 Denghui Zhang (Rutgers University), Junming Liu (City University of Hong Kong), Hengshu Zhu (Baidu Inc), Yanchi Liu (Rutgers University), Lichen Wang (Northeastern University), Pengyang Wang (University of Central Florida), Hui Xiong (Rutgers University \& Baidu Inc.)

- Learning to Predict Human Stress Level with Incomplete Sensor Data from Wearable Devices

Jyun-Yu Jiang, Zehan Chao, Andrea L. Bertozzi, Wei Wang, Sean D. Young,

Deanna Needell (University of California, Los Angeles)

- Fine-Grained Fuel Consumption Prediction

Chenguang Fang, Shaoxu Song, Zhiwei Chen, Acan Gui (Tsinghua University)

\section{Session: Applied - Online and User Behaviors}

- Soft Frequency Capping for Improved Ad Click Prediction in Yahoo Gemini Native Michal Aharon, Yohay Kaplan, Rina Levy, Oren Somekh (Yahoo Research), Ayelet Blanc, Neetai Eshel, Avi Shahar, Assaf Singer, Alex Zlotnik (Tech Yahoo)

- Adversarial Factorization Autoencoder for Look-alike Modeling Khoa D. Doan (Virginia Tech), Pranjul Yadav (Criteo AI Labs), Chandan K. Reddy (Virginia Tech)

- Concept Drift Adaption for Online Anomaly Detection in Structural Health Monitoring .... 2813 Hongda Tian (University of Technology Sydney), Nguyen Lu Dang Khoa (Data61 / CSIRO), Ali Anaissi (The University of Sydney), Yang Wang, Fang Chen (University of Technology Sydney)

- Multi-task based Sales Predictions for Online Promotions Shen Xin (Zhejiang University), Martin Ester (Simon Fraser University), Jiajun Bu, Chengwei Yao (Zhejiang University), Zhao Li (Alibaba Group), Xun Zhou (Zhejiang University), Yizhou Ye (Alibaba Group), Can Wang (Zhejiang University)

- Experimental Study of Multivariate Time Series Forecasting Models 2833 Jiaming Yin, Weixiong Rao (Tongji University), Mingxuan Yuan, Jia Zeng (Huawei Noah's Ark Lab), Kai Zhao (Georgia State University), Chenxi Zhang, Jiangfeng Li, Qinpei Zhao (Tongji University)

- GMTL: A GART Based Multi-task Learning Model for Multi-Social-Temporal Prediction in Online Games. 2841 Jianrong Tao, Linxia Gong, Changjie Fan (NetEase Inc.), Longbiao Chen (Xiamen University), Dezhi Ye (NetEase Inc.), Sha Zhao (Zhejiang University)

- Learning Compositional, Visual and Relational Representations for CTR Prediction in Sponsored Search 2851 Xiao Yang, Tao Deng, Weihan Tan, Xutian Tao, Junwei Zhang, Shouke Qin, Zongyao Ding (Baidu Inc.)

\section{Session: Demo - Demo Session 1}

- Inspect What Your Location History Reveals About You: Raising user awareness on privacy threats associated with disclosing his location data 2861 Antoine Boutet (Insa-Lyon / Inria), Sébastien Gambs (Uqam)

- PODIUM: Probabilistic Datalog Analysis via Contribution Maximization 2865 Tova Milo, Yuval Moskovitch, Brit Youngmann (Tel Aviv University)

- Document in Context of its Time (DICT): Providing Temporal Context to Support Analysis of Past Documents Adam Jatowt (Kyoto University \& AIRC, AIST), Ricardo Campos (Polytechnic Institute of Tomar, LIAAD-INESC TEC), Sourav S. Bhowmick (Nanyang Technological University), Antoine Doucet (University of La Rochelle) 
- ATENA: An Autonomous System for Data Exploration Based on Deep Reinforcement Learning

Ori Bar El, Tova Milo, Amit Somech (Tel Aviv University)

- ExplIQuE: Interactive Databases Exploration with SQL

Marie Le Guilly, Jean-Marc Petit, Vasile-Marian Scuturici (Univ Lyon, INSA Lyon, CNRS, LIRIS UMR 5205),

Ihab F. Ilyas (University of Waterloo)

- TraVis: An Interactive Visualization System for Mining Inbound Traveler

Activities by Leveraging Mobile Ad Request Data

Pei-Xun Wang, Hsuan Chiu, Wen-Qian Chen, Che-Chia Chang, Yu-Hsuan Huang,

Tzu-Hao Huang, Yuchun Lai, Chia-Hu Chang (Vpon Inc.)

- Understanding Data in the Blink of an Eye

Matteo Paganelli (DIEF-UNIMORE), Paolo Sottovia (DISI-UNITN),

Antonio Maccioni (Collective[i]), Matteo Interlandi (Microsoft), Francesco Guerra (DIEF - UNIMORE)

- SIMILANT: An Analytic Tool for Similarity Modeling

David Bernhauer (Czech Technical University in Prague \& Charles University),

Tomáš Skopal, Irena Holubová, Ladislav Peška, Martin Svoboda (Charles University)

- MithraLabel: Flexible Dataset Nutritional Labels for Responsible Data Science

Chenkai Sun (University of Michigan), Abolfazl Asudeh (University of Illinois at Chicago),

H. V. Jagadish (University of Michigan), Bill Howe (University of Washington),

Julia Stoyanovich (New York University)

- PRIVATA: Differentially Private Data Market Framework using Negotiation-based Pricing Mechanism

Kangsoo Jung, Junkyu Lee, Kunyoung Park, Seog Park (Sogang University)

- MiCRon: Making Sense of News via Relationship Subgraphs

Zixian Huang, Shuxin Li, Gong Cheng (Nanjing University),

Evgeny Kharlamov (Bosch Center for AI), Yuzhong Qu (Nanjing University)

- LuPe: A System for Personalized and Transparent Data-driven Decisions 2905

Sarah Oppold, Melanie Herschel (University of Stuttgart)

- Insta-Search: Towards Effective Exploration of Knowledge Graphs

Madhulika Mohanty, Maya Ramanath (Indian Institute of Technology, Delhi)

\section{Session: Demo - Demo Session 2}

- SkyRec: Finding Pareto Optimal Groups

Jinfei Liu (Emory University \& Georgia Institute of Technology), Li Xiong (Emory University),

Jian Pei (Simon Fraser University), Jun Luo (Machine Intelligence Center Lenovo Group Limited),

Haoyu Zhang (Indiana University Bloomington), Si Zhang (fianghan University)

- CurrentClean: Interactive Change Exploration and Cleaning of Stale Data

Zheng Zheng, Tri Minh Quach, Ziyi Jin, Fei Chiang (McMaster University),

Mostafa Milani (The University of British Columbia)

- PatMat: A Distributed Pattern Matching Engine with Cypher Kongzhang Hao, Zhengyi Yang, Longbin Lai (University of New South Wales), Zhengmin Lai, Xin Jin (ECNU), Xuemin Lin (University of New South Wales)

- On a Chatbot Conducting Virtual Dialogues

Boris Galitsky (Oracle Inc.), Dmitry Ilvovsky (National Research University Higher School of Economics)

- Rehab-Path: Recommending Alcohol and Drug-free Routes

Yihong Zhang (Osaka University), Panote Siriaraya, Yukiko Kawai (Kyoto Sangyo University),

Adam Jatowt (Kyoto University)

- kBrowse: kNN Graph Browser.

Ramon Bespinyowong, Anthony K. H. Tung (National University of Singapore) 
- BIP! Finder: Facilitating Scientific Literature Search by Exploiting Impact-Based Ranking.

Thanasis Vergoulis (IMSI, Athena RC),

Serafeim Chatzopoulos (University of the Peloponnese \& IMSI, Athena RC),

Ilias Kanellos (IMSI, Athena RC),

Panagiotis Deligiannis, Christos Tryfonopoulos (University of the Peloponnese),

Theodore Dalamagas (IMSI, Athena RC)

- BeLink: Querying Networks of Facts, Statements and Beliefs.

Tien-Duc Cao (Inria and LIX (UMR 7161, CNRS and Ecole polytechnique)),

Ludivine Duroyon, François Goasdoué (Univ Rennes, CNRS, Inria, IRISA),

Ioana Manolescu (Inria and LIX (UMR 7161, CNRS and Ecole polytechnique)),

Xavier Tannier (Sorbonne Université and Inria)

- TuneR: Fine Tuning of Rule-based Entity Matchers

Matteo Paganelli (DIEF-UNIMORE), Paolo Sottovia (DISI-UNITN), Francesco Guerra (DIEF-UNIMORE),

Yannis Velegrakis (Utrecht University)

- I-REX: A Lucene Plugin for EXplainable IR

Dwaipayan Roy (GESIS - Leibniz Institute for the Social Sciences),

Sourav Saha, Mandar Mitra (Indian Statistical Institute),

Bihan Sen (Microsoft, India), Debasis Ganguly (IBM Research Lab)

- Model Asset eXchange: Path to Ubiquitous Deep Learning Deployment

Alex Bozarth, Brendan Dwyer, Fei Hu, Daniel Jalova, Karthik Muthuraman, Nick Pentreath, Simon Plovyt,

Gabriela de Queiroz, Saishruthi Swaminathan, Patrick Titzler, Xin Wu, Hong Xu, Frederick R. Reiss,

Vijay Bommireddipalli (IBM)

- ReducE-Comm: Effective Inventory Reduction System for E-Commerce.

Shay Gershtein, Tova Milo (Tel Aviv University), Slava Novgorodov (eBay Research)

- dEFEND: A System for Explainable Fake News Detection

Limeng Cui (Penn State University), Kai Shu (Arizona State University),

Suhang Wang, Dongwon Lee (Penn State University), Huan Liu (Arizona State University)

\section{Tutorials}

- Enterprise Knowledge Graph From Specific Business Task to Enterprise Knowledge Management...

Rong Duan (Huawei Technology), Yanghua Xiao (Fudan University)

- Taming Social Bots: Detection, Exploration and Measurement

Abdullah Mueen (University of New Mexico), Nikan Chavoshi (Oracle Corporation),

Amanda Minnich (Lawrence Livermore National Laboratories)

- Learning-Based Methods with Human-in-the-Loop for Entity Resolution

Sairam Gurajada, Lucian Popa, Kun Qian, Prithviraj Sen (IBM Research - Almaden)

- Learning and Reasoning on Graph for Recommendation

Xiang Wang (National University of Singapore),

Xiangnan He (University of Science and Technology of China),

Tat-Seng Chua (National University of Singapore)

- Recent Developments of Deep Heterogeneous Information Network Analysis

2973

Chuan Shi (Beijing University of Posts and Telecommunications),

Philip S. Yu (University of Illinois at Chicago)

- Synergy of Database Techniques and Machine Learning Models for String Similarity Search and Join...

Jiaheng Lu (University of Helsinki), Chunbin Lin (Amazon AWS),

Jin Wang (University of California, Los Angeles), Chen Li (University of California Irvine)

- Realtime Object Detection via Deep Learning-based Pipelines 2977 James G. Shanahan (Bryant University \& University of California, Berkeley), Liang Dai (Facebook \& University of California, Santa Cruz) 
- Recommendation for Multi-stakeholders and through Neural Review Mining

Muthusamy Chelliah (Flipkart), Yong Zheng (Illinois Institute of Technology),

Sudeshna Sarkar (Indian Institute of Technology)

- Machine Learning on Graphs with Kernels

Michalis Vazirgiannis (École Polytechnique \& AUEB), Giannis Nikolentzos (École Polytechnique),

Giannis Siglidis (Sorbonne Université)

\section{Workshop Summaries}

- DTMBIO 2019: The Thirteenth International Workshop on Data and Text Mining in Biomedical Informatics.

Hyojung Paik (KISTI), Ruibin Xi (Peking University),

Doheon Lee (Korea Advanced Institute of Science \& Technology)

- Knowledge-Driven Analytics and Systems Impacting Human Quality of Life

Arijit Ukil (Tata Consultancy Services), Leandro Marin (University of Murcia),

Antonio Jara (University of Applied Sciences Western Switzerland (HES-SO)),

John Farserotu (Centre Suisse d'Electronique et de Microtechnique (CSEM))

- HENA 2019: The 3rd Workshop of Heterogeneous Information Network

Analysis and Applications

Chuan Shi (Beijing University of Posts and Telecommunications),

Yanfang Ye (Case Western Reserve University), Jiawei Zhang (Florida State University)

- EYRE 2019: 2nd International Workshop on EntitY REtrieval.

Gong Cheng (Nanjing University), Kalpa Gunaratna (Samsung Research America),

Jun Wang (University College London)

- CIKM 2019 Workshop on Artificial Intelligence in Transportation (AI in transportation).... 2995 Weinan Zhang, Haiming Jin (Shanghai fiao Tong University),

Lingyu Zhang, Hongtu Zhu (AI Labs, DiDi),

Jessie Zhenhui Li (The Pennsylvania State University), Jieping Ye (AI Labs, DiDi)

- GRLA 2019: The first International Workshop on Graph Representation Learning and its Applications

Huawei Shen (CAS Key Laboratory of Network Data Science and Technology, Institute of Computing Technology,

Chinese Academy of Sciences),

Jian Tang (HEC Montreal Montreal Institute for Learning Alogorithms),

Peng Bao (School of Software Engineering, Beijing fiaotong University)

- International Workshop on Model Selection and Parameter Tuning in Recommender Systems 2999

Fikret Sivrikaya (GT-ARC gemeinnützige GmbH), Sahin Albayrak (Technische Universität Berlin),

Defu Lian (University of Science and Technology of China)

- 2nd Workshop on Knowledge-aware and Conversational Recommender Systems - KaRS .. 3001 Vito Walter Anelli, Tommaso Di Noia (Polytechnic University of Bari)

- BigScholar 2019: The 6th Workshop on Big Scholarly Data 3003

Feng Xia (Dalian University of Technology), Huan Liu (Arizona State University), Irwin King (The Chinese University of Hong Kong), Kuansan Wang (Microsoft Research) 


\section{CIKM 2019 Conference Organization}

Honorable Chairs: Hong Mei (Beijing Institute of Technology, China)

Ramamohanarao Kotagiri (The University of Melbourne, Australia)

General Chairs: Wenwu Zhu (Tsinghua University, China)

Dacheng Tao (The University of Sydney, Australia)

Xueqi Cheng (Institute of Computing Technology, CAS, China)

Program Chairs: Peng Cui (Tsinghua University, China)

Elke Rundensteiner (Worcester Polytechnic Institute, USA)

David Carmel (Amazon Research, USA)

Qi He (LinkedIn, USA)

Jeffrey $\mathrm{Xu}$ Yu (Chinese University of Hong Kong, China)

Industry Track Chairs: Paul Ogilvie (Databricks, USA)

Ji-Rong Wen (Renmin University of China, China)

Tutorial Chairs: Leman Akoglu (Carnegie Mellon University, USA)

Enhong Chen (University of Science \& Technology of China, China)

Workshop Chairs: Minling Zhang (Southeast University, China)

Jingrui He (UIUC, USA)

Demo Chairs: Danai Koutra (University of Michigan, USA)

Fei Wang (Cornell University, USA)

Student Travel Grant Chair: Yan Liu (University of Southern California, USA)

Publicity Chairs: William Wang (UCSB, USA)

Zhiyuan Liu (Tsinghua University, China)

Publication Chairs: Meng Jiang (University of Notre Dame, USA)

Mu Qiao (IBM Research - Almaden, USA)

Local Chairs: Huawei Shen (Chinese Academy of Sciences, China)

Xin Zhao (Renmin University of China, China)

Jiafeng Guo (Chinese Academy of Sciences, China)

Wie Cui (Squirrel AI Learning, China)

Sponsor Chairs: Shiqiang Yang (Tsinghua University, China)

Weinan Zhang (Shanghai Jiao Tong University, China)

Web Chair: Jingyuan Wang (Beihang University, China)

Registration Chairs: Chuan Shi (Beijing University of Posts and Telecommunications, China) 


\section{CIKM'19 Senior Program Committee - Long Track}

Eugene Agichtein (Emory University, USA)

Deng Cai (Zhejiang University, China)

Selcuk Candan Reviewer I (Arizona State University, USA)

Ben Carterette (University of Delaware, USA)

Carlos Castillo (Universitat Pompeu Fabra, Spain)

Yi Chang (School of Artificial Intelligence, Jilin University, China, USA)

Hong Cheng (The Chinese University of Hong Kong, Hong Kong)

Charles Clarke (Facebook, Canada)

Shane Culpepper (RMIT University, Australia)

Brian Davison (Lehigh University, USA)

Laura Dietz (University of New Hampshire, USA)

Wei Ding (University of Massachusetts Boston, USA)

Xin Dong (Amazon, USA)

Bo Du (Wuhan University, China)

Hong Gao (Harbin Institute of Technology, China)

Jing Gao (University at Buffalo, USA)

Souvik Ghosh (LinkedIn, USA)

Aristides Gionis (Aalto University, Finland)

Jiafeng Guo (Institute of Computing Technology, China)

Ido Guy (eBay Research, Israel)

Jiawei Han (University of Illinois at UrbanaChampaign, USA)

Wook-Shin Han (POSTECH, South Korea)

Claudia Hauff (Delft University of Technology, Netherlands)

Bingsheng He (National University of Singapore, Singapore)

Xiangnan He (University of Science \& Technology of China, China)

Xia Ben Hu (Texas A\&M University, USA)

Zi Huang (The University of Queensland, Australia)

Shuiwang Ji (Texas A\&M University, USA)

Meng Jiang (University of Notre Dame, USA)

Jaap Kamps (University of Amsterdam, Netherlands)

Krishnaram Kenthapadi (LinkedIn Corporation, USA)

Udo Kruschwitz (University of Regensburg, Germany)

Oren Kurland (Technion, Israel Institute of Technology, Israel)

Chenliang Li (Wuhan University, China)
Guoliang Li (Tsinghua University, China)

Hang Li (Bytedance AI Lab, China)

Jiuyong Li (University of South Australia, Australia)

Zhenhui Jessie Li (The Pennsylvania State University, USA)

Ee-Peng Lim (Singapore Management University, Singapore)

Jimmy Lin (University of Waterloo, Canada)

Huan Liu (Arizona State University, USA)

Tongliang Liu (The University of Sydney, Australia)

Wei Liu (Tencent, USA)

Yiqun Liu (Tsinghua University, China)

Chang-Tien Lu (Virginia Tech, USA)

Alistair Moffat (The University of Melbourne,

Australia)

Wolfgang Nejdl (L3S and University of Hannover,

Germany)

Charles Nicholas (UMBC, USA)

Vagelis Papalexakis (University of California

Riverside, USA)

Jian Pei (Simon Fraser University, Canada)

Bryan Perozzi (Google, USA)

Buyue Qian (Xi'an Jiaotong University, China)

Weining Qian (East China Normal University, China)

Naren Ramakrishnan (Virginia Tech, USA)

Chandan K. Reddy (Virginia Tech, USA)

Mark Sanderson (RMIT University, Australia)

Rodrygo Santos (Universidade Federal de Minas

Gerais, Brazil)

Pavel Serdyukov (Yandex, Russia)

Huawei Shen (Chinese Academy of Sciences, China)

Guojie Song (Peking University, Beijing, China,

China)

Yangqiu Song (The Hong Kong University of Science

\& Technology, Hong Kong)

Aixin Sun (Nanyang Technological University, Singapore)

Kian-Lee Tan (National University of Singapore,

Singapore)

Jie Tang (Tsinghua University, China)

Hanghang Tong (University of Illinois at Urbana-

Champaign, USA)

Yongxin Tong (Beihang University, China)

Andrew Trotman (University of Otago, New Zealand) 
Vincent Tseng (National Chiao Tung University, Taiwan)

Fei Wang (Cornell University, USA)

Hao Wang (Norwegian University of Science \& Technology, Norway)

Ke Wang (Simon Fraser University, Canada)

Wei Wang (University of California, Los Angeles, USA)

Ji-Rong Wen (Renmin University of China, China)

Yonggang Wen (Nanyang Technological University, Singapore)

Ryen White (Microsoft, USA)

Xiaokui Xiao (National University of Singapore, Singapore)

Yuan Xie (Institute of Automation, Chinese Academy of Sciences, China)

Hui Xiong (Rutgers University, USA)
Jianliang Xu (Hong Kong Baptist University, Kowloon Tong, Hong Kong)

Junchi Yan (Shanghai Jiao Tong University, China)

Jaewon Yang (Stanford University, USA)

Xiaochun Yang (Northeastern University, China)

Jieping Ye (DiDi Chuxing, China)

Philip Yu (University of Illinois at Chicago, USA)

Liang Zhang (LinkedIn, USA)

Wenjie Zhang (unsw, Australia)

Ying Zhang (University of Technology, Sydney, Australia)

Zhi-Hua Zhou (Nanjing University, China)

Feida Zhu (Singapore Management University, Singapore)

Guido Zuccon (The University of Queensland, Australia) 


\section{CIKM'19 Senior Program Committee - Short Track}

Eugene Agichtein (Emory University, USA)

Deng Cai (Zhejiang University, China)

Kasim Candan (Arizona State University, USA)

Ben Carterette (University of Delaware, USA)

Carlos Castillo (Universitat Pompeu Fabra, Spain)

Yi Chang (School of Artificial Intelligence, Jilin

University, China, USA)

Hong Cheng (The Chinese University of Hong Kong, Hong Kong)

Charles Clarke (Facebook, Canada)

Shane Culpepper (RMIT University, Australia)

Brian Davison (Lehigh University, USA)

Laura Dietz (University of New Hampshire, USA)

Wei Ding (University of Massachusetts Boston, USA)

Xin Dong (Amazon, USA)

Bo Du (Wuhan University, China)

Hong Gao (Harbin Institute of Technology, China)

Jing Gao (University at Buffalo, USA)

Souvik Ghosh (LinkedIn, USA)

Aristides Gionis (Aalto University, Finland)

Jiafeng Guo (Institute of Computing Technology,

China)

Ido Guy (eBay Research, Israel)

Jiawei Han (University of Illinois at Urbana-

Champaign, USA)

Wook-Shin Han (POSTECH, South Korea)

Claudia Hauff (Delft University of Technology,

Netherlands)

Bingsheng He (National University of Singapore, Singapore)

Xiangnan He (University of Science \& Technology of China, China)

Zi Huang (The University of Queensland, Australia)

Shuiwang Ji (Texas A\&M University, USA)

Meng Jiang (University of Notre Dame, USA)

Jaap Kamps (University of Amsterdam, Netherlands)

Krishnaram Kenthapadi (LinkedIn Corporation, USA)

Udo Kruschwitz (University of Regensburg,

Germany)

Oren Kurland (Technion, Israel Institute of

Technology, Israel)

Chenliang Li (Wuhan University, China)

Guoliang Li (Tsinghua University, China)

Hang Li (Bytedance AI Lab, China)
Jiuyong Li (University of South Australia, Australia)

Zhenhui Li (The Pennsylvania State University, USA)

Ee-Peng Lim (Singapore Management University, Singapore)

Jimmy Lin (University of Waterloo, Canada)

Huan Liu (Arizona State University, USA)

Tongliang Liu (The University of Sydney, Australia)

Wei Liu (Tencent, USA)

Yiqun Liu (Tsinghua University, China)

Chang-Tien Lu (Virginia Tech, USA)

Alistair Moffat (The University of Melbourne, Australia)

Wolfgang Nejdl (L3S and University of Hannover, Germany)

Charles Nicholas (UMBC, USA)

Vagelis Papalexakis (University of California

Riverside, USA)

Jian Pei (Simon Fraser University, Canada)

Bryan Perozzi (Google, USA)

Buyue Qian (Xi'an Jiaotong University, China)

Weining Qian (East China Normal University, China)

Naren Ramakrishnan (Virginia Tech, USA)

Chandan Reddy (Virginia Tech, USA)

Mark Sanderson (RMIT University, Australia)

Rodrygo Santos (Universidade Federal de Minas

Gerais, Brazil)

Pavel Serdyukov (Yandex, Russia)

Huawei Shen (Chinese Academy of Sciences, China)

Guojie Song (Room 2307 in Science Building Two,

Peking University, Beijing, China, China)

Yangqiu Song (The Hong Kong University of Science \& Technology, Hong Kong)

Aixin Sun (Nanyang Technological University, Singapore)

Kian-Lee Tan (National University of Singapore, Singapore)

Jie Tang (Tsinghua University, China)

Hanghang Tong (University of Illinois at Urbana-

Champaign, USA)

Yongxin Tong (Beihang University, China)

Andrew Trotman (University of Otago, New Zealand)

Vincent Tseng (National Chiao Tung University,

Taiwan)

Fei Wang (Cornell University, USA) 
Hao Wang (Norwegian University of Science \& Technology, Norway)

Ke Wang (Simon Fraser University, Canada)

Wei Wang (University of California, Los Angeles, USA)

Ji-Rong Wen (Renmin University of China, China)

Yonggang Wen (Nanyang Technological University, Singapore)

Ryen White (Microsoft, USA)

Xiaokui Xiao (National University of Singapore, Singapore)

Yuan Xie (Institute of Automation, Chinese Academy of Sciences, China)

Hui Xiong (Rutgers University, USA)

Jianliang Xu (Hong Kong Baptist University, Kowloon Tong, Hong Kong)
Junchi Yan (Shanghai Jiao Tong University, China) Jaewon Yang (Stanford University, USA)

Xiaochun Yang (Northeastern University, China)

Jieping Ye (DiDi Chuxing, China)

Philip Yu (University of Illinois at Chicago, USA)

Liang Zhang (LinkedIn, USA)

Wenjie Zhang (unsw, Australia)

Ying Zhang (University of Technology, Sydney, Australia)

Zhi-Hua Zhou (Nanjing University, China)

Feida Zhu (Singapore Management University, Singapore)

Guido Zuccon (The University of Queensland, Australia) 


\section{CIKM'19 Senior Program Committee - Applied Track}

Kasim Candan (Arizona State University, USA)

Ben Carterette (University of Delaware, USA)

Yi Chang (School of Artificial Intelligence, Jilin University, China)

Hong Cheng (The Chinese University of Hong Kong, Hong Kong)

Shane Culpepper (RMIT University, Australia)

Wei Ding (University of Massachusetts Boston, USA)

Bo Du (Wuhan University, China)

Hong Gao (Harbin Institute of Technology, China)

Jing Gao (University at Buffalo, USA)

Bingsheng He (National University of Singapore, Singapore)

Xiangnan He (University of Science \& Technology of China, China)

Zi Huang (The University of Queensland, Australia)

Yantao Jia (Institute of Computing Technology, CAS \& Huawei Technologies, China)

Meng Jiang (University of Notre Dame, USA)

Jaap Kamps (University of Amsterdam, Netherlands)

Udo Kruschwitz (University of Regensburg, Germany)

Chenliang Li (NTU, Singapore)

Lei Li (ByteDance AI Lab, USA)

Zhenhui Jessie Li (The Pennsylvania State University, USA)

Huan Liu (Arizona State University, USA)

Tongliang Liu (The University of Sydney, Australia)

Yiqun Liu (Tsinghua University, China)

Wolfgang Nejdl (L3S and University of Hannover, Germany)

Charles Nicholas (UMBC, USA)
Buyue Qian (Xi'an Jiaotong University, China)

Chandan K. Reddy (Virginia Tech, USA)

Dongjin Song (NEC Labs America, USA)

Kian-Lee Tan (National University of Singapore, Singapore)

Jianrong Tao (NetEase Fuxi AI Lab, China)

Hanghang Tong (University of Illinois at Urbana-

Champaign, USA)

Yongxin Tong (Beihang University, China)

Andrew Trotman (University of Otago, New Zealand)

Vincent Tseng (National Chiao Tung University, Taiwan)

Hao Wang (Norwegian University of Science \&

Technology, Norway)

Ryen White (Microsoft, USA)

Xiaokui Xiao (National University of Singapore, Singapore)

Hui Xiong (Rutgers University, USA)

Jianliang Xu (Hong Kong Baptist University,

Kowloon Tong, Hong Kong)

Junchi Yan (Shanghai Jiao Tong University, China)

Hongxia Yang (Alibaba, China)

Xiaochun Yang (Northeastern University, China)

Philip Yu (University of Illinois at Chicago, USA)

Wenjie Zhang (University of New South Wales, Australia)

Ying Zhang (University of Technology, Sydney, Australia)

Feida Zhu (Singapore Management University, Singapore)

Guido Zuccon (The University of Queensland, Australia) 


\section{CIKM'19 Program Committee Members - Long Track}

Ziawasch Abedjan (TU Berlin, Germany)

Karl Aberer (Ecole Polytechnique Fédérale de

Lausanne, Switzerland)

Ayan Acharya (CognitiveScale Inc., USA)

Ashvin Agrawal (Microsoft, USA)

Ismail Sengor Altingovde (Middle East Technical

University, Turkey)

Giambattista Amati (Fondazione Ugo Bordoni, Italy)

Enrique Amigó (UNED, Spain)

Avi Arampatzis (Democritus University of Thrace,

Greece)

Walid Aref (Purdue University, USA)

Renato Assuncao (UFMG, Brazil)

Hosein Azarbonyad (University of Amsterdam,

Netherlands)

Xiao Bai (Yahoo Research, USA)

Leandro Balby Marinho (Federal University of

Campina Grande (Federal University of Campina

Grande (UFCG), Brazil)

Peng Bao (Beijing Jiaotong University, China)

Ziawasch Abedjan (TU Berlin, Germany)

Karl Aberer (Ecole Polytechnique Fédérale de

Lausanne, Switzerland)

Ayan Acharya (CognitiveScale Inc., USA)

Ashvin Agrawal (Microsoft, USA)

Ismail Sengor Altingovde (Middle East Technical

University, Turkey)

Giambattista Amati (Fondazione Ugo Bordoni, Italy)

Enrique Amigó (UNED, Spain)

Avi Arampatzis (Democritus University of Thrace,

Greece)

Walid Aref (Purdue University, USA)

Renato Assuncao (UFMG, Brazil)

Hosein Azarbonyad (University of Amsterdam, Netherlands)

Xiao Bai (Yahoo Research, USA)

Leandro Balby Marinho (Federal University of

Campina Grande (UFCG), Brazil)

Peng Bao (Beijing Jiaotong University, China)

Senjuti Basu Roy (New Jersey Institute of

Technology, USA)

Roberto Bayardo (Google, USA)

Ryan Benton (University of South Alabama, USA)

Michele Berlingerio (IBM, Ireland)
Elisa Bertino (Purdue University, USA)

Sumit Bhatia (IBM, India)

Siddhartha Bhattacharyya (University of Illinois,

Chicago, USA)

Jiang Bian (Microsoft, China)

Asia J. Biega (Microsoft, Canada)

Bodo Billerbeck (Bing, Australia)

Francesco Bonchi (Fondazione ISI, Italy)

Ilaria Bordino (UniCredit R\&D, Italy)

Renata Borovica-Gajic (The University of Melbourne,

Australia)

Marco Brambilla (Politecnico di Milano, Italy)

Pavel Braslavski (Ural Federal University, Russia)

Ivan Brugere (Salesforce Research, USA)

François Bry (Ludwig Maximilian University of

Munich, Germany)

Yingyi Bu (Google, USA)

Klemens Böhm (Karlsruhe Institute of Technology,

Germany)

Fidel Cacheda (Universidade da Coruña, Spain)

Jose Cadena (Lawrence Livermore National

Laboratory, USA)

Xiangrui Cai (Nankai University, China)

Zhipeng Cai (Georgia State University, USA)

Fazli Can (Bilkent University, Turkey)

Huiping Cao (New Mexico State University, USA)

Jian Cao (Shanghai Jiao Tong University, China)

Lei Cao (Massachusetts Institute of Technology, USA)

Longbing Cao (University of Technology Sydney,

Australia)

Xin Cao (The University of New South Wales,

Australia)

Yang Cao (The University of Edinburgh, UK)

Bogdan Cautis (ENST Paris - UMR CNRS 5141,

France)

James Caverlee (Texas A\&M University, USA)

Michelangelo Ceci (Universita degli Studi di Bari, Italy)

Tanmoy Chakraborty (IIIT-Delhi, India, India)

Sharma Chakravarthy (The University of Texas at

Arlington, USA)

Jeffrey Chan (RMIT University, Australia)

Keith C.C. Chan (The Hong Kong Polytechnic

University, Hong Kong) 
Philip Chan (Florida Institute of Technology, USA)

Praveen Chandar (Spotify Research, USA)

Kevin Chang (University of Illinois at Urbana-

Champaign, USA)

Abon Chaudhuri (WalmartLabs, USA)

Muhammad Aamir Cheema (Monash University, Australia)

Chen Chen (Arizona State University, USA)

Feng Chen (University at Albany - SUNY, USA)

Hsin-Hsi Chen (National Taiwan University, Taiwan)

Huanhuan Chen (University of Science \& Technology of China, China)

Huiyuan Chen (Case Western Reserve University, USA)

Jinjun Chen (University of Technology, Sydney, Australia)

Ling Chen (University of Technology, Sydney, Australia)

Lisi Chen (Hong Kong Baptist University, Hong Kong)

Ping Chen (UMB, USA)

Qian Chen (Google, China)

Ruey-Cheng Chen (SEEK Ltd, Australia)

Rui Chen (Samsung Electronics, USA)

Shu-Ching Chen (Florida International University, USA)

Xilun Chen (Arizona State University, USA)

$\mathrm{Xu}$ Chen (Tsinghua University, China)

Yang Chen (LinkedIn, USA)

Zhengzhang Chen (NEC Laboratories America, USA)

Zhenzhong Chen (Wuhan University, China)

Zhiyuan Chen (University of Maryland Baltimore County, USA)

Zhumin Chen (shandong university, China)

Dawei Cheng (Shanghai Jiao Tong University, China)

Yurong Cheng (Beijing Institute of Technology, China)

Fei Chiang (McMaster University, Canada)

Adrian-Gabriel Chifu (Aix Marseille Univ, Université de Toulon, CNRS, LIS, France)

Silvia Chiusano (Politecnico di Torino, Italy)

Vassilis Christophides (INRIA, France)

Panos Chrysanthis (University of Pittsburgh, USA)

Lingyang Chu (Simon Fraser University, Canada)

Korris Chung (The Hong Kong Polytechnic University, China)
Arman Cohan (Allen Institute for Artificial Intelligence (AI2), USA)

Nachshon Cohen (Ecole Polytechnique Fédérale de Lausanne, Switzerland)

Michael Cole (LexisNexis, USA)

Gao Cong (Nanyang Technological University, Singapore)

Michele Coscia (IT University of Copenhagen, Denmark)

Matt Crane (Facebook, USA)

Bruno Cremilleux (Universite de Caen Normandie, France)

Paolo Cremonesi (Politecnico di Milano, Italy)

Philippe Cudre-Mauroux (University of Fribourg, Switzerland)

Tonya Custis (Thomson Reuters, USA)

Alfredo Cuzzocrea (ICAR-CNR and University of Calabria, Italy)

Arthur Câmara (Delft University of Technology, Netherlands)

Hong-Ning Dai (Macau Univ. of Sci. and Tech., Macao)

Hongliang Dai (The Hong Kong University of Science \& Technology, Hong Kong)

Mahashweta Das (Visa Research, USA)

Anwitaman Datta (Nanyang Technological University, Singapore)

Edleno Silva De Moura (Universidade Federal do Amazonas, Brazil)

Maarten de Rijke (University of Amsterdam, Netherlands)

Cheng Deng (Xidian University, China)

Shuiguang Deng (Zhejiang University, China)

Bolin Ding (Alibaba Group, USA)

Jingtao Ding (Tsinghua University, Beijing,

P.R.China, China)

Yao-Xiang Ding (Nanjing University, China)

Ying Ding (Indiana University Bloomington, USA)

Nemanja Djuric (Uber ATG, USA)

Josep Domingo-Ferrer (Universitat Rovira i Virgili, Spain)

Yuxiao Dong (Microsoft, USA)

Dejing Dou (University of Oregon, USA)

Zhicheng Dou (Renmin University of China, China)

Peng Du (LinkedIn Corporation, USA)

Tamer Elsayed (Qatar University, Qatar)

Mohamed Eltabakh (WPI, USA) 
Vlad Estivill-Castro (Griffith University, Australia)

Xuhui Fan (UTS, Australia)

Yi Fang (Santa Clara University, USA)

Yixiang Fang (The University of New South Wales, Australia)

Yuan Fang (Singapore Management University, Singapore)

Fuli Feng (National University of Singapore, Singapore)

Qingxiang Feng (Tulane University, USA)

Yunlong Feng (SUNY Albany, USA)

Hakan Ferhatosmanoglu (The University of Warwick, UK)

Elena Ferrari (University of Insubria, Italy)

Donatella Firmani (Roma Tre University, Italy)

Peter M. Fischer (Universität Augsburg, Institut für Informatik, Germany)

George H. L. Fletcher (Eindhoven University of Technology, Netherlands)

Edward Fox (Virginia Tech, USA)

Marios Fragkoulis (AUEB, Greece)

Yanjie Fu (University of Central Florida, USA)

Luke Gallagher (RMIT University, Australia)

Junhao Gan (The University of Melbourne, Australia)

Byron Gao (Texas State University, USA)

Chen Gao (Tsinghua University, China)

Jun Gao (none, China)

Min Gao (Chongqing University, China)

Yue Gao (Tsinghua University, China)

Yash Garg (Arizona State University, USA)

Wolfgang Gatterbauer (Northeastern University, USA)

Tingjian Ge (University of Massachusetts, Lowell, USA)

Dimitrios Georgakopoulos (Swinburne University of Technology, Australia)

Sahin Geyik (LinkedIn, USA)

Aris Gkoulalas-Divanis (IBM Watson Health,

Cambridge, MA, USA, USA)

Boris Glavic (Illinois Institute of Technology, USA)

Marcos Goncalves (Federal University of Minas

Gerais, Brazil)

Dihong Gong (UF, USA)

Mingming Gong (University of Pittsburgh, USA)

Julio Gonzalo (UNED, Spain)

Sergio Greco (University of Calabria, Italy)

Ziyu Guan (Northwest University of China, China)
Jie Gui (hfcas, China)

Francesco Gullo (UniCredit, Italy)

Qi Guo (LinkedIn, USA)

Parth Gupta (Amazon, Spain)

Jialong Han (Nanyang Technological University,

Singapore)

Qilong Han (College of Computer Science \&

Technology,Harbin Engineering University,

China)

Ben He (University of Chinese Academy of Sciences, China)

Dongxiao He (Tianjin University, China)

Jingrui He (University of Illinois at Urbana-

Champaign, USA)

Jiong He (Alibaba Group, Singapore)

Lifang He (University of Pennsylvania, USA)

Xiaofeng He (East China Normal University, China)

Yeye He (Microsoft, USA)

Zhenying He (Fudan University, China)

Sven Helmer (University of Zurich, Switzerland)

Heli Helskyaho (Miracle Finland Oy, Finland)

Shen-Shyang Ho (Rowan University, USA)

Tuan-Anh Hoang (L3S Research Center, Leibniz

University of Hanover, Germany)

Liangjie Hong (Etsy Inc., USA)

Frank Hopfgartner (The University of Sheffield, UK)

Bill Howe (University of Washington, USA)

$\mathrm{Han} \mathrm{Hu}$ (Beijing Institute of Technology, China, China)

Juhua $\mathrm{Hu}$ (University of Washington | Tacoma, USA)

Ziniu Hu (University of California, Los Angeles, USA)

Kaixun Hua (University of Massachusetts Boston, USA)

Wen Hua (The University of Queensland, Australia)

Chao Huang (University of Notre Dame, USA)

Jin Huang (Tsinghua University, China)

Joshua Huang (Shenzhen University, China)

Wei Huang (State Key Lab of Software

Engineering,Wuhan University, China)

Xiao Huang (Texas A\&M University, USA)

Xin Huang (Hong Kong Baptist University, Hong

Kong)

Xuanjing Huang (Fudan University, China)

Chih-Chieh Hung (Tamkang University, Taiwan)

Tsuyoshi Ide (IBM, USA)

Sam Idicula (Oracle, USA)

Amir Ingber (Amazon Research, Israel) 
Stratis Ioannidis (Northeastern University, USA)

Radu Tudor Ionescu (University of Bucharest, Faculty of Mathematics and Computer Science, Romania)

M. Raihanul Islam (Virginia Polytechnic Institute, USA)

Feng Ji (DAMO Academy, Alibaba Group, China)

Ling Jian (China University of Petroleum, China)

Daxin Jiang (Microsoft, China)

Di Jiang (WeBank, China)

Wenhao Jiang (Tencent, China)

Bo Jin (Dalian University of Technology, China)

Di Jin (Tianjin University, China)

Ruoming Jin (Kent State University, USA)

Alekh Jindal (Microsoft, USA)

Gareth Jones (Dublin City University, Ireland)

Roy Ka-Wei Lee (Singapore Management University, Singapore)

Panos Kalnis (King Abdullah University of Science \& Technology, Saudi Arabia)

Konstantinos Kalpakis (UMBC, USA)

Bhargav Kanagal (Google, USA)

U Kang (Seoul National University, South Korea)

Zhao Kang (southern illinois university carbondale, USA)

Verena Kantere (University of Ottawa, Canada)

Ben Kao (The University of Hong Kong, Hong Kong)

Zoi Kaoudi (Qatar Computing Research Institute, Qatar)

Kamalakar Karlapalem (CDE, IIIT Hyderabad, India, India)

Panagiotis Karras (Aarhus University, Denmark)

Harshad Kasture (Oracle Labs, USA)

Makoto P. Kato (University of Tsukuba, Japan)

Tom Kenter (University of Amsterdam, Netherlands)

Eamonn Keogh (UC Riverside, USA)

Maurice van Keulen (University of Twente, Netherlands)

Arijit Khan (Nanyang Technological University, Singapore)

Myunghwan Kim (LinkedIn Corporation, USA)

Sang-Wook Kim (Hanyang University, South Korea)

Deguang Kong (Google, USA)

Weize Kong (Google, USA)

Xiangnan Kong (Worcester Polytechnic Institute, USA)

Georgia Koutrika (Athena Research Center, Greece)

Elad Kravi (Amazon Research, Israel)
Ralf Krestel (Hasso Plattner Institute, University of

Potsdam, Germany, Germany)

Kun Kuang (Tsinghua University, China)

Anisio Lacerda (Centro Federal de Educação

Tecnológica de Minas Gerais, Brazil)

Wai Lam (The Chinese University of Hong Kong,

China)

Jean-Charles Lamirel (INRIA Project TALARIS -

LORIA, France)

Long Lan (National University of Defense

Technology, China)

Yanyan Lan (ICT, China)

Anne Laurent (LIRMM - UM, France)

Jae-Gil Lee (Korea Advanced Institute of Science \&

Technology, South Korea)

Wang-Chien Lee (The Pennsylvania State University, USA)

Chuan Lei (IBM Research-Almaden, USA)

Daniel Lemire (LICEF Research Center, Université du Québec, Canada)

Ulf Leser (Institut für Informatik, Humboldt-

Universität zu Berlin, Germany)

Ran Levy (Amazon, Israel)

Cheng-Te Li (National Cheng Kung University,

Taiwan)

Dongsheng Li (IBM Research - China, China)

Gang Li (Deakin University, Australia)

Hui Li (Xidian University, China)

Jianxin Li (Beihang University, China)

Jianxin Li (Deakin University, Australia)

Jing Li (Nanyang Technological University,

Singapore)

Jundong Li (University of Virginia, USA)

Kang Li (State University of New York at Buffalo, USA)

Ming Li (Nanjing University, China)

Peng Li (College of Information and Control

Engineering, China University of Petroleum (East

China), China)

Rong-Hua Li (Beijing Institute of Technology, China)

Shuai Li (University of Cambridge, UK)

Xiaoli Li (Institute for Infocomm Research, Singapore)

Ximing Li (Jilin university, China)

Xin Li (Beijing Institute of Technology, China)

Xinsheng Li (Apple, USA)

Xuelong Li (Chinese Academy of Sciences, China)

Yafei Li (Zhengzhou University, China) 
Yan Li (Alibaba Group, USA)

Yanhua Li (Worcester Polytechnic Institute (WPI), USA)

Yong Li (Tsinghua University, China)

Yuchen Li (Singapore Management University, Singapore)

Zechao Li (Nanjing University of Science \& Technology, China)

Zhu Li (Univ of Missouri, Kansas City, USA)

Hongwei Liang (Microsoft, Canada)

Qing Liao (Harbin Institute of Technology

(Shenzhen), China)

Alex Liu (Michigan State University, USA)

Baodi Liu (China University of Petroleum, China)

Bin Liu (IBM TJ Watson Research Center, USA)

Chien-Liang Liu (National Chiao Tung University, Taiwan)

Chuanren Liu (University of Tennessee, USA)

Guanfeng Liu (Macquarie University, Australia)

Hongfu Liu (Northeastern University, USA)

Hongyan Liu (Tsinghua University, China)

Junming Liu (City University of Hong Kong, Hong

Kong)

Ninghao Liu (Texas A\&M University, USA)

Qi Liu (University of Science \& Technology of China, China)

Wei Liu (University of Technology, Sydney, Australia)

Weifeng Liu (China University of Petroleum (East China), China)

Weiwei Liu (University of Technology, Sydney, Australia)

Xin Liu (The Hong Kong University of Science \& Technology, China)

Xinwang Liu (National University of Defense Technology, China)

Xuanzhe Liu (Peking University, China)

Yanchi Liu (Rutgers University, USA)

Cheng Long (Nanyang Technological University, Singapore)

Yin Lou (Ant Financial, USA)

Grigorios Loukides (King's College London, UK)

Can Lu (The Chinese University of Hong Kong, China)

Haibing Lu (Santa Clara University, USA)

Hua Lu (Aalborg University, Denmark)

Wei Lu (LinkedIn Corporation, USA)
Yi Lu (Massachusetts Institute of Technology, USA)

Claudio Lucchese (Ca' Foscari University of Venice, Italy)

Ping Luo (Institute of Computing Technology, CAS; University of Chinese Acadamy of Sciences, China)

Yong Luo (Nanyang Technological University, Singapore)

Guixiang Ma (Beijing Jiaotong University, USA)

Jianxin Ma (Tsinghua University, China)

Shuai Ma (Beihang University, China)

Sofian Maabout (LaBRI. University of Bordeaux, France)

Joel Mackenzie (RMIT University, Australia)

Masoud Makrehchi (University of Ontario Institute of Technology, Canada)

Tanu Malik (DePaul University, USA)

Brad Malin (Vanderbilt University, USA)

Hiroshi Mamitsuka (Kyoto University / Aalto

University, Japan)

Jiaxin Mao (Tsinghua University, China)

Brandeis Marshall (Spelman College, USA)

Wim Martens (University of Bayreuth, Germany)

Florent Masseglia (INRIA, France)

Ryan McBride (Simon Fraser University, Canada)

Parth Mehta (IRSI, India)

Xuying Meng (Chinese Academy of Sciences, China)

Paolo Merialdo (Università degli Studi Roma Tre,

Italy)

Pasquale Minervini (University of Bari, Italy)

Varun Mithal (LinkedIn, USA)

Boughanem Mohand (IRIT University Paul Sabatier

Toulouse, France)

Mitra Mohtarami (MIT Computer Science and

Artificial Intelligence Lab, USA)

Anna Monreale (University of Pisa, Italy)

Manuel Montes-Y-Gómez (Instituto Nacional de

Astrofísica, Óptica y Electrónica, Mexico)

Bongki Moon (Seoul National University, South

Korea)

Yang-Sae Moon (Kangwon National University, South Korea)

Felipe Moraes (Delft University of Technology, Netherlands)

Jose Moreno (IRIT/UPS, France)

Sebastian Moreno (Universidad Adolfo Ibañez, Chile)

Alessandro Moschitti (Amazon, USA) 
Heiko Mueller (New York University, USA)

Rene Mueller (NVIDIA, Switzerland)

Abhishek Mukherji (Cisco Systems, USA)

Tsuyoshi Murata (Tokyo Institute of Technology, Japan)

Filip Murlak (University of Warsaw, Poland)

Parth Nagarkar (New Mexico State University, USA)

Mirco Nanni (KDD-Lab ISTI-CNR Pisa, Italy)

Arun Reddy Nelakurthi (Samsung Electronics, USA)

Wilfred Ng (The Hong Kong University of Science \&

Technology, Hong Kong)

Ben C.K. Ngan (Worcester Polytechnic Institute, USA)

Feiping Nie (Northwestern Polytechnical University, China)

Jian-Yun Nie (University of Montreal, Canada)

Liqiang Nie (Shandong University, China)

Xia Ning (The Ohio State University, USA)

Werner Nutt (Free University of Bozen-Bolzano, Italy)

Tim Oates (University of Maryland Baltimore County, USA)

Beng Chin Ooi (National University of Singapore, Singapore)

Riccardo Ortale (ICAR-CNR, Italy)

Ming Pang (Nanjing University, China)

Spiros Papadimitriou (Rutgers University, USA)

Dhaval Patel (IBM, USA)

Torben Bach Pedersen (Aalborg University,

Denmark)

Hao Peng (Beihang University, China)

Wen-Chih Peng (National Chiao Tung University,

Taiwan)

Yun Peng (Lenovo, China)

Gustavo Penha (Delft University of Technology, Brazil)

Ruggero G. Pensa (University of Torino, Italy, Italy)

Jean-Marc Petit (Université de Lyon, INSA Lyon,

France)

Hai Phan (New Jersey Institute of Technology, USA)

Gianvito Pio (University of Bari “Aldo Moro”, Italy)

Silvestro Roberto Poccia (university of Turin, Italy)

Tieyun Qian (Wuhan University, China)

Yanan Qian (Xi'an Jiaotong University, China)

Maoying Qiao (University of Technology, Sydney, Australia)
Miao Qiao (The University of Auckland, New Zealand)

Lu Qin (University of Technology, Sydney, Australia)

Xiao Qin (IBM, USA)

Minghui Qiu (Alibaba, Singapore)

Ganesh Ramesh (Yahoo Inc., USA)

Jan Ramon (INRIA, France)

Louiqa Raschid (University of Maryland, USA)

Chedy Raïssi (INRIA, France)

Zhaochun Ren (JD.com, China)

Matteo Riondato (Amherst College, USA)

Tore Risch (Uppsala University, Sweden)

Carolina Ruiz (WPI, USA)

Altigran S. Da Silva (Universidade Federal do Amazonas, Brazil)

Lorenza Saitta (Universita del Piemonte Orientale, Italy)

Pierangela Samarati (University of Milan, Italy)

Kameshwaran Sampath (IBM India Research Lab, India)

David Sanchez (University Rovira i Virgili, Spain)

Ognjen Savkovic (Free University of Bolzano, Italy)

Pavel Senin (Los Alamos National Laboratory, USA)

Vinay Setty (University of Stavanger, Norway)

Azadeh Shakery (University of Tehran, Iran)

Ming Shao (UMass Dartmouth, USA)

Li Shen (Tencent AI Lab, China)

Wei Shen (Nankai University, China)

Xiaobo Shen (Nanjing University of Science \&

Technology, China)

Yanyan Shen (Shanghai Jiao Tong University, China)

Zheyan Shen (Tsinghua University, China)

Chuan Shi (Beijing University of Posts and

Telecommunications, China)

Jieming Shi (National University of Singapore, Singapore)

Kyuseok Shim (Seoul National University, South

Korea)

Gianmaria Silvello (University of Padua, Italy)

Dongjin Song (NEC Labs America, USA)

Shaoxu Song (Tsinghua University, China)

Damiano Spina (RMIT University, Australia)

Anna Squicciarini (The Pennsylvania State

University, USA)

Ja-Hwung Su (NCKU, Taiwan)

Zhiyang Su (The Hong Kong University of Science \& Technology, Hong Kong) 
Guangzhong Sun (University of Science \&

Technology of China, China)

Yizhou Sun (University of California, Los Angeles, USA)

Zhu Sun (Nanyang Technological University, Singapore)

Chih-Hua Tai (National Taiwan University, Taiwan)

Atsuhiro Takasu (National Institute of Informatics, Japan)

Letizia Tanca (Politecnico di Milano, Italy)

Jiaxi Tang (Simon Fraser University, Canada)

Jiliang Tang (Michigan State University, USA)

David Taniar (Monash University, Australia)

Masahiro Terabe (Chugai Pharmaceutical Co., Ltd., Japan)

Feng Tian (Jiaotong University, China)

Jin Ting (Hainan University, China)

Nicola Tonellotto (ISTI-CNR, Italy)

Bin Tong (Central Research Lab., Hitachi, Japan)

Vicenc Torra (U. Skövde, Sweden)

Goce Trajcevski (Iowa State University, USA)

Shusaku Tsumoto (Shimane University, Japan)

$\mathrm{Ke} \mathrm{Tu}$ (Tsinghua University, China)

Aparna Varde (Montclair State University, USA)

Hannes Voigt (Neo4j, Germany)

Slobodan Vucetic (Temple University, USA)

Alice Wang (Spotify, USA)

Chang-Dong Wang (Sun Yat-sen University, China)

Chaokun Wang (Tsinghua University, China)

Chonggang Wang (InterDigital Communications, USA)

Daheng Wang (University of Notre Dame, USA)

Daixin Wang (Antfin Financial Services Group, China, China)

Dong Wang (Dalian University of Technology, China)

Honggang Wang (University of Massachusetts, USA)

Hongjian Wang (Twitter Inc., USA)

Jianyong Wang (Tsinghua University, China)

Jin Wang (University of California, Los Angeles, USA)

Jingyuan Wang (Beihang University, China)

Nannan Wang (Xidian University, China)

Sen Wang (School of Information Technology and Electrical Engineering, Australia)

Sibo Wang (The Chinese University of Hong Kong, Hong Kong)
Suhang Wang (The Pennsylvania State University, USA)

Ting Wang (Lehigh University, USA)

Weiqing Wang (Monash University, Australia)

Xiang Wang (National University of Singapore,

Singapore)

Xiao Wang (Beijing University of Posts and

Telecommunications, China)

Xiaoling Wang (ECNU, China)

Xiaoyang Wang (Zhejiang Gongshang University, China)

Xin Wang (Tsinghua University, China)

Yangang Wang (Southeast University, China)

Yongqing Wang (Institute of Automation, Chinese

Academy of Sciences, China)

Zhengyang Wang (Texas A\&M University, USA)

Zhi Wang (Tsinghua University, China)

Ingmar Weber (Qatar Computing Research Institute, Qatar)

Hao Wei (Amazon, USA)

Wei Wei (Huazhong University of Science and

Techonology, China)

Zhi Wei (NJIT, USA)

Gerhard Weikum (Max Planck Institute for

Informatics, Germany)

Dong Wen (University of Technology, Sydney, Australia)

Zeyi Wen (National University of Singapore,

Singapore)

Jianshu Weng (Swiss Re, Singapore)

Raymond Chi-Wing Wong (The Hong Kong

University of Science \& Technology, Hong Kong)

Peter Wood (Birkbeck, University of London, UK)

Chuan $\mathrm{Wu}$ (The University of Hong Kong, Hong

Kong)

Fei Wu (The Pennsylvania State University, USA)

Jia Wu (Macquarie University, Australia)

Le $\mathrm{Wu}$ (Hefei University of Technology, China)

Liang Wu (Airbnb, Inc., USA)

Lingfei Wu (IBM Research AI, USA)

Sai Wu (Zhejiang University, China)

Shan-Hung Wu (National Tsing Hua University,

Taiwan)

Yinghui Wu (Washington State University, USA)

Yulei $\mathrm{Wu}$ (UOE, UK)

Feng Xia (Dalian University of Technology, China)

Yinglong Xia (Futurewei Technologies, USA) 
Lingyun Xiang (Changsha University of Science \&

Technology, China)

Houping Xiao (Georgia State University, USA)

Keli Xiao (Stony Brook University, USA)

Wenyi Xiao (The Hong Kong University of Science \&

Technology, Hong Kong)

Yanghua Xiao (Fudan University, China)

Sihong Xie (Lehigh University, USA)

Sihong Xie (Lehigh University, USA)

Xiaohui Xie (Tsinghua University, China)

Yun Xiong (Fudan University, China)

Chang Xu (The University of Sydney, Australia)

Jian Xu (Alibaba Group, USA)

Jun Xu (Renmin University of China, China)

Zhao Xu (NEC Laboratories Europe, Germany)

Takehiro Yamamoto (University of Hyogo, Japan)

Rui Yan (Peking University, China)

Xiao Yan (LinkedIn, USA)

Bin Yang (Aalborg University, Denmark)

Bo Yang (Jilin University, China)

Hui Yang (san francisco state university, USA)

Qing Yang (University of North Texas, USA)

Yu Yang (City University of Hong Kong, Hong Kong)

Huaxiu Yao (The Pennsylvania State University, USA)

Lina Yao (The University of New South Wales,

Australia)

Yibo Yao (Washington State University, USA)

Yuan Yao (Nanjing University, China)

Zijun Yao (IBM, USA)

Yanfang Ye (West Virginia University, USA)

Xu Yi (Beihang University, China)

Dawei Yin (JD.com, USA)

Hongzhi Yin (The University of Queensland, Australia)

Dantong Yu (New Jersey Institute of Technology, USA)

Guoxian Yu (Southwest University, China, China)

Hai-Tao Yu (University of Tsukuba, UK)

Linyun Yu (Bytedance Inc, China)

Ting Yu (Qatar Computing Research Institute, Qatar)

Yang Yu (Nanjing University, China)

Yanwei Yu (Yantai University, China)

Zhou Yu (Hangzhou Dianzi University, China)

Long Yuan (Nanjing University of Science \&

Technology, China)

Qiangqiang Yuan (wuhan university, China)

Ye Yuan (NEU, China)
Reza Zafarani (Syracuse University, USA)

Hamed Zamani (University of Massachusetts Amherst, USA)

Chengxi Zang (Tsinghua University --> Cornell University, USA)

Chunqiu Zeng (Google, USA)

Ziqian Zeng (The Hong Kong University of Science \& Technology, Hong Kong)

Aidong Zhang (University of Virginia, USA)

Chao Zhang (Georgia Institute of Technology, USA)

Chao Zhang (Dalian University of Technology, China)

Chenwei Zhang (Amazon, USA)

Chuxu Zhang (University of Notre Dame, USA)

Dan Zhang (Facebook, USA)

Dongxiang Zhang (Zhejiang University, China)

Fan Zhang (Guangzhou University, China)

Hongming Zhang (The Hong Kong University of

Science \& Technology, Hong Kong)

Jiawei Zhang (Florida State University, USA)

Junqi Zhang (Tsinghua University, China)

Kun Zhang (Xavier University of Louisiana, USA)

Lefei Zhang (Wuhan University, China)

Min Zhang (Tsinghua University, China)

Qi Zhang (Fudan University, China)

Richong Zhang (Beihang University, China)

Shichao Zhang (Guangxi Normal University, China)

Shuhao Zhang (National University of Singapore,

Singapore)

Si Zhang (University of Illinois Urbana-Champaign, USA)

Wei Zhang (East China Normal University, China)

Wei Emma Zhang (The University of Adelaide,

Australia)

Weinan Zhang (Shanghai Jiao Tong University, China)

Weizhong Zhang (Zhejiang University, China)

Wenlu Zhang (California State University Long Beach, USA)

Xiang Zhang (The Pennsylvania State University, USA)

Xiangliang Zhang (King Abdullah University of

Science \& Technology, Saudi Arabia)

Xiaoying Zhang (The Chinese University of Hong

Kong, Hong Kong)

Xiuzhen Zhang (RMIT University, Australia) 
Xuyun Zhang (The University of Auckland, New Zealand)

Ya Zhang (Shanghai Jiao Tong University, China)

Yanchun Zhang (Victoria University, Australia)

Yong Zhang (Huawei Technologies Canada Co., Ltd., Canada)

Zhao Zhang (Hefei University of Technology, China)

Zhenjie Zhang (LinkedIn, USA)

Zhiwei Zhang (Hong Kong Baptist University, Hong Kong)

Ziwei Zhang (Tsinghua University, China)

Haifeng Zhao (Linkedin, USA)

Peixiang Zhao (Florida State University, USA)

Shiwan Zhao (IBM Research - China, China)

Xiang Zhao (National University of Defense Technology, China)

Xin Zhao (Renmin University of China, China)

Ying Zhao (Tsinghua University, China)

Yuhai Zhao (northeastern university, China)

Baihua Zheng (none, Singapore)

Guanjie Zheng (The Pennsylvania State University, USA)

Kai Zheng ("University of Electronic Science \& Technology of China”, China)

Weiguo Zheng (Fudan University, China)

Yudian Zheng (Twitter, USA)

Yukun Zheng (Tsinghua University, China)
Ning Zhong (Maebashi Institute of Technology, China)

Amelie Chi Zhou (Shenzhen University, China)

Aoying Zhou (East China Normal University, China)

Bin Zhou (University of Maryland, Baltimore County, USA)

Dawei Zhou (University of Illinois at UrbanaChampaign, USA)

Fang Zhou (East China Normal University, China)

Xiangmin Zhou (RMIT University, Australia)

Xin Zhou (Nanyang Technological University, Singapore)

Xun Zhou (University of Iowa, USA)

Yang Zhou (Auburn University, USA)

Yao Zhou (University of Illinois at UrbanaChampaign, USA)

Hengshu Zhu (Baidu Inc., China)

Lin Zhu (Linkedin, USA)

Xingquan Zhu (Florida Atlantic University, USA)

Yuanyuan Zhu (Wuhan University, China)

Fuzhen Zhuang (Institute of Computing Technology, Chinese Academy of Sciences, China)

Yong Zhuang (University of Massachussets Boston, USA)

Zhongfang Zhuang (Visa Research, USA)

Imed Zitouni (Microsoft, USA)

Justin Zobel (The University of Melbourne, Australia)

Jia Zou (Arizona State University, USA) 


\section{CIKM'19 Program Committee Members - Short Track}

Ziawasch Abedjan (TU Berlin, Germany)

Karl Aberer (Ecole Polytechnique Fédérale de

Lausanne, Switzerland)

Ayan Acharya (CognitiveScale Inc., USA)

Ashvin Agrawal (Microsoft, USA)

Ismail Altingovde (Middle East Technical University,

Turkey)

Giambattista Amati (Fondazione Ugo Bordoni, Italy)

Enrique Amigó (UNED, Spain)

Avi Arampatzis (Democritus University of Thrace,

Greece)

Walid Aref (Purdue University, USA)

Renato Assuncao (UFMG, Brazil)

Hosein Azarbonyad (University of Amsterdam,

Netherlands)

Xiao Bai (Yahoo Research, USA)

Leandro Marinho (Federal University of Campina

Grande (UFCG), Brazil)

Peng Bao (Beijing Jiaotong University, China)

Senjuti Roy (NJIT, USA)

Roberto Bayardo (Google, USA)

Ryan Benton (University of South Alabama, USA)

Michele Berlingerio (IBM, Ireland)

Elisa Bertino (Purdue University, USA)

Sumit Bhatia (IBM, India)

Siddhartha Bhattacharyya (University of Illinois,

Chicago, USA)

Jiang Bian (Microsoft, China)

Asia Biega (Microsoft, Canada)

Bodo Billerbeck (Bing, Australia)

Francesco Bonchi (Fondazione ISI, Italy)

Ilaria Bordino (UniCredit R\&D, Italy)

Renata Borovica-Gajic (The University of Melbourne, Australia)

Marco Brambilla (Politecnico di Milano, Italy)

Pavel Braslavski (Ural Federal University, Russia)

Ivan Brugere (Salesforce Research, USA)

François Bry (Ludwig Maximilian University of Munich, Germany)

Yingyi Bu (Google, USA)

Klemens Böhm (Karlsruhe Institute of Technology, Germany)

Fidel Cacheda (Universidade da Coruña, Spain)
Jose Cadena (Lawrence Livermore National Laboratory, USA)

Xiangrui Cai (Nankai University, China)

Zhipeng Cai (Georgia State University, USA)

Fazli Can (Bilkent University, Turkey)

Huiping Cao (New Mexico State University, USA)

Jian Cao (Shanghai Jiao Tong University, China)

Lei Cao (Massachusetts Institute of Technology, USA)

Longbing Cao (Faculty of IT, University of Technology

Sydney, Australia)

Xin Cao (The University of New South Wales,

Australia)

Yang Cao (The University of Edinburgh, UK)

Bogdan Cautis (ENST Paris - UMR CNRS 5141, France)

James Caverlee (Texas A\&M University, USA)

Michelangelo Ceci (Universita degli Studi di Bari, Italy)

Tanmoy Chakraborty (IIIT-Delhi, India, India)

Sharma Chakravarthy (The University of Texas at

Arlington, USA)

Jeffrey Chan (RMIT University, Australia)

Keith Chan (The Hong Kong Polytechnic University,

Hong Kong)

Philip Chan (Florida Institute of Technology, USA)

Praveen Chandar (Spotify Research, USA)

Kevin Chang (University of Illinois at Urbana-

Champaign, USA)

Abon Chaudhuri (WalmartLabs, USA)

Muhammad Cheema (Monash University, Australia)

Chen Chen (Arizona State University, USA)

Feng Chen (University at Albany - SUNY, USA)

Hsin-Hsi Chen (National Taiwan University, Taiwan)

Huanhuan Chen (University of Science \& Technology of China, China)

Huiyuan Chen (Case Western Reserve University, USA)

Jinjun Chen (University of Technology, Sydney, Australia)

Ling Chen (University of Technology, Sydney, Australia)

Lisi Chen (Hong Kong Baptist University, Hong Kong)

Ping Chen (UMB, USA)

Qian Chen (Google, China)

Ruey-Cheng Chen (SEEK Ltd, Australia)

Rui Chen (Samsung Electronics, USA) 
Shu-Ching Chen (Florida International University, USA)

Xilun Chen (Arizona State University, USA)

$\mathrm{Xu}$ Chen (Tsinghua University, China)

Yang Chen (LinkedIn, USA)

Zhengzhang Chen (NEC Laboratories America, USA)

Zhenzhong Chen (Wuhan University, China)

Zhiyuan Chen (University of Maryland Baltimore County, USA)

Zhumin Chen (shandong university, China)

Dawei Cheng (Shanghai Jiao Tong University, China)

Yurong Cheng (Beijing Institute of Technology, China)

Fei Chiang (McMaster University, Canada)

Adrian-Gabriel Chifu (Aix Marseille Univ, Université de Toulon, CNRS, LIS, France)

Silvia Chiusano (Politecnico di Torino, Italy)

Vassilis Christophides (INRIA, France)

Panos Chrysanthis (University of Pittsburgh, USA)

Lingyang Chu (Simon Fraser University, Canada)

Korris Chung (The Hong Kong Polytechnic University, China)

Arman Cohan (Allen Institute for Artificial Intelligence (AI2), USA)

Nachshon Cohen (Ecole Polytechnique Fédérale de Lausanne, Switzerland)

Michael Cole (LexisNexis, USA)

Gao Cong (Nanyang Technological University, Singapore)

Michele Coscia (IT University of Copenhagen, Denmark)

Matt Crane (Facebook, USA)

Bruno Cremilleux (Universite de Caen Normandie, France)

Paolo Cremonesi (Politecnico di Milano, Italy)

Philippe Cudre-Mauroux (U. of Fribourg, Switzerland)

Tonya Custis (Thomson Reuters, USA)

Alfredo Cuzzocrea (ICAR-CNR and University of Calabria, Italy)

Arthur Câmara (Delft University of Technology, Netherlands)

Hong-Ning Dai (Macau Univ. of Sci. and Tech., Macao)

Mahashweta Das (Visa Research, USA)

Anwitaman Datta (Nanyang Technological University, Singapore)

Edleno Silva De Moura (Universidade Federal do Amazonas, Brazil)

Maarten Rijke (University of Amsterdam, Netherlands)
Cheng Deng (Xidian University, China)

Shuiguang Deng (Zhejiang University, China)

Bolin Ding (Alibaba Group, USA)

Jingtao Ding (Tsinghua University, Beijing, P.R.China, China)

Yao-Xiang Ding (Nanjing University, China)

Ying Ding (Indiana University Bloomington, USA)

Nemanja Djuric (Uber ATG, USA)

Josep Domingo-Ferrer (Universitat Rovira i Virgili, Spain)

Yuxiao Dong (Microsoft, USA)

Dejing Dou (Computer and Information Science

Department, University of Oregon, USA)

Zhicheng Dou (Renmin University of China, China)

Peng Du (LinkedIn Corporation, USA)

Tamer Elsayed (Qatar University, Qatar)

Mohamed Eltabakh (WPI, USA)

Vlad Estivill-Castro (Griffith University, Australia)

Xuhui Fan (UTS, Australia)

Yi Fang (Santa Clara University, USA)

Yixiang Fang (The University of New South Wales,

Australia)

Yuan Fang (Singapore Management University,

Singapore)

Fuli Feng (National University of Singapore, Singapore)

Qingxiang Feng (Tulane University, USA)

Yunlong Feng (SUNY Albany, USA)

Hakan Ferhatosmanoglu (The University of Warwick, UK)

Elena Ferrari (University of Insubria, Italy)

Donatella Firmani (Roma Tre University, Italy)

Peter Fischer (Universität Augsburg, Institut für

Informatik, Germany)

George Fletcher (Eindhoven University of Technology,

Netherlands)

Edward Fox (Virginia Tech, USA)

Marios Fragkoulis (AUEB, Greece)

Yanjie Fu (University of Central Florida, USA)

Luke Gallagher (RMIT University, Australia)

Junhao Gan (The University of Melbourne, Australia)

Byron Gao (Texas State University, USA)

Chen Gao (Tsinghua University, China)

Jun Gao (none, China)

Min Gao (Chongqing University, China)

Yue Gao (Tsinghua University, China)

Yash Garg (Arizona State University, USA) 
Wolfgang Gatterbauer (Khoury College of Computer Sciences, Northeastern University, USA)

Tingjian Ge (University of Massachusetts, Lowell, USA)

Dimitrios Georgakopoulos (Swinburne University of Technology, Australia)

Sahin Geyik (LinkedIn, USA)

Aris Gkoulalas-Divanis (IBM Watson Health, Cambridge, MA, USA, USA)

Boris Glavic (Illinois Institute of Technology, USA)

Marcos Goncalves (Federal University of Minas Gerais, Brazil)

Dihong Gong (UF, USA)

Mingming Gong (University of Pittsburgh, USA)

Julio Gonzalo (UNED, Spain)

Sergio Greco (University of Calabria, Italy)

Ziyu Guan (Northwest University of China, China)

Jie Gui (hfcas, China)

Francesco Gullo (UniCredit, Italy)

Qi Guo (LinkedIn, USA)

Parth Gupta (Amazon, Spain)

Jialong Han (Nanyang Technological University, Singapore)

Qilong Han (College of Computer Science \& Technology,Harbin Engineering University, China)

Ben He (University of Chinese Academy of Sciences, China)

Dongxiao He (School of Computer Science \& Technology, Tianjin University, China)

Jingrui He (University of Illinois at Urbana-Champaign, USA)

Jiong He (Alibaba Group, Singapore)

Xiaofeng He (East China Normal University, China)

Yeye He (Microsoft, USA)

Zhenying He (fudan university, China)

Sven Helmer (University of Zurich, Switzerland)

Heli Helskyaho (Miracle Finland Oy, Finland)

Shen-Shyang Ho (Rowan University, USA)

Tuan-Anh Hoang (L3S Research Center, Leibniz

University of Hanover, Germany)

Liangjie Hong (Etsy Inc., USA)

Frank Hopfgartner (The University of Sheffield, UK)

Bill Howe (, USA)

$\mathrm{Han} \mathrm{Hu}$ (Beijing Institute of Technology, China, China) Juhua $\mathrm{Hu}$ (University of Washington | Tacoma, USA)

Ziniu Hu (University of California, Los Angeles, USA)

Kaixun Hua (University of Massachusetts Boston, USA)

Wen Hua (The University of Queensland, Australia)
Chao Huang (University of Notre Dame, USA)

Jin Huang (Tsinghua University, China)

Joshua Huang (Shenzhen University, China)

Wei Huang (State Key Lab of Software

Engineering,Wuhan university, Wuhan 430072, China)

Xiao Huang (Texas A\&M University, USA)

Xin Huang (Hong Kong Baptist University, Hong

Kong)

Xuanjing Huang (Fudan University, China)

Chih-Chieh Hung (Tamkang University, Taiwan)

Tsuyoshi Ide (IBM, USA)

Sam Idicula (Oracle, USA)

Amir Ingber (Amazon Research, Israel)

Stratis Ioannidis (Northeastern University, USA)

Radu Ionescu (University of Bucharest, Faculty of

Mathematics and Computer Science, Romania)

Raihanul Islam (Virginia Tech, USA)

Feng Ji (DAMO Academy, Alibaba Group, China)

Ling Jian (China University of Petroleum, China)

Daxin Jiang (Microsoft, China)

Di Jiang (WeBank, China)

Wenhao Jiang (Tencent, China)

Bo Jin (Dalian University of Technology, China)

Di Jin (Tianjin University, China)

Ruoming Jin (Kent State University, USA)

Alekh Jindal (Microsoft, USA)

Gareth Jones (Dublin City University, Ireland)

Roy Lee (Singapore Management University,

Singapore)

Panos Kalnis (King Abdullah University of Science \&

Technology, Saudi Arabia)

Konstantinos Kalpakis (UMBC, USA)

Bhargav Kanagal (Google, USA)

U Kang (Seoul National University, South Korea)

Zhao Kang (southern illinois university carbondale, USA)

Verena Kantere (University of Ottawa, Canada)

Ben Kao (The University of Hong Kong, Hong Kong)

Zoi Kaoudi (Qatar Computing Research Institute, Qatar)

Kamalakar Karlapalem (CDE, IIIT Hyderabad, India, India)

Panagiotis Karras (Aarhus University, Denmark)

Harshad Kasture (Oracle Labs, USA)

Makoto Kato (University of Tsukuba, Japan)

Tom Kenter (University of Amsterdam, Netherlands) 
Eamonn Keogh (UC Riverside, USA)

Maurice Keulen (University of Twente, Netherlands)

Arijit Khan (Nanyang Technological University,

Singapore)

Myunghwan Kim (LinkedIn Corporation, USA)

Sang-Wook Kim (Hanyang University, South Korea)

Deguang Kong (Google, USA)

Weize Kong (Google, USA)

Xiangnan Kong (Worcester Polytechnic Institute, USA)

Georgia Koutrika (Athena Research Center, Greece)

Elad Kravi (Amazon Research, Israel)

Ralf Krestel (Hasso Plattner Institute, University of

Potsdam, Germany, Germany)

Kun Kuang (Tsinghua University, China)

Anisio Lacerda (Centro Federal de Educação

Tecnológica de Minas Gerais, Brazil)

Wai Lam (The Chinese University of Hong Kong, China)

Jean-Charles Lamirel (INRIA Project TALARIS LORIA, France)

Long Lan (national university of defense technology, China)

Yanyan Lan (ICT, China)

Anne Laurent (LIRMM - UM, France)

Jae-Gil Lee (Korea Advanced Institute of Science \&

Technology, South Korea)

Wang-Chien Lee (The Pennsylvania State University, USA)

Chuan Lei (IBM Research-Almaden, USA)

Daniel Lemire (LICEF Research Center, Université du Québec, Canada)

Ulf Leser (Institut für Informatik, Humboldt-

Universität zu Berlin, Germany)

Ran Levy (Amazon, Israel)

Cheng-Te Li (National Cheng Kung University, Taiwan)

Dongsheng Li (IBM Research - China, China)

Gang Li (Deakin University, Australia)

Hui Li (Xidian University, China)

Jianxin Li (Beihang University, China)

Jianxin Li (Deakin University, Australia)

Jing Li (Nanyang Technological University, Singapore)

Jundong Li (University of Virginia, USA)

Kang Li (State University of New York at Buffalo, USA)

Ming Li (Nanjing University, China)

Peng Li (China University of Petroleum (East China),

China)
Rong-Hua Li (Beijing Institute of Technology, China)

Shuai Li (University of Cambridge, UK)

Xiaoli Li (Institute for Infocomm Research, Singapore)

Ximing Li (Jilin university, China)

Xin Li (Beijing Institute of Technology, China)

Xinsheng Li (Apple, USA)

Xuelong Li (Chinese Academy Of Sciences, China)

Yafei Li (Zhengzhou University, China)

Yan Li (Alibaba Group, USA)

Yanhua Li (Worcester Polytechnic Institute (WPI), USA)

Yong Li (Tsinghua University, China)

Yuchen Li (Singapore Management University, Singapore)

Zechao Li (Nanjing University of Science \&

Technology, China)

Zhu Li (Univ of Missouri, Kansas City, USA)

Hongwei Liang (Microsoft, Canada)

Qing Liao (Harbin Institute of Technology (Shenzhen), China)

Alex Liu (Michigan State University, USA)

Baodi Liu (China University of Petroleum, China)

Bin Liu (IBM TJ Watson Research Center, USA)

Chien-Liang Liu (National Chiao Tung University, Taiwan)

Chuanren Liu (University of Tennessee, USA)

Guanfeng Liu (Macquarie University, Australia)

Hongfu Liu (Northeastern University, USA)

Hongyan Liu (Tsinghua University, China)

Junming Liu (City University of Hong Kong, Hong

Kong)

Ninghao Liu (Texas A\&M University, USA)

Qi Liu (University of Science \& Technology of China, China)

Wei Liu (University of Technology, Sydney, Australia)

Weifeng Liu (China University of Petroleum (East China), China)

Weiwei Liu (University of Technology, Sydney, Australia)

Xinwang Liu (School of Computer, National University of Defense Technology, China)

Xuanzhe Liu (Peking University, China)

Yanchi Liu (Rutgers University, USA)

Cheng Long (Nanyang Technological University, Singapore)

Yin Lou (Ant Financial, USA)

Grigorios Loukides (King's College London, UK) 
Can Lu (The Chinese University of Hong Kong, China)

Haibing Lu (Santa Clara University, USA)

Hua Lu (Aalborg University, Denmark)

Wei Lu (LinkedIn Corporation, USA)

Yi Lu (Massachusetts Institute of Technology, USA)

Claudio Lucchese (Ca' Foscari University of Venice, Italy)

Ping Luo (Institute of Computing Technology, CAS; University of Chinese Acadamy of Sciences, China)

Yong Luo (Nanyang Technological University, Singapore)

Guixiang Ma (Univ, USA)

Jianxin Ma (Tsinghua University, China)

Shuai Ma (Beihang University, China)

Sofian Maabout (LaBRI. University of Bordeaux, France)

Joel Mackenzie (RMIT University, Australia)

Masoud Makrehchi (University of Ontario Institute of Technology, Canada)

Tanu Malik (DePaul University, USA)

Brad Malin (Vanderbilt University, USA)

Hiroshi Mamitsuka (Kyoto University / Aalto

University, Japan)

Jiaxin Mao (Tsinghua University, China)

Brandeis Marshall (Spelman College, USA)

Wim Martens (University of Bayreuth, Germany)

Florent Masseglia (INRIA, France)

Ryan McBride (Simon Fraser University, Canada)

Parth Mehta (IRSI, India)

Xuying Meng (Chinese Academy of Sciences, China)

Paolo Merialdo (Università degli Studi Roma Tre, Italy)

Pasquale Minervini (University of Bari, Italy)

Varun Mithal (LinkedIn corporation, USA)

Boughanem Mohand (IRIT University Paul Sabatier

Toulouse, France)

Mitra Mohtarami (MIT Computer Science and Artificial Intelligence Lab, USA)

Anna Monreale (University of Pisa, Italy)

Manuel Montes-Y-Gómez (Instituto Nacional de Astrofísica, Óptica y Electrónica, Mexico)

Bongki Moon (Seoul National University, South Korea)

Yang-Sae Moon (Kangwon National University, South Korea)

Felipe Moraes (Delft University of Technology, Netherlands)

Jose Moreno (IRIT/UPS, France)

Sebastian Moreno (Universidad Adolfo Ibañez, Chile)
Alessandro Moschitti (Amazon, USA)

Heiko Mueller (New York University, USA)

Rene Mueller (NVIDIA, Switzerland)

Abhishek Mukherji (Cisco Systems, USA)

Tsuyoshi Murata (Tokyo Institute of Technology, Japan)

Filip Murlak (University of Warsaw, Poland)

Parth Nagarkar (New Mexico State University, USA)

Mirco Nanni (KDD-Lab ISTI-CNR Pisa, Italy)

Arun Reddy Nelakurthi (Samsung Electronics, USA)

Wilfred Ng (The Hong Kong University of Science \&

Technology, Hong Kong)

Ben Ngan (Worcester Polytechnic Institute, USA)

Feiping Nie (Northwestern Polytechnical University, China)

Jian-Yun Nie (University of Montreal, Canada)

Liqiang Nie (Shandong University, China)

Werner Nutt (Free University of Bozen-Bolzano, Italy)

Tim Oates (University of Maryland Baltimore County, USA)

Beng Ooi (National University of Singapore, Singapore)

Riccardo Ortale (ICAR-CNR, Italy)

Ming Pang (Nanjing University, China)

Spiros Papadimitriou (Rutgers University, USA)

Dhaval Patel (IBM, USA)

Torben Pedersen (Aalborg University, Denmark)

Wen-Chih Peng (National Chiao Tung University,

Taiwan)

Yun Peng (Lenovo, China)

Gustavo Penha (Delft University of Technology, Brazil)

Ruggero Pensa (University of Torino, Italy, Italy)

Jean-Marc Petit (Université de Lyon, INSA Lyon,

France)

Hai Phan (New Jersey Institute of Technology, USA)

Gianvito Pio (University of Bari "Aldo Moro", Italy)

Silvestro Poccia (university of Turin, Italy)

Tieyun Qian (Wuhan University, China)

Yanan Qian (Xi'an Jiaotong University, China)

Maoying Qiao (University of Technology, Sydney, Australia)

Miao Qiao (The University of Auckland, New Zealand)

Lu Qin (University of Technology, Sydney, Australia)

Xiao Qin (IBM, USA)

Minghui Qiu (Alibaba, Singapore)

Ganesh Ramesh (Yahoo Inc., USA)

Jan Ramon (INRIA, France)

Louiqa Raschid (University of Maryland, USA) 
Chedy Raïssi (INRIA, France)

Zhaochun Ren (JD.com, China)

Matteo Riondato (Amherst College, USA)

Tore Risch (Uppsala University, Sweden)

Carolina Ruiz (WPI, USA)

Altigran Silva (Universidade Federal do Amazonas, Brazil)

Lorenza Saitta (Universita del Piemonte Orientale, Italy)

Pierangela Samarati (University of Milan, Italy)

Kameshwaran Sampath (IBM India Research Lab, India)

David Sanchez (University Rovira i Virgili, Spain)

Ognjen Savkovic (Free University of Bolzano, Italy)

Pavel Senin (Los Alamos National Laboratory, USA)

Vinay Setty (University of Stavanger, Norway)

Azadeh Shakery (University of Tehran, Iran)

Ming Shao (UMass Dartmouth, USA)

Li Shen (Tencent AI Lab, China)

Wei Shen (Nankai University, China)

Xiaobo Shen (Nanjing University of Science \&

Technology, China)

Yanyan Shen (Shanghai Jiao Tong University, China)

Zheyan Shen (Tsinghua University, China)

Chuan Shi (Beijing University of Posts and

Telecommunications, China)

Jieming Shi (National University of Singapore, Singapore)

Kyuseok Shim (Seoul National University, South

Korea)

Gianmaria Silvello (University of Padua, Italy)

Dongjin Song (NEC Labs America, USA)

Shaoxu Song (Tsinghua University, China)

Damiano Spina (RMIT University, Australia)

Anna Squicciarini (The Pennsylvania State University, USA)

Ja-Hwung Su (NCKU, Taiwan)

Zhiyang Su (The Hong Kong University of Science \& Technology, Hong Kong)

Guangzhong Sun (University of Science \& Technology of China, China)

Yizhou Sun (University of California, Los Angeles, USA)

Zhu Sun (Nanyang Technological University, Singapore)

Chih-Hua Tai (National Taiwan University, Taiwan)
Atsuhiro Takasu (National Institute of Informatics, Japan)

Letizia Tanca (Politecnico di Milano, Italy)

Jiaxi Tang (Simon Fraser University, Canada)

Jiliang Tang (Michigan State University, USA)

David Taniar (Monash University, Australia)

Masahiro Terabe (Chugai Pharmaceutical Co., Ltd., Japan)

Feng Tian (Xi'an Jiaotong University, China)

Jin Ting (Hainan University, China)

Nicola Tonellotto (ISTI-CNR, Italy)

Bin Tong (Central Research Lab., Hitachi, Japan)

Vicenc Torra (U. Skövde, Sweden)

Goce Trajcevski (Iowa State University, USA)

Shusaku Tsumoto (Shimane University, Japan)

Ke Tu (Tsinghua University, China)

Aparna Varde (Montclair State University, USA)

Hannes Voigt (Neo4j, Germany)

Slobodan Vucetic (Temple University, USA)

Alice Wang (Spotify, USA)

Chang-Dong Wang (Sun Yat-sen University, China)

Chaokun Wang (Tsinghua University, China)

Chonggang Wang (InterDigital Communications, USA)

Daheng Wang (University of Notre Dame, USA)

Daixin Wang (Antfin Financial Services Group, China, China)

Dong Wang (Dalian University of Technology, China)

Honggang Wang (University of Massachusetts, USA)

Hongjian Wang (Twitter Inc., USA)

Jianyong Wang (Tsinghua University, China)

Jin Wang (University of California, Los Angeles, USA)

Jingyuan Wang (Beihang University, China)

Nannan Wang (Xidian University, China)

Sibo Wang (The Chinese University of Hong Kong, Hong Kong)

Suhang Wang (The Pennsylvania State University, USA)

Ting Wang (Lehigh University, USA)

Weiqing Wang (Monash University, Australia)

Xiang Wang (National University of Singapore, Singapore)

Xiao Wang (Beijing University of Posts and

Telecommunications, China)

Xiaoling Wang (ECNU, China)

Xiaoyang Wang (Zhejiang Gongshang University, China)

Xin Wang (Tsinghua University, China) 
Yangang Wang (, China)

Yongqing Wang (Institute of Automation, Chinese Academy of Sciences, China)

Zhengyang Wang (Texas A\&M University, USA)

Zhi Wang (Tsinghua University, China)

Ingmar Weber (Qatar Computing Research Institute, Qatar)

Hao Wei (Amazon, USA)

Wei Wei (Huazhong University of Science and

Techonology, China)

Zhi Wei (NJIT, USA)

Gerhard Weikum (Max Planck Institute for Informatics, Germany)

Dong Wen (University of Technology, Sydney, Australia)

Zeyi Wen (National University of Singapore, Singapore)

Jianshu Weng (Swiss Re, Singapore)

Raymond Wong (The Hong Kong University of Science \& Technology, Hong Kong)

Peter Wood (Birkbeck, University of London, UK) Chuan Wu (The University of Hong Kong, Hong Kong)

Fei Wu (The Pennsylvania State University, USA)

Jia $\mathrm{Wu}$ (Macquarie University, Australia)

Le Wu (Hefei University of Technology, China)

Liang Wu (Airbnb, Inc., USA)

Lingfei Wu (IBM Research AI, USA)

Sai Wu (Zhejiang University, China)

Shan-Hung Wu (National Tsing Hua University, Taiwan)

Yinghui Wu (Washington State University, USA)

Yulei Wu (UOE, UK)

Feng Xia (Dalian University of Technology, China)

Yinglong Xia (Futurewei Technologies, USA)

Lingyun Xiang (Changsha University of Science \& Technology, China)

Houping Xiao (Georgia State University, USA)

Keli Xiao (Stony Brook University, USA)

Yanghua Xiao (Fudan university, China)

Sihong Xie (Lehigh University, USA)

Sihong Xie (Lehigh University, USA)

Xiaohui Xie (Tsinghua University, China)

Yun Xiong (Fudan University, China)

Chang Xu (The University of Sydney, Australia)

Jian Xu (Alibaba Group, USA)

Jun Xu (School of Information, Renmin University of

China, China)
Zhao Xu (NEC Laboratories Europe, Germany)

Takehiro Yamamoto (University of Hyogo, Japan)

Rui Yan (Peking University, China)

Xiao Yan (LinkedIn, USA)

Bin Yang (Aalborg University, Denmark)

Bo Yang (Jilin University, China)

Hui Yang (san francisco state university, USA)

Qing Yang (University of North Texas, USA)

Yu Yang (City University of Hong Kong, Hong Kong)

Huaxiu Yao (The Pennsylvania State University, USA)

Lina Yao (The University of New South Wales, Australia)

Yibo Yao (Washington State University, USA)

Yuan Yao (Nanjing University, China)

Zijun Yao (IBM, USA)

Yanfang Ye (West Virginia University, USA)

Xu Yi (Beihang University, China)

Dawei Yin (JD.com, USA)

Hongzhi Yin (The University of Queensland, Australia)

Dantong Yu (New Jersey Institute of Technology, USA)

Guoxian Yu (Southwest University, China, China)

Hai-Tao Yu (University of Tsukuba, UK)

Linyun Yu (Bytedance Inc, China)

Ting Yu (Qatar Computing Research Institute, Qatar)

Yang Yu (Nanjing University, China)

Yanwei Yu (Yantai University, China)

Zhou Yu (Hangzhou Dianzi University, China)

Long Yuan (Nanjing University of Science \&

Technology, China)

Qiangqiang Yuan (wuhan university, China)

Ye Yuan (NEU, China)

Reza Zafarani (Syracuse University, USA)

Hamed Zamani (University of Massachusetts Amherst, USA)

Chengxi Zang (Tsinghua University \& Cornell University, USA)

Chunqiu Zeng (Google, USA)

Aidong Zhang (University of Virginia, USA)

Chao Zhang (Georgia Institute of Technology, USA)

Chao Zhang (Dalian University of Technology, China)

Chenwei Zhang (Amazon, USA)

Chuxu Zhang (University of Notre Dame, USA)

Dan Zhang (Facebook, USA)

Dongxiang Zhang (Zhejiang University, China)

Fan Zhang (Guangzhou University, China)

Jiawei Zhang (Florida State University, USA)

Junqi Zhang (Tsinghua University, China) 
Kun Zhang (Xavier University of Louisiana, USA)

Lefei Zhang (Wuhan University, China)

Min Zhang (Tsinghua University, China)

Qi Zhang (Fudan University, China)

Richong Zhang (Beihang University, China)

Shichao Zhang (Guangxi Normal University, China)

Shuhao Zhang (National University of Singapore, Singapore)

Si Zhang (University of Illinois Urbana-Champaign, USA)

Wei Zhang (East China Normal University, China)

Wei Emma Zhang (The University of Adelaide, Australia)

Weinan Zhang (Shanghai Jiao Tong University, China)

Weizhong Zhang (Zhejiang University, China)

Wenlu Zhang (California State University Long Beach, USA)

Xiang Zhang (The Pennsylvania State University, USA)

Xiangliang Zhang (King Abdullah University of Science \& Technology, Saudi Arabia)

Xiaoying Zhang (The Chinese University of Hong Kong, Hong Kong)

Xiuzhen Zhang (RMIT University, Australia)

Xuyun Zhang (The University of Auckland, New Zealand)

Ya Zhang (Shanghai Jiao Tong University, China)

Yanchun Zhang (Victoria University, Australia)

Yong Zhang (Huawei Technologies Canada Co., Ltd., Canada)

Zhao Zhang (Hefei University of Technology, China)

Zhenjie Zhang (LinkedIn, USA)

Zhiwei Zhang (Hong Kong Baptist University, Hong Kong)

Ziwei Zhang (Tsinghua University, China)

Haifeng Zhao (Linkedin, USA)

Peixiang Zhao (Florida State University, USA)

Shiwan Zhao (IBM Research - China, China)
Xiang Zhao (National University of Defense

Technology, China)

Xin Zhao (Renmin University of China, China)

Ying Zhao (Tsinghua University, China)

Yuhai Zhao (northeastern university, China)

Baihua Zheng (none, Singapore)

Kai Zheng (University of Electronic Science \&

Technology of China, China)

Weiguo Zheng (Fudan University, China)

Yudian Zheng (Twitter, USA)

Yukun Zheng (Tsinghua University, China)

Ning Zhong (Maebashi Institute of Technology, China)

Amelie Chi Zhou (Shenzhen University, China)

Aoying Zhou (East China Normal University, China)

Bin Zhou (University of Maryland, Baltimore County, USA)

Dawei Zhou (University of Illinois at Urbana-

Champaign, USA)

Fang Zhou (East China Normal University, China)

Xiangmin Zhou (RMIT University, Australia)

Xin Zhou (Nanyang Technological University,

Singapore)

Xun Zhou (University of Iowa, USA)

Yang Zhou (Auburn University, USA)

Yao Zhou (University of Illinois at Urbana-Champaign, USA)

Hengshu Zhu (Baidu Inc., China)

Lin Zhu (Linkedin, USA)

Xingquan Zhu (Florida Atlantic University, USA)

Yuanyuan Zhu (Wuhan University, China)

Fuzhen Zhuang (Institute of Computing Technology,

Chinese Academy of Sciences, China)

Yong Zhuang (University of Massachussets Boston, USA)

Zhongfang Zhuang (Visa Research, USA)

Imed Zitouni (Microsoft, USA)

Justin Zobel (The University of Melbourne, Australia)

Jia Zou (Arizona State University, USA) 


\section{CIKM'19 Program Committee Members - Applied Track}

Elliot Ai (Hangzhou Huawei Enterprises

Telecommunication Technologies Co., Ltd, China)

Jesse Anderton (Spotify, USA)

Hosein Azarbonyad (University of Amsterdam, Netherlands)

Ivan Brugere (Salesforce Research, USA)

Yingyi Bu (Google, USA)

Qian Chen (Google, China)

Rui Chen (Samsung Electronics, USA)

Yang Chen (LinkedIn, USA)

Michael Cole (LexisNexis, USA)

Bolin Ding (Alibaba Group, USA)

Bo Dong (Xi'an Jiaotong University, China)

Jinyang Gao (Alibaba, China)

Jiong He (Alibaba Group, Singapore)

Yeye He (Microsoft, USA)

Liangjie Hong (Etsy Inc., USA)

Chu-Cheng Hsieh (Amazon, USA)

Chao Huang (University of Notre Dame, USA)

Xiao Huang (Texas A\&M University, USA)

Xuanjing Huang (Fudan University, China)

Feng Ji (DAMO Academy, Alibaba Group, China)

Xianghu Ji (Huawei Technologies Co., Ltd, China)

Wenhao Jiang (Tencent, China)

Bo Jin (Dalian University of Technology, China)

Di Jin (Tianjin University, China)

Alekh Jindal (Microsoft, USA)

Deguang Kong (Google, USA)

Kun Kuang (Tsinghua University, China)

Jundong Li (University of Virginia, USA)

Yu-San Lin (Visa Research, USA)

Bin Liu (IBM TJ Watson Research Center, USA)

Guanfeng Liu (Macquarie University, Australia)

Qi Liu (University of Science \& Technology of China, China)

Yanchi Liu (Rutgers University, USA)

Yi Lu (Massachusetts Institute of Technology, USA)

Guixiang Ma (Univ, USA)

Jianxin Ma (Tsinghua University, China)

Xiaoxi Mao (Fuxi AI Lab, Netease Inc., China)

Umar Farooq Minhas (Microsoft, USA)

Abhishek Mukherji (Cisco Systems, USA)

Raghavan Muthuregunathan (Linkedin, USA)

Zahra Nazari (Spotify, USA)
Cagri Ozcaglar (LinkedIn, USA)

Xiao Qin (IBM, USA)

Minghui Qiu (Alibaba, Singapore)

Huiming Qu (IBM, USA)

Li Shen (Tencent AI Lab, China)

Wei Shen (Nankai University, China)

Yanyan Shen (Shanghai Jiao Tong University, China)

Guojie Song (Peking University, Beijing, China)

Jiliang Tang (Michigan State University, USA)

$\mathrm{Ke} \mathrm{Tu}$ (Tsinghua University, China)

Daixin Wang (Antfin Financial Services Group, China)

Jingyuan Wang (Beihang University, China)

Lanjun Wang (Huawei Technologies, China)

Xiao Wang (Beijing University of Posts and

Telecommunications, China)

Hao Wei (Amazon, USA)

Le Wu (Hefei University of Technology, China)

Liang Wu (Airbnb, Inc., USA)

Runze Wu (NetEase Fuxi AI Lab, China)

Yinglong Xia (Futurewei Technologies, USA)

Keli Xiao (Stony Brook University, USA)

Lin Yan (ByteDance Inc., China)

Yuan Yao (Nanjing University, China)

Zijun Yao (IBM, USA)

Kui Yu (Hefei University of Technology, China)

Long Yuan (Nanjing University of Science \&

Technology, China)

Ye Yuan (NEU, China)

Chengxi Zang (Tsinghua University \& Cornell

University, USA)

Chenwei Zhang (Amazon, USA)

Chuxu Zhang (University of Notre Dame, USA)

Feng Zhang (Renmin University of China, China)

Qi Zhang (Fudan University, China)

Rui Zhang (The University of Melbourne, Australia)

Weinan Zhang (Shanghai Jiao Tong University,

China)

Weizhong Zhang (Zhejiang University, China)

Ziwei Zhang (Tsinghua University, China)

Xiang Zhao (National University of Defense

Technology, China)

Xin Zhao (Renmin University of China, China) 
Kai Zheng (University of Electronic Science \& Technology of China, China)

Yudian Zheng (Twitter, USA)

Yanhui Zhong (HUAWEI Technologies co., LTD., China)
Hengshu Zhu (Baidu Inc., China)

Yuanyuan Zhu (Wuhan University, China)

Zhongfang Zhuang (Visa Research, USA) 


\section{CIKM'19 Program Committee Members - Demo Track}

Ankit Agrawal (Iowa State University, USA)

Nikita Bhutani (University of Michigan, USA)

Jiang Bian (University of Florida, USA)

Nikos Bikakis (University of Ioannina, Greece)

Petko Bogdanov (University at Albany-SUNY, USA)

Ivan Brugere (Salesforce Research, USA)

Prithwish Chakraborty (IBM, USA)

Feng Chen (University at Albany - SUNY, USA)

Nilaksh Das (Georgia Institute of Technology, USA)

Tyler Derr (Michigan State University, USA)

Lifang He (University of Pennsylvania, USA)

Zhe He (Florida State University, USA)

Mark Heimann (University of Michigan, USA)

Joyce Ho (Emory University, USA)

Xiao Huang (Texas A\&M University, USA)

Di Jin (University of Michigan, USA)

Minsuk Kahng (Georgia Institute of Technology, USA)

Xiangnan Kong (Worcester Polytechnic Institute, USA)

Luis A. Leiva (Aalto University, Finland)

Robert Moskovitch (Ben-Gurion University, Israel)
George Papastefanatos (IMIS / RC "Athena”, Greece)

Ioakeim Perros (HEALTH[at]SCALE, USA)

Xiao Qin (Worcester Polytechnic Institute, USA)

Neil Shah (Snap Research, USA)

Chao Shang (University of Connecticut, USA)

Jie Song (University of Michigan, USA)

Chang Su (Weill Cornell Medicine, Cornell

University, USA)

Jaroslaw Szlichta (University of Ontario - Institute of Technology, Canada)

Fengyi Tang (Michigan State University, USA)

Vincent Tseng (National Chiao Tung University,

Taiwan)

Dimitrios Tsoumakos (Ionian University, Greece)

Thanasis Vergoulis (IMSI, “Athena” RC, Greece)

Hongyi Wen (Cornell University, USA)

Yujun Yan (University of Michigan, USA)

Chengxi Zang (Tsinghua University \& Cornell

University, USA)

Guanjie Zheng (The Pennsylvania State University, USA) 


\section{CIKM 2019 Sponsors \& Supporters}

Sponsors: Supporter:

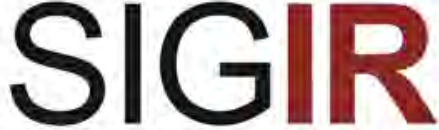

Special Interest Group on Information Retrieval

$$
\text { 优必选 }
$$

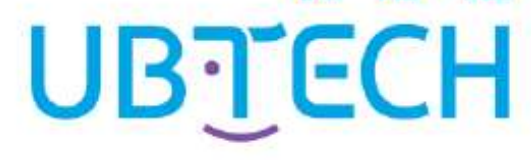

Gold Supporters:
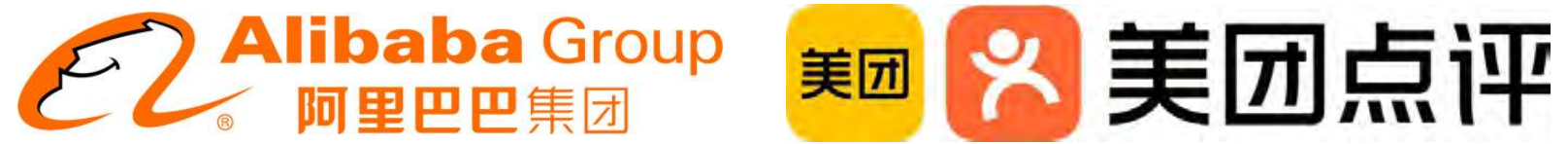

Silver Supporters:
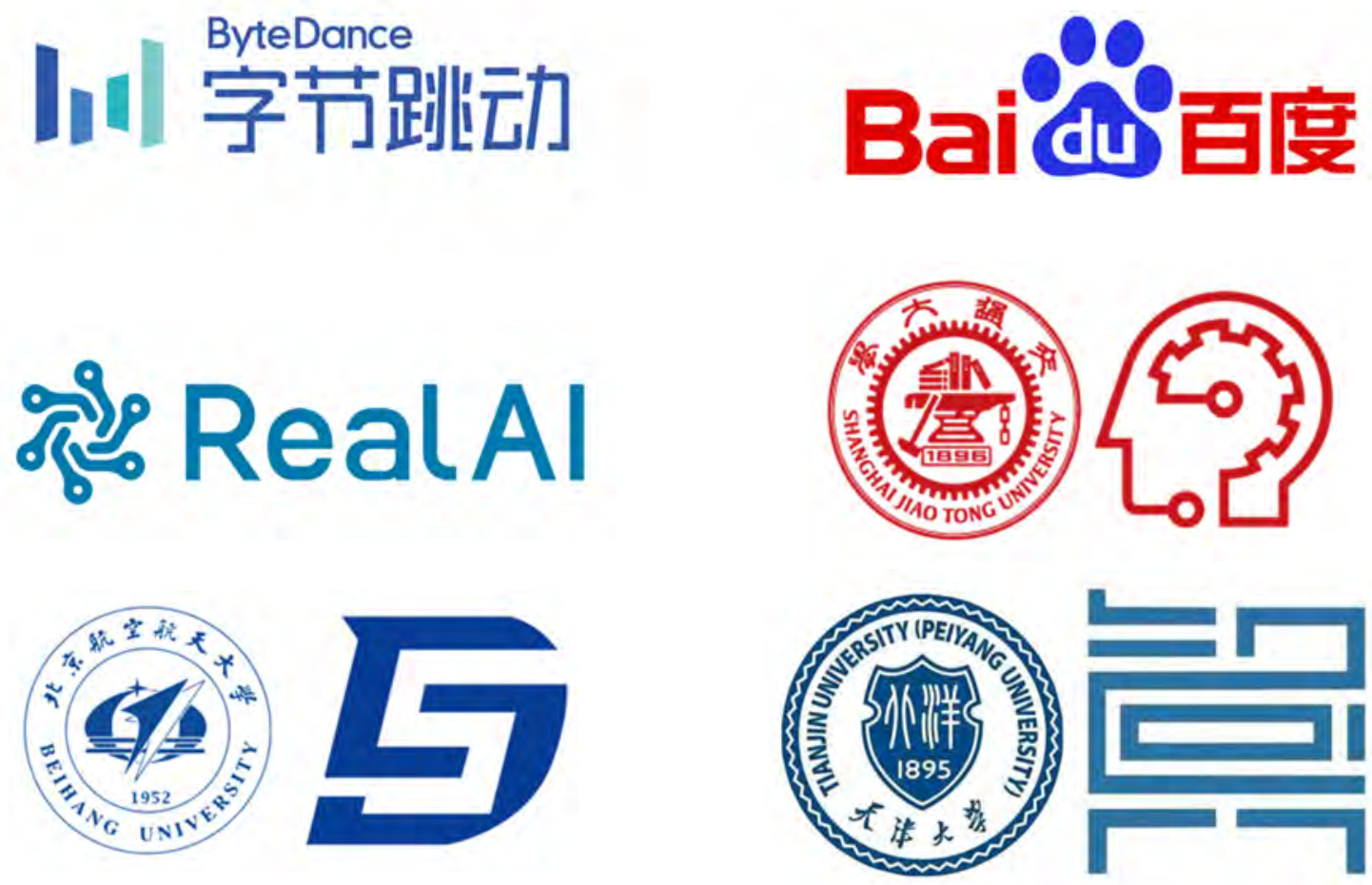

Bronze Supporter:

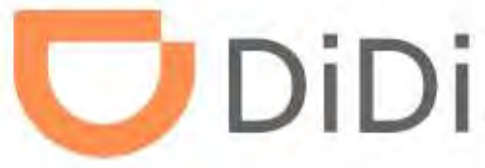

